\title{
Multiple-Photon Infrared Laser Photophysics and Photochemistry. II
}

\author{
V. N. BAGRATASHVILI, V. S. LETOKHOV, A. A. MAKAROV and E. A. \\ RYABOV \\ Institute of Spectroscopy, USSR Academy of Sciences, 142092, Troitsk, \\ Moscow Region, USSR
}

(Received June 15, 1982)

This is the second part of a review paper on the multiple-photon excitation of molecules by intense infrared laser light. This part commences with Section 3.

\section{Contents}

3. Elements of Theory of Resonant Interactions of IR Monochromatic Fields with Molecules ................ 3

3.1. Introduction to theoretical treatment $\ldots \ldots \ldots \ldots \ldots \ldots, 3$

3.1.1. Schrödinger equation and approximations $\ldots \ldots \ldots 3$

3.1.2. Quasienergetic or dressed quantum states ...... 5

3.2. Coherent excitation of various quantum systems $\ldots \ldots \ldots 6$

3.2.1. Two-level system $\ldots \ldots \ldots \ldots \ldots \ldots \ldots \ldots 6$

3.2.2. Harmonic oscillator ................ 13

3.2.3. Multiphoton transitions for multilevel system .... 17

3.2.4. Anharmonic oscillator ............... 25

3.3. Incoherent excitation of quantum system with relaxation $\ldots 28$

3.3.1. Conditions of incoherent excitation for two-level system .......................... 29

3.3.2. Effect of rotational bottleneck ........... 34 
3.4. Excitation from a discrete level into a quasicontinuous band ............................... 39

3.4.1. Derivation of the kinetic equations $\ldots \ldots \ldots \ldots, 40$

3.4.2. Validity of the kinetic equations $\ldots \ldots \ldots \ldots . \ldots 45$

3.5. Excitation of the vibrational quasicontinuum ........46

3.5.1. Kinetic equations for stimulated transitions in vibrational quasicontinuum .............47

3.5.2. Excitation for the case of constant cross-sections .. 51

3.5.3. Excitation for the case of varying cross-sections ...55

3.5.4. Influence of dissociative decay and rotational structure .....................58

3.5.5. Multiphoton transitions into quasicontinuum .....59

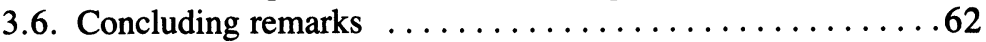

4. Physics of Interaction of a Polyatomic Molecule with an

Intense IR Field . .........................63

4.1. Main stages of MP excitation of polyatomic molecules

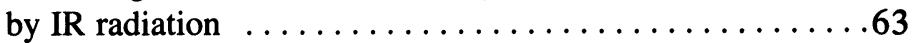

4.1.1. Role of IR radiation intensity in excitation of lower vibrational levels ................64

4.1.2. Role of IR radiation energy fluence in excitation of vibrational quasicontinuum $\ldots \ldots \ldots \ldots \ldots 66$

4.2. MP excitation of molecules at lower vibrational transitions ..........................669

4.2.1. "Nonsaturable" behavior of MP absorption of IR radiation by polyatomic molecules $\ldots \ldots \ldots \ldots 70$

4.2.2. Resonant character of MP absorption ........73

4.2.3. Fraction of MP excited molecules ..........76

4.3. MP excitation of molecules in the vibrational quasicontinuum ...................... 80

4.3.1. Evolution of MP absorption spectrum in the vibrational quasicontinuum ..............80

4.3.2. Distribution of vibrational energy of a molecule under MP excitation ..................85

4.4. Dissociation and MP excitation of molecules in continuum ........................90

4.4.1. "Threshold" character of dissociation yield .....90 90

4.4.2. Overexcitation of polyatomic molecules in continuum .....................95

4.5. MP excitation of the electronic states of polyatomic molecules by intense IR radiation 


\section{ELEMENTS OF THEORY OF RESONANT INTERACTIONS OF IR MONOCHROMATIC FIELDS WITH MOLECULES}

\subsection{Introduction to theoretical treatment}

The action of IR laser radiation on gases of polyatomic molecules comprises a substantial number of the fundamental questions dealing with the interaction of an isolated molecule with radiation calling for adequate theoretical treatment.

\subsubsection{Schrödinger equation and approximations}

The classical treatment of laser-radiation fields seems to be a common starting point for discussions with the following form of the operator of molecule-field interaction:

$$
\hat{V}(t)=-\hat{\mu} \mathbf{E}(t)
$$

quite substantiated theoretically (see, for example, Ref. 174) where $\hat{\boldsymbol{\mu}}$ is the dipole-moment operator of a molecule. The dependence of the electric component $\mathbf{E}$ of the field on spatial coordinates can be neglected, since the radiation wavelength is many orders larger than the molecular size. The validity of this description of laser radiation in terms of a monochromatic field is discussed in Section 2.8 from our previous paper, ${ }^{175}$ but as far as the theory is concerned, we shall follow in this series of papers just this approximation, since it makes the mathematics much easier. Of course, exceptions arising from radiation nonmonochromaticity will be qualitatively discussed when necessary. Thus, neglecting the spatial dependence of $\mathbf{E}$, let us take the electric field acting on the molecule as $\mathbf{E}=\mathscr{E}$ cos $\Omega t$ where the amplitude $\mathscr{E}$ is constant or, at least, slowly varying in time. Then the wavefunction of the molecule evolves in time in accordance with the Schrödinger equation

$$
i \hbar \frac{\partial \Psi}{\partial t}=\hat{H}_{0} \Psi-(\hat{\mu} \mathscr{E} \cos \Omega t) \Psi
$$

where $\hat{H}_{0}$ is the Hamiltonian of the molecule without an external field.

The description in terms of the wavefunction is absolutely equivalent to that in terms of the probability amplitudes referring to different quantum states of the stationary Hamiltonian $\hat{H}_{0}$ of the molecule. Indeed, if one presents the wavefunction $\Psi(t)$ as the expansion into series by stationary eigenfunctions $\psi_{j}$ or $\hat{H}_{0}$, i.e., 


$$
\Psi(t)=\sum_{j} a_{j}(t) \psi_{j} \exp \left(-\frac{i}{\hbar} E_{j} t\right)
$$

then $\Psi(t)$ can be fully determined through the temporal behavior of the probability amplitudes $a_{j}(t)$. It is easy to derive the equation for the amplitude $a_{k}(t)$ of the level $|k\rangle$. Substitute the expansion (3.3) into Eq. (3.2), multiply both sides by $\psi_{k}^{*} \exp \left(\frac{i}{\hbar} E_{k} t\right)$, integrate over the internal molecular coordinates, and use the orthogonality of stationary wavefunctions. Then one comes to

$$
\frac{d a_{k}}{d t}=i \sum_{j \neq k} \gamma_{k j} a_{j}\left\{\exp \left[i\left(\Omega-\omega_{j k}\right) t\right]+\exp \left[-i\left(\Omega+\omega_{j k}\right) t\right]\right\},
$$

where $\gamma_{k j}=\langle k|\boldsymbol{\mu}| j\rangle \mathscr{E} / 2 \hbar$. There are no terms with $j=k$ in sum, since all directions are, in particular, equivalent in nondegenerate states. Therefore, diagonal matrix elements of the dipole-moment operator vanish, and, for degenerate states, one can always choose the basis displaying the same property. Note also that the equality $\gamma_{k j}=\gamma_{j k}^{*}$ follows from the Hermiticity of the operator $\boldsymbol{\mu}$, and, since one may choose wavefunctions of stationary states to be real, then the equality $\gamma_{k j}=\gamma_{j k}$ can be taken without any additional reservations.

It can be seen from Eq. (3.4) that, even if their solutions may possibly be found, one can know in any case the frequencies and matrix elements of $\boldsymbol{\mu}$ for transitions between molecular levels. It is clear from the discussion of the vibration-rotation spectra of polyatomic molecules in Section 2 of our previous paper ${ }^{175}$ how hard the problems arising here can be. Nevertheless, just as theory gradually reveals the principal rules and features of the structure of molecular spectra starting from simple to more complex, the main features of appropriate spectroscopic models of excitation by monochromatic fields may be traced starting similarly from the simple. One should keep in mind one more simplification allowed in these processes due to the resonant character of excitation processes. In particular, it is possible as a good approximation to take into consideration not all transitions but rather only those which are close to resonance with the field. In this Section we shall work with Eq. (3.4) only for the simplest spectroscopic models. Some more complex models appropriate to more detailed treatments of vibration-rotation spectra will be discussed in the subsequent Sections, in close connection with experimental results. 
The topic of the interaction of radiation with an isolated molecule embraces nearly all the problems important for our goals. However, to understand some points, it is essential to take into account relaxation processes, and the simplest ideas will be discussed in Section 3.4. Other points related to the excitation of a gas as a whole (rather than an individual molecule) seem to be less essential. For our goals, the statistical distribution of molecules in the gas becomes only the averaging over initial conditions. But it should be noted that, in view of coherent transient effects (see, for example, Ref. 176) or nonlinear effects in gas media (see, for example, Ref. 177), the inclusion of a velocity distribution responsible for Doppler broadening becomes of fundamental importance. Also, we are not going to consider the influence of the medium on the radiation which can result in various propagation effects (see, for example, Refs. 178-180); i.e., a medium is believed to be optically thin in our theoretical treatment.

Finally, we will not take into account the spontaneous decay of excited molecular levels, since the typical radiative lifetime of about $10^{-1}-10^{-3} \mathrm{~s}$ in the IR range is several orders longer than the laser pulses used in experiments on IR excitation of molecules. We must stress, however, that if the lifetime becomes comparable with the pulse duration new qualitative effects will appear (see, for example, Ref. 181).

\subsubsection{Quasienergetic or dressed quantum states}

Before considering specific problems we wish to note rather a common feature of solutions of the nonstationary Schrödinger equation (3.2). This feature is that (see, for example, Ref. 182), if $\mathscr{E}=$ const, eigenfunctions of Eq. (3.2) may be presented in the following form:

$$
\Psi(t, q)=\varphi_{\varepsilon}(t, q) \exp (-i \varepsilon t),
$$

where $\varphi_{\varepsilon}$ are periodic functions of time, their cycle being just equal to $2 \pi / \Omega$. By their form, the eigenfunctions (3.5) resemble the conventional representation of stationary wavefunctions, and so the eigenvalues $\varepsilon$ are called quasienergies, and eigenstates (3.5) are called quasienergetic or, more commonly, dressed states. Since multiplying the function $\varphi_{\varepsilon}$ by the factor $\exp (i n \Omega t)$, where $n$ is an arbitrary integer, does not affect the property of periodicity, then one may conclude that quasienergies are defined with the accuracy to an arbitrary term $n \Omega$. The quasienergy approach is very useful, since some features of dressed states are very similar to those of ordinary stationary levels. In particular, resonances of spontaneous emis- 
sion as well as resonances of absorption and Raman scattering in second weak probe field are attached now to quasienergies. ${ }^{182}$

Another important fact (see Ref. 182) can be discovered for the case when the amplitude $\mathscr{E}$ of external field changes very slowly (adiabatically) from zero to some finite value. If initially (before the field is switched on) the system is in a definite stationary state $|i\rangle$, then at every subsequent instant of time the system is in that dressed state which adiabatically originates from the primary one, i.e., comes to $|i\rangle$ when $\mathscr{E} \rightarrow 0$. Of course, during this process the quasienergy itself as well as the dressed-state wavefunction will depend parametrically on the current amplitude of external field.

If, on the contrary, we are interested in another situation when the field amplitude is a step function being switched on at $t=0$, then, in the general case, the dependence of the wavefunction on time is described by the superposition of dressed-state eigenfunctions:

$$
\Psi(t)=\sum_{k} c_{k} \varphi_{\varepsilon_{\mathrm{k}}} \exp \left(-i \varepsilon_{k} t\right),
$$

where the factors $c_{k}$ must satisfy the initial condition at $t=0$.

\subsection{Coherent excitation of various quantum systems}

Let us start from the consideration of various simple quantum systems which are simplified models of a molecular system.

\subsubsection{Two-level system}

The first natural step is the consideration of a two-level system to obtain the simplest estimates for the excitation efficiency of the quantum system. In this case, the general equation (3.4) which describes the dynamics of probability amplitudes $a_{0}$ and $a_{1}$ of the lower and upper levels, correspondingly, take the following form:

$$
\begin{aligned}
& \frac{d a_{0}}{d t}=i \gamma_{01} a_{1}\left\{\exp \left[i\left(\Omega-\omega_{10}\right) t\right]+\exp \left[-i\left(\Omega+\omega_{10}\right) t\right]\right\} \\
& \frac{d a_{1}}{d t}=i \gamma_{01} a_{0}\left\{\exp \left[-i\left(\Omega-\omega_{10}\right) t\right]+\exp \left[i\left(\Omega+\omega_{10} t\right]\right\}\right.
\end{aligned}
$$


We are interested, of course, in the situation when the radiation frequency $\Omega$ is close to that $\omega_{10}$ of transition, i.e., at least the condition

$$
\left|\Omega-\omega_{10}\right| \ll \Omega
$$

is met. If so, then one can easily see that the exponential terms in the right-hand sides of Eqs. (3.7) differ greatly in frequency. To estimate relative contributions from these terms, let us first assume that perturbation theory can be used, i.e., with $a_{0}(t=0)=1$ and $a_{1}(t=0)=0$, at every subsequent instant of time $\left|a_{1}\right| \ll 1$. Accepting $a_{0} \equiv 1$ in the second of Eqs. (3.7) and integrating, we get

$$
\begin{aligned}
a_{1} & =\frac{\gamma_{01}}{\omega_{10}-\Omega}\left\{\exp \left[-i\left(\Omega-\omega_{10}\right) t\right]-1\right\} \\
& +\frac{\gamma_{01}}{\omega_{10}+\Omega}\left\{\exp \left[i\left(\Omega+\omega_{10}\right) t\right]-1\right\}
\end{aligned}
$$

It can easily be seen that, if condition (3.8) is fulfilled, the second term is much smaller than the first one. Therefore, one has to expect that the effect of an excitation of a two-level system arises mainly from the exponential term with the difference frequency.

The approximation in which the exponential terms with frequency $\Omega+$ $\omega_{10}$ are removed from Eqs. (3.7) is usually referred to as the rotatingwave approximation.* Besides the requirement (3.8), the second criterion for using the rotating-wave approximation is the condition

$$
\frac{\left|\mu_{01} \mathscr{E}\right|}{\hbar \omega_{10}} \ll 1
$$

For the transitions in the IR range, this condition is valid up to very strong radiation fields, the intensities of which are many orders higher than those ordinarily used in experiments. For example, taking the transition frequency $1000 \mathrm{~cm}^{-1}$ and the typical value $\mu_{01}=0.3$ Debye of dipole moment for some allowed vibration-rotation transition, one can find that the equality

*This name came from the visual vector model for the process of excitation of a two-level system introduced into theory in Ref. 183 by analogy with the nuclear magnetic resonance. 
$\mu_{01} \mathscr{E} / \hbar \omega_{10}=1$ requires an enormous intensity of radiation $I=c \mathscr{E}^{2} / 8 \pi$ $=2 \cdot 10^{14} \mathrm{~W} / \mathrm{cm}^{2}$.

We shall adhere below to the rotating-wave approximation without any additional reservation. In this approximation, Eqs. (3.7) take the form

$$
\begin{aligned}
& \left.\frac{d a_{0}}{d t}=i \gamma_{01} a_{1} \exp \left[i\left(\Omega-\omega_{10}\right) t\right)\right] \\
& \frac{d a_{1}}{d t}=i \gamma_{01} a_{0} \exp \left[-i\left(\Omega-\omega_{10}\right) t\right]
\end{aligned}
$$

the analytical solution of which is quite simple. Two independent solutions of Eqs. (3.11) may be presented, for example, as

$$
\left\|a_{0}^{(0)}\right\|=\left\|\begin{array}{l}
\left(\frac{\beta+1}{2 \beta}\right)^{1 / 2} \\
a_{1}^{(0)}
\end{array}\right\|\left(\frac{\beta-1}{2 \beta}\right)^{1 / 2} \operatorname{sign}\left(\frac{\Omega-\omega_{10}}{\gamma_{01}}\right) e^{-i\left(\Omega-\omega_{10) t}\right.} \| \exp \left[-i \frac{\Omega-\omega_{10}}{2}(\beta-1) t\right]
$$

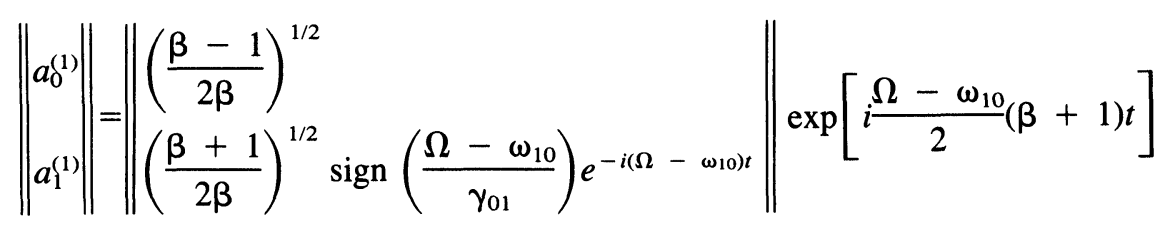

where $\beta=\left[1+4 \gamma_{01}^{2} /\left(\Omega-\omega_{10}\right)^{2}\right]^{1 / 2}$. Through direct substitution, one can make sure that these expressions really give solutions of Eqs. (3.11). Of course, the written-out solutions (3.12) and (3.13) comply with the normalization requirement $\left|a_{0}(t)\right|^{2}+\left|a_{1}(t)\right|^{2}=1$. By taking the superpositions of (3.12) and (3.13) one can combine any other solutions. But it is just each of solutions (3.12) and (3.13) that is eigen in the sense that it corresponds to a definite value of quasienergy. 
Indeed, the time-dependent wavefunction of two-level system has the form

$$
\begin{array}{r}
\Psi(t)=\left[\left(\frac{\beta+1}{2 \beta}\right)^{1 / 2} \psi_{0}\right. \\
\left.-\left(\frac{\beta-1}{2 \beta}\right)^{1 / 2} \operatorname{sign}\left(\frac{\Omega-\omega_{10}}{\gamma_{01}}\right) e^{-i \Omega t} \psi_{1}\right] \\
\exp \left\{-i\left[\frac{E_{0}}{\hbar}+\frac{\Omega-\omega_{10}}{2}(\beta-1)\right] t\right\}
\end{array}
$$

for the solution (3.12), and

$$
\begin{array}{r}
\Psi(t)=\left[\left(\frac{\beta-1}{2 \beta}\right)^{1 / 2} \psi_{0}\right. \\
\left.+\left(\frac{\beta+1}{2 \beta}\right)^{1 / 2} \operatorname{sign}\left(\frac{\Omega-\omega_{10}}{\gamma_{01}}\right) e^{-i \Omega t} \psi_{1}\right] \\
\exp \left\{-i\left[\frac{E_{0}}{\hbar}-\frac{\Omega-\omega_{10}}{2}(\beta+1)\right] t\right\}
\end{array}
$$

for the solution (3.13). The comparison with the general form (3.5) of dressed states convinces us that the first of our solutions (3.12) corresponds to the quasienergy

$$
\varepsilon_{0}=\frac{\Omega-\omega_{10}}{2}\left\{\left[1+\frac{4 \gamma_{01}^{2}}{\left(\Omega-\omega_{10}\right)^{2}}\right]^{1 / 2}-1\right\},
$$

and the second one to the quasienergy

$$
\varepsilon_{1}=-\frac{\Omega-\omega_{10}}{2}\left\{\left[1+\frac{4 \gamma_{01}^{2}}{\left(\Omega-\omega_{10}\right)^{2}}\right]^{1 / 2}+1\right\}
$$

if they are counted off the energy $E_{0}$ of the lower level. 
$a$

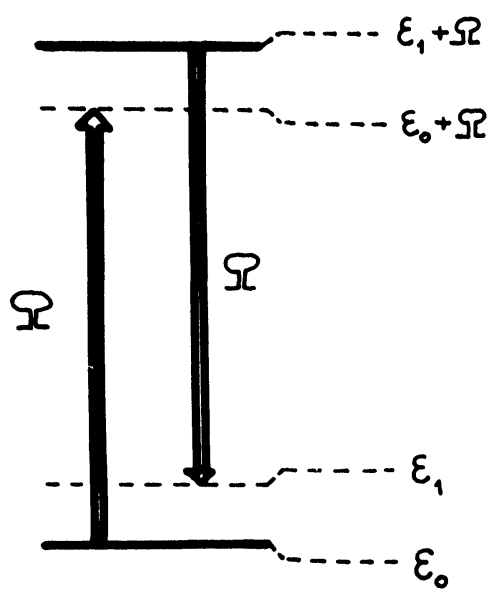

\section{b}

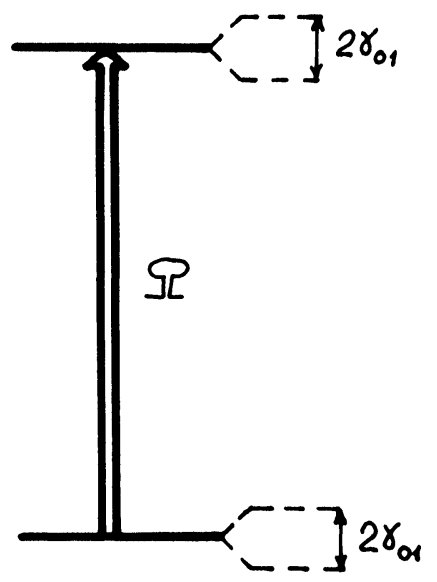

FIGURE 3.1 The diagram of dressed states when a near-resonant field is applied to a twolevel system. (a) Shift of levels due to the dynamic Stark effect. (b) Splitting of levels by exactly resonant field.

The diagram of dressed states is shown in Figure 3.1. If the field frequency is not equal exactly to the transition frequency, and the field amplitude tends to zero, then $\beta \rightarrow 1$, the dressed state (3.14) comes to the lower state, and the dressed state (3.15) comes to the upper state. The shift of levels in the presence of field is usually referred to as the shift due to the dynamic Stark effect. The case when the field frequency is exactly equal to the transition frequency is called degenerate. In this case, the quasienergies are just equal to $\varepsilon_{0,1}= \pm \gamma_{01}$; i.e., each of the dressed states cannot be attributed to any of two stationary levels. Therefore, one may often hear that the exactly resonant field leads to a splitting of levels.

Now let us trace the dynamics of excitation of a two-level system assuming that initially the system is in the lower state, and the field intensity is the step function being switched on at $t=0$. To do it one should [see the discussion on Eq. (3.6)] make up a superposition of solutions (3.12) and (3.13) which gives $a_{0}=1$ and $a_{1}=0$ at $t=0$. As a result, we get the following dependencies of probability amplitudes on time: 


$$
\begin{aligned}
a_{0}(t) & =\frac{\beta+1}{2 \beta} e^{-i \varepsilon_{0} t}+\frac{\beta-1}{2 \beta} e^{-i \varepsilon_{1} t} \\
& =\left[\cos \left(\frac{\Omega-\omega_{10}}{2} \beta \mathrm{t}\right)-\frac{i}{\beta} \sin \left(\frac{\Omega-\omega_{10}}{2} \beta t\right)\right] \exp \left(i \frac{\Omega-\omega_{10}}{2} t\right) \\
a_{1}(t) & =\frac{\left(\beta^{2}-1\right)^{1 / 2}}{2 \beta} \operatorname{sign}\left(\frac{\Omega-\omega_{10}}{\gamma_{01}}\right) e^{-i\left(\mathrm{i} \Omega-\omega_{10) t}\left(e^{-i \varepsilon_{1} t}-e^{-i \varepsilon_{0} t}\right)\right.} \\
& =\mathrm{i} \frac{\left(\beta^{2}-1\right)^{1 / 2}}{\beta} \operatorname{sign}\left(\frac{\Omega-\omega_{10}}{\gamma_{01}}\right) \sin \left(\frac{\Omega-\omega_{10}}{2} \beta t\right) \exp \left(-i \frac{\Omega-\omega_{10}}{2} t\right)
\end{aligned}
$$

and, for the probabilities of finding the system at the lower and upper levels (or, in other words, for the relative populations of levels), we have

$$
\begin{aligned}
\rho_{00}(t) & =a_{0}^{*}(t) a_{0}(t)=\cos ^{2}\left\{\frac{1}{2}\left[\left(\Omega-\omega_{10}\right)^{2}+4 \gamma_{01}^{2}\right]^{1 / 2} t\right\} \\
& +\frac{\left(\Omega-\omega_{10}\right)^{2}}{\left(\Omega-\omega_{10}\right)^{2}+4 \gamma_{01}^{2}} \sin ^{2}\left\{\frac{1}{2}\left[\left(\Omega-\omega_{10}\right)^{2}+4 \gamma_{01}^{2}\right]^{1 / 2} t\right\} \\
\rho_{11}(t) & =a_{1}^{*}(t) a_{1}(t)=\frac{4 \gamma_{01}^{2}}{\left(\Omega-\omega_{10}\right)^{2}+4 \gamma_{01}^{2}} \sin ^{2}\left\{\frac{1}{2}\left[\left(\Omega-\omega_{10}\right)^{2}+4 \gamma_{01}^{2}\right]^{1 / 2} t\right\}
\end{aligned}
$$

It may be seen from Eqs. (3.19) that the populations oscillate about their averaged values

$$
\begin{aligned}
& \overline{\rho_{00}(t)}=1-\frac{2 \gamma_{01}^{2}}{\left(\Omega-\omega_{10}\right)^{2}+4 \gamma_{01}^{2}}, \\
& \overline{\rho_{11}(t)}=\frac{2 \gamma_{01}^{2}}{\left(\Omega-\omega_{10}\right)^{2}+4 \gamma_{01}^{2}}
\end{aligned}
$$

In the case of exact resonance, i.e., with $\Omega=\omega_{10}$, and averaged values of relative populations of both levels are just equal to $\frac{1}{2}$, and the frequency of oscillation of probability amplitudes, the so-called Rabi frequency, is $\left|\gamma_{01}\right|$. With the detuning from exact resonance, it may be seen that the condition

$$
2\left|\gamma_{01}\right| \geq\left|\Omega-\omega_{10}\right|
$$


serves as the criterion of effective excitation, or, in other words, the halfwidth of the resonance at the half-maximum is equal to $2\left|\gamma_{01}\right|$. Therefore, the value $2\left|\gamma_{01}\right|$ is also called the power broadening.

Let us turn now to estimates. Take, for example, the intensity of the radiation field to be $10^{7} \mathrm{~W} / \mathrm{cm}^{2}$, and the transition dipole moment to be 0.3 Debye. Then the Rabi frequency $\left|\gamma_{01}\right|$ is $4.1 \times 10^{10} \mathrm{~s}^{-1}$. This means that in the case of exact resonance the time interval of the first semicycle of oscillations required for full inversion of populations is just $\pi / 2\left|\gamma_{01}\right|$ $=3.8 \times 10^{-11} \mathrm{~s}$. At the same time, with moving away exact resonance, the characteristic detuning $\left|\Omega-\omega_{10}\right|=2\left|\gamma_{01}\right|$ (which still allows us to treat the excitation of upper level as rather effective) is $0.44 \mathrm{~cm}^{-1}$. Now it is clear (see the discussion in Section 2.2.1 from our previous paper ${ }^{175}$ ) why, in view of the ordinary absorption spectroscopy which examines the resonances, one should use weak fields. Really, in the general case, even though the source is monochromatic, the spectral resolution drops as its intensity increases.

Thus, we have considered the dynamics of excitation of a two-level system at instantaneous switching on of the radiation field with the constant amplitude. In case of the exact resonance, the obtained expressions remain valid for fields with time-dependent amplitude, but only with the substitution.

$$
\mathscr{E} t \rightarrow \int_{-\infty}^{t} \mathscr{E}(\tau) d \tau
$$

In the general case of detuning from exact resonance, it is impossible to obtain analytical solutions. However, in the limit of adiabatically slow switching on of the field, according to the general principles [see the discussion following Eq. (3.5)], the state of the system being initially at the lower level will be described at all further instants by the solution (3.12) which corresponds to the quasienergy (3.16), the current value of field amplitude being put on. The criterion of adiabaticity here is the condition

$$
\left|\Omega-\omega_{10}\right| \tau_{\mathrm{sw}} \gg 1,
$$

where $\tau_{\mathrm{sw}}$ is the characteristic switching on time of the field. The criterion of excitation efficiency (3.21) remains valid for this case though the resonance half-width is somewhat smaller and comes to $2\left|\gamma_{01}\right| / \sqrt{3}$. 
Let us turn now to a discussion of the possible effects of nonmonochromaticity of radiation. It is hard, of course, to speak here about population oscillations, since they arise from the coherent nature of excitation. As far as the efficiency of upper-level excitation is concerned, in the case of detuning from the exact resonance which exceeds the width of the radiation spectrum, the estimates obtained remain valid, but one should use the modified Rabi frequency $\gamma_{01}^{\bmod }=\left(\mu_{01} / \hbar\right)(2 \pi I / c)^{1 / 2}$, where $I$ means the total field intensity integrated over its spectrum. When the field spectrum overlaps the transition frequency, the resonant part of the spectrum may be mainly responsible for excitation. Therefore, to estimate the time required for excitation one should take the intensity of that part of the field spectrum which lies within the value of power broadening. Thus, if the power broadening exceeds the width of the field spectrum, then one may use the above formulas to estimate the excitation time. Actually this calls for rather moderate intensities of radiation. For example, with a transition dipole moment of 0.3 Debye and spectrum width of $(\Delta \Omega)_{\mathrm{rad}}=0.03 \mathrm{~cm}^{-1}$, the equality $2\left|\gamma_{01}\right|=(\Delta \Omega)_{\text {rad }}$ is reached at the intensity $4.6 \times 10^{4} \mathrm{~W} / \mathrm{cm}^{2}$. One more point should be noted. If the modes of radiation of laser are synchronized (see Section 2.8 from Ref. 175), the intensity at some instant may essentially exceed its mean value. This should be taken into account in estimates.

\subsubsection{Harmonic oscillator}

The harmonic oscillator is the simplest approximation for the levels of an individual vibrational mode of a molecule. In view of behaviors in external nonstationary fields, the harmonic oscillator is also the simplest case, since it is possible to obtain analytical solutions of the Schrödinger equation for an arbitrary dependence of the field amplitude on time (see, for example, Ref. 184). We shall consider here only the solution for the case of a monochromatic field and, for simplicity, adhere to the rotating-wave approximation. The exact solution, which is much more cumbersome, gives only small corrections. So, let us consider Eq. (3.4) for the probability amplitudes of an infinite set of levels of the harmonic oscillator. Since, within the harmonic approximation, only transitions between adjacent levels are allowed, we get the set of equations

$$
\begin{aligned}
\frac{d a_{v}}{d t}=i \gamma\left\{v^{1 / 2} a_{v-1} \exp [\right. & -i(\Omega-\omega) t] \\
& +(v+1)^{1 / 2} a_{v+1} \exp [i(\Omega-\omega) t]
\end{aligned}
$$


where $\gamma=\gamma_{01}$, the dependence of the dipole-moment matrix element on the number of transition is taken into account [see Eq. (2.44) from Ref. 175], and only the terms with the difference frequency are retained. Since all transition frequencies between adjacent levels of the harmonic oscillator are equal, then frequency indexes are omitted in Eq. (3.24).

First, we shall consider the case when the oscillator frequency is not exactly equal to that of the field, i.e., $\Omega \neq \omega$. Let us transform Eq. (3.24) using the substitution $\tilde{a}_{v}=a_{v} \exp [i v(\Omega-\omega) t]$. For the new probability amplitudes $\tilde{a}_{v}$ which differ from $a_{v}$ by exponential phase factors one has the following set of equations with constant coefficients:

$$
\frac{d \tilde{a}_{v}}{d t}-i v(\Omega-\omega) \tilde{a}_{v}=i \gamma\left[v^{1 / 2} \tilde{a}_{v-1}+(v+1)^{1 / 2} \tilde{a}_{v+1}\right]
$$

According to the general theory of linear differential equations, solutions of (3.25) may be written in the form

$$
\tilde{a}_{v}=\alpha_{v} \exp (-i \varepsilon t)
$$

Now for $\alpha_{v}$ we have the set of equations

$$
-[\varepsilon+v(\Omega-\omega)] \alpha_{v}=\gamma\left[v^{1 / 2} \alpha_{v-1}+(v+1)^{1 / 2} \alpha_{v+1}\right],
$$

and it can be seen that eigenvalues of $\varepsilon$, i.e., such values which are consistent with nontrivial solutions of (3.27), are just the quasienergies. However, at first sight it is not clear yet what is to be called eigenvalues in our case, since the set (3.27) is infinite. Indeed, with an arbitrary $\varepsilon$, setting, for example, $\alpha_{0}=$ const one can find $\alpha_{1}$ from the first equation, $\alpha_{2}$ from the second equation, etc. This procedure may go on to infinity. However, from normalization requirements it becomes obvious that one must choose only such solutions which, in any case, comply with the condition

$$
\lim _{v \rightarrow \infty} \alpha_{v}=0
$$

The values of $\varepsilon$, for which the condition (3.28) is met, will give just the quasienergy spectrum. Here one may see the full analogy with the way of finding the discrete eigenvalues of energy in stationary quantum systems, where the requirement is for the wavefunction to tend to zero at infinity. 
Thus, the common procedure to obtain solutions is rather clear. This does not mean yet that they can be written out explicitly in a closed form. Fortunately, in our specific case the chain of equations leads to rather simple expressions for $\alpha_{v}$ through the so-called Laguerre polynomials, the properties of which are well known:

$$
\alpha_{v}=\text { Const } \times\left(\frac{\Omega-\omega}{\gamma}\right)^{v}(v !)^{1 / 2} L_{v}^{y}-v\left[\left(\frac{\gamma}{\Omega-\omega}\right)^{2}\right],
$$

where $y=-\varepsilon /(\Omega-\omega)+\gamma^{2} /(\Omega-\omega)^{2}$. In the explicit form, the Laguerre polynomials are

$$
\begin{aligned}
L_{v}^{y}-v(x) & =\frac{(-x)^{v}}{v !}+\frac{y}{1 !} \frac{(-x)^{v-1}}{(v-1) !}+\frac{y(y-1)}{2 !} \frac{(-x)^{v-2}}{(v-2) !}+\ldots \\
& +\frac{y(y-1) \cdot \ldots \cdot(y-v+1)}{v !}
\end{aligned}
$$

and one can make sure through direct substitution that $\alpha_{v}$ (3.29) really satisfies Eq. (3.27). Furthermore, from the explicit form of Laguerre polynomials (3.30) it can be established that the requirement (3.28) is fulfilled if only the parameter $y$ is equal to zero or to any positive integer. Therefore, we obtain the quasienergy spectrum

$$
\varepsilon_{v}=-v(\Omega-\omega)+\frac{\gamma^{2}}{\Omega-\omega}
$$

The numeration in (3.31) has already been given in such a form that as the field amplitude (or the $\gamma$ value) tends to zero the $v$-th dressed state comes to the $v$-th energy level.

In particular, the dressed state with the quasienergy $\varepsilon_{0}=\gamma^{2} /(\Omega-\omega)$ is adiabatically connected with the ground state of the oscillator, and, according to the general principles, during adiabatically slow switching on of the field the initially nonexcited oscillator has to be just in this dressed state. In this case, the distribution of populations over various levels, the normalization in (3.29) being taken into account, is given by the following equation:

$$
\rho_{v v}=\frac{1}{v !}\left(\frac{\gamma}{\Omega-\omega}\right)^{2 v} \exp \left[-\left(\frac{\gamma}{\Omega-\omega}\right)^{2}\right]
$$


This is the well-known Poisson distribution with the mean energy

$$
\langle v\rangle=\left(\frac{\gamma}{\Omega-\omega}\right)^{2}
$$

expressed in terms of the energy of one oscillator quantum. So, one can conclude that effective excitation of the $v$-th level of the harmonic oscillator under slow switching on of the frequency-detuned monochromatic field requires that the ratio of the Rabi frequency $\gamma$ at the transition $0 \rightarrow 1$ to the value $|\Omega-\omega|$ of detuning should be no less than $v^{1 / 2}$. The criterion of adiabaticity in this case is the same as the condition (3.23) for a twolevel system.

In the case of stepwise switching on of the field, the dependence of wavefunction of oscillator, or, in other words, the dynamics of excitation is described by a superposition of dressed states. We omit here simple intermediate calculations and write out at once the result in terms of populations depending on time:

$$
\rho_{v v}(t)=\frac{\langle v(t)\rangle^{v}}{v !} \exp [-\langle v(t)\rangle]
$$

where the mean energy of the oscillator $\langle v(t)\rangle$ in one-quantum-energy units is

$$
\langle v(t)\rangle=\frac{4 \gamma^{2}}{(\Omega-\omega)^{2}} \sin ^{2}\left[\frac{1}{2}(\Omega-\omega) t\right]
$$

It should be noted that the expression for mean energy accurately coincides with the corresponding classical result. It follows from Eqs. (3.34) and (3.35) that the maximum of distribution oscillates in time with the frequency $|\Omega-\omega|$ between the ground state and the levels with $v \simeq v_{\max }$ $=4 \gamma^{2} /(\Omega-\omega)^{2}$.

Eq. (3.35) enables us to trace what occurs if $\Omega \rightarrow \omega$, i.e., in the exactly resonant case. As in the case of the classical oscillator in an exactly resonant external field, the mean energy grows without limit according to the law

$$
\langle v(t)\rangle=\gamma^{2} t^{2}
$$

Hence one can estimate, for example, that, with a dipole moment of the lower transition of 0.3 Debye, the harmonic oscillator is excited by field 
with intensity $10^{7} \mathrm{~W} / \mathrm{cm}^{2}$ to levels with $v=2 \times 10^{7}$ during the time of 100 ns, i.e., absorbs an enormous energy. The possibility of such strong excitation is caused only by the equidistant energy level spectrum of the harmonic oscillator. This feature is also typical for some other quantum systems with similarly equispaced energy levels in their spectra. ${ }^{185}$ The nonequidistanced levels of real spectra caused by anharmonicity for molecular vibrations bring about drastic changes in estimating the efficiency of excitation. This can be seen if one estimates, with the above parameters of oscillator and field, the value of detuning which leads to $v_{\max } \simeq 1$. Such an estimate gives rather a small value $|\Omega-\omega| \simeq 0.44 \mathrm{~cm}^{-1}$. Since the typical values of anharmonic shifts in molecular spectra are larger, it becomes clear to what extent the harmonic oscillator model is not adequate to the treatment of an individual vibrational mode in view of its interaction with radiation.

\subsubsection{Multiphoton transitions for multilevel system}

Three-level system. It is convenient to begin the examination of multiphoton resonant processes by discussing the dynamics of excitation of the three-level system shown in Figure 3.2. We consider the case when the frequencies of two successive transitions are not equal, i.e., $\omega_{10} \neq$ $\omega_{21}$. From estimates for the two-level system obtained in Section 3.1 one may expect that with, for example, the field frequency $\Omega$ coinciding with $\omega_{10}$, the excitation of the highest level is probably effective if the value $2\left|\gamma_{12}\right|$ of power broadening for the transition $1 \rightarrow 2$ is greater than or of the order of the corresponding detuning $\left|\Omega-\omega_{21}\right|=\left|\omega_{10}-\omega_{21}\right|$. In the opposite case when

$$
\gamma_{12} \ll\left|\omega_{10}-\omega_{21}\right|,
$$

the oscillations must be observed only between two lower levels and the upper level will be little populated. It is interesting, however, to examine what will be observed if the field frequency is taken to be

$$
\Omega=\frac{\omega_{10}+\omega_{21}}{2}
$$

chosen so that the excitation of the upper level from the lower one should be resonant in energy with respect to the process of absorption of two 


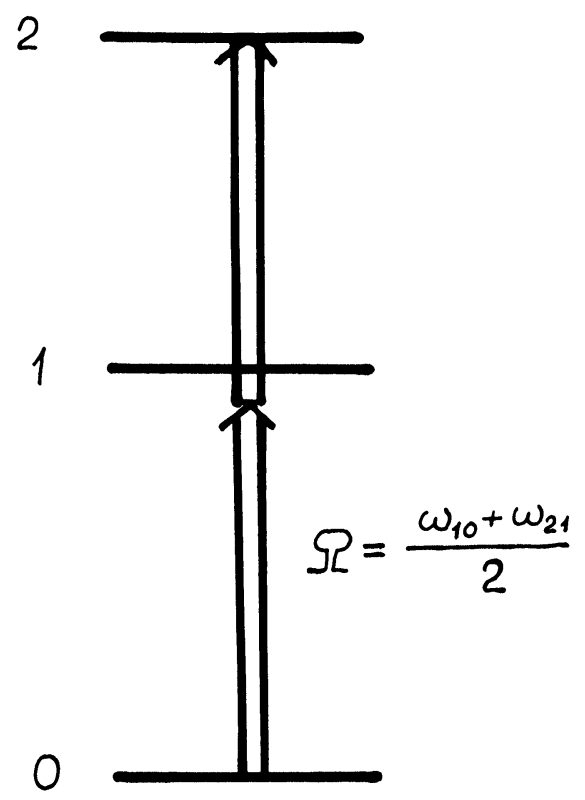

FIGURE 3.2 Two-photon resonance in a nonequidistant three-level system.

photons at once. Eq. (3.4) for probability amplitudes in this case has the following form:

$$
\begin{aligned}
& \frac{d a_{0}}{d t}=i \gamma_{01} a_{1} \exp \left(i \frac{\omega_{21}-\omega_{10}}{2} t\right) \\
& \frac{d a_{1}}{d t}=i \gamma_{01} a_{0} \exp \left(i \frac{\omega_{10}-\omega_{21}}{2} t\right)+i \gamma_{12} a_{2} \exp \left(i \frac{\omega_{10}-\omega_{21}}{2}\right) t \\
& \frac{d a_{2}}{d t}=i \gamma_{12} a_{1} \exp \left(i \frac{\omega_{21}-\omega_{10}}{2} t\right),
\end{aligned}
$$

the rotating-wave approximation being used.

Let us introduce here the designation $\delta=\left(\omega_{21}-\omega_{10}\right) / 2 \equiv \Omega-\omega_{10}$ and make in Eqs. (3.39) the substitution $\tilde{a}_{1}=a_{1} \exp (i \delta t)$. As a result, one gets equations with constant coefficients and after the substitution

$$
a_{0,2}=\alpha_{0,2} e^{-i \varepsilon t}, \tilde{a}_{1}=\alpha_{1} e^{-i \varepsilon t}
$$


gets the following set of linear equations for $\alpha_{k}$ :

$$
\begin{aligned}
& \varepsilon \alpha_{0}+\gamma_{01} \alpha_{1}=0 \\
& \gamma_{01} \alpha_{0}+(\varepsilon+\delta) \alpha_{1}+\gamma_{12} \alpha_{2}=0 \\
& \gamma_{12} \alpha_{1}+\varepsilon \alpha_{2}=0
\end{aligned}
$$

which is intended for finding the eigenvalues of quasienergy $\varepsilon_{i}(i=0,1$, 2) through the requirement that its determinant be equal to zero. This requirement leads to the cubic equation

$$
\varepsilon^{3}+\delta \varepsilon^{2}-\left(\gamma_{01}^{2}+\gamma_{12}^{2}\right) \varepsilon=0,
$$

the roots of which in our specific case are

$$
\varepsilon_{0}=0, \varepsilon_{1,2}=-\frac{\delta}{2}\left\{1 \pm\left[1+\frac{4\left(\gamma_{01}^{2}+\gamma_{12}^{2}\right)}{\delta^{2}}\right]^{1 / 2}\right\}
$$

Now we have everything to write out the solutions for probability amplitudes corresponding to each dressed state and make up their superposition which describes the dynamics of excitation of a system being initially at $t=0$ in the lower level. First, let us assume that $\gamma_{01}=\gamma_{12}=\gamma$ and use the condition (3.37). Neglecting the terms of the order of $\gamma / \delta$ one finds that the system oscillates between the lower and upper levels according to

$$
a_{0}(t) \simeq \exp \left(-i \frac{\gamma^{2}}{\delta} t\right) \cos \frac{\gamma^{2}}{\delta} t, a_{2}(t) \simeq-i \exp \left(-i \frac{\gamma^{2}}{\delta} t\right) \sin \frac{\gamma^{2}}{\delta} t
$$

whereas the probability amplitude of the intermediate level is small (about $\gamma / \delta)$. Hence we have for populations

$$
\rho_{00}(t) \simeq \cos ^{2} \frac{\gamma^{2}}{\delta} t, \rho_{22}(t) \simeq \sin ^{2} \frac{\gamma^{2}}{\delta} t
$$

By analogy with the two-level system, the value $\gamma^{2} /|\delta|$ is called the two-photon Rabi frequency $\gamma^{(2)}$, and further we have to discuss the way for the obtained expression to be generalized to the case when $\gamma_{01} \neq \gamma_{12}$, i.e., dipole moments of two transitions are not equal. One may assume two following simple formulas which generalize the result just obtained: 


$$
\gamma^{(2)}=\left|\frac{\gamma_{01} \gamma_{12}}{\delta}\right|
$$

and

$$
\gamma^{(2)}=\frac{\gamma_{01}^{2}+\gamma_{12}^{2}}{2|\delta|}
$$

The second equation (3.47) is formally true for the case of exact twophoton resonance (see the discussion in Ref. 185). Indeed, if with the use of quasienergy values (3.43) one writes out explicitly, for example, the dependence of the probability amplitude of the upper level on time, the following equation will be found:

$$
\rho_{22}(t) \simeq \frac{4 \gamma_{01}^{2} \gamma_{12}^{2}}{\left(\gamma_{01}^{2}+\gamma_{12}^{2}\right)^{2}} \sin ^{2} \frac{\gamma_{01}^{2}+\gamma_{12}^{2}}{2 \delta} t
$$

However, we may notice that the population of the upper level in this case does not reach unity at any instant. Moreover, if the dipole moments of two transitions differ greatly, then the population of the upper level should be much smaller than unity. The cause of this oscillation of populations is that at different dipole moments of two transitions the frequency of exact two-photon resonance is not optimum for the excitation of the upper level. Let us consider the transitions $0 \rightarrow 1$ and $1 \rightarrow 2$ separately (see Figure 3.3). As discussed in Section 3.1 (Figure 3.1), the monochromatic field detuned from the resonance is responsible for shifts of levels due to the dynamic Stark effect. It may be seen that the intermediate level, detunings being equal in value but different in sign (such detunings are at the exact two-photon resonance), and dipole moments of transitions being different, affects the shifts of lower and upper levels in different ways. Therefore, the frequency optimum for the two-photon transition effectively shifts. The shift of the frequency $\Omega_{\mathrm{opt}}$ away from $\left(\omega_{10}+\omega_{21}\right) /$ 2 is small, and, in our case when $\left|\gamma_{01}\right|,\left|\gamma_{12}\right| \ll|\delta|$, it will be

$$
\Omega_{\mathrm{opt}}-\frac{\omega_{10}+\omega_{21}}{2} \simeq \frac{\gamma_{12}^{2}-\gamma_{01}^{2}}{2 \delta}
$$

It is interesting to point out that, in the general case, this shift depends on the field amplitude. ${ }^{186}$ 


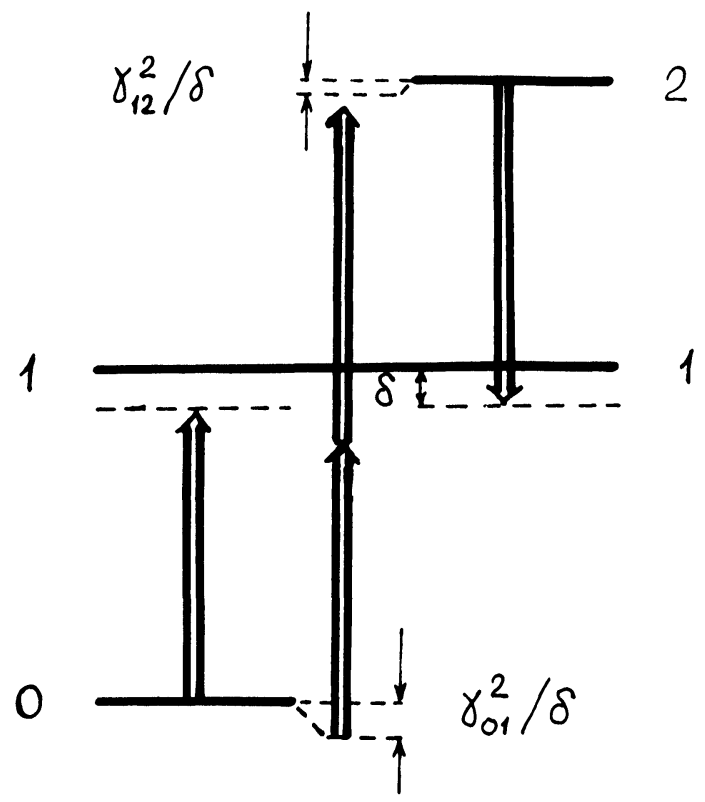

FIGURE 3.3 The effect of different shifts of levels due to the dynamic Stark effect on the value of frequency which is optimum for the two-photon transition.

More accurate analysis shows that, when the field at the frequency $\Omega_{\text {opt }}$ is applied, the system really oscillates between the lower and upper levels. As far as the value of two-photon Rabi frequency is concerned, one should now use Eq. (3.46) at the frequency $\Omega_{\mathrm{opt}}$. Thus, during the excitation at $\Omega_{\mathrm{opt}}$ the dynamics of populations of the lower and upper levels is described by the following equations:

$$
\rho_{00}(t) \simeq \cos ^{2} \frac{\gamma_{01} \gamma_{12}}{\delta} t, \quad \rho_{22}(t) \simeq \sin ^{2} \frac{\gamma_{01} \gamma_{12}}{\delta} t
$$

To modify more general equations (3.19) which involve the detuning from resonance to the case of a two-photon transition one should simply use $\gamma^{(2)}$ (3.46) instead of $\gamma$ and write the detuning in the form $2 \Omega-2 \Omega_{\text {opt }}$, since it really gives the difference between the energy of two photons divided by $\hbar$ and the frequency of transition. As a result, we get the following equations: 


$$
\begin{aligned}
\rho_{00}(t) & \simeq \cos ^{2}\left\{\left[\left(\Omega-\Omega_{\mathrm{opt}}\right)^{2}+\frac{\gamma_{01}^{2} \gamma_{12}^{2}}{\delta^{2}}\right]^{1 / 2} t\right\} \\
& +\frac{\left(\Omega-\Omega(\mathrm{opt})^{2}\right.}{\gamma_{01}^{2} \gamma_{12}^{2} / \delta^{2}+\left(\Omega-\Omega_{\mathrm{opt}}\right)^{2}} \sin ^{2}\left\{\left[\left(\Omega-\Omega_{\mathrm{opt}}\right)^{2}+\frac{\gamma_{01}^{2} \gamma_{12}^{2}}{\delta^{2}}\right]^{1 / 2} t\right\} \\
\rho_{22}(t) & \simeq \frac{\gamma_{01}^{2} \gamma_{12}^{2} / \delta^{2}}{\gamma_{01}^{2} \gamma_{12}^{2} / \delta^{2}+\left(\Omega-\Omega_{\mathrm{opt}}\right)^{2}} \sin ^{2}\left\{\left[\left(\Omega-\Omega_{\mathrm{opt}}\right)^{2}+\frac{\gamma_{01}^{2} \gamma_{12}^{2}}{\delta^{2}}\right]^{1 / 2} t\right\}
\end{aligned}
$$

which become Eqs. (3.50) when $\Omega=\Omega_{\text {opt. }}$. The half-width of the twophoton resonance, or in other words, the value of two-photon power broadening is just equal to $\gamma^{(2)}(3.46)$, i.e.,

$$
(\Delta \Omega)^{(2)}=\gamma^{(2)}=\left|\frac{\gamma_{01} \gamma_{12}}{\delta}\right|
$$

It should be noted that, comparing the second Eq. (3.51) with the exact solution (3.48) describing the excitation at the frequency $\Omega=\left(\omega_{10}+\right.$ $\left.\omega_{21}\right) / 2$, one may find them to just coincide when our estimate (3.49) is taken for the frequency $\Omega_{\mathrm{opt}}$.

$N$-level system. The generalization of obtained derivations for cases of resonances of greater multiplicity (three-photon, four-photon, etc.) looks rather simple. ${ }^{185}$ Let, for example, one intermediate level coupled with the lower and upper levels through allowed transitions fall on each quantum of radiation frequency in a one to one correspondence (see Figure 3.4) until the $N$-th level is in the $N$-photon resonance with the ground one, i.e., the frequency of field is approximately equal to $\left(\omega_{10}+\omega_{21}+\ldots+\right.$ $\left.\omega_{N, N-1}\right) / N$ with the correction arising from shifts due to the dynamic Stark effect. Then the multiphoton Rabi frequency is

$$
\gamma^{(N)}=
$$

$\left|\frac{\gamma_{01} \gamma_{12} \cdot \ldots \cdot \gamma_{N-1, N}}{\left(\Omega-\omega_{10}\right)\left(2 \Omega-\omega_{10}-\omega_{21}\right) \cdot \ldots \cdot\left[(N-1) \Omega-\omega_{10}-\omega_{21}-\ldots-\omega_{N-1, N-2}\right]}\right|$

For this expression to be valid, it is necessary, of course, that any intermediate resonance be absent, i.e., for all intermediate transitions, multiphoton Rabi frequencies should be much smaller than corresponding de- 


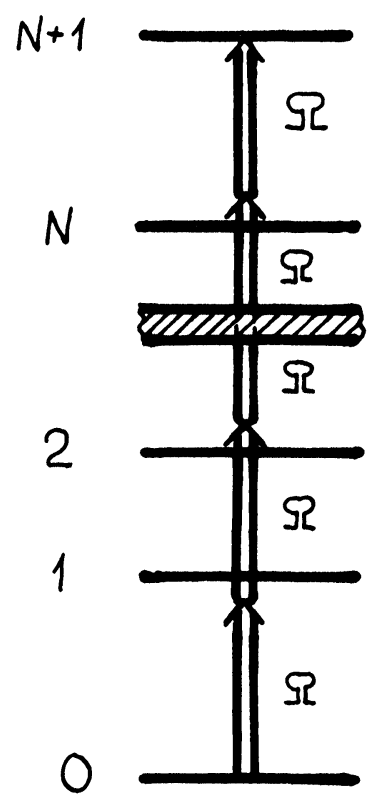

FIGURE 3.4 $N$-photon resonance in the quasiequidistant system with $(N+1)$ levels.

tunings. With this reservation, the dynamics of populations at multiphoton resonance is described by the equations

$$
\rho_{00}(t) \simeq \cos ^{2}\left[\gamma^{(N)} t\right], \quad \rho_{\mathrm{NN}}(t) \simeq \sin ^{2}\left[\gamma^{(N)} t\right],
$$

and the half-width of the multiphoton resonance is

$$
(\Delta \Omega)^{(N)}=2 \gamma^{(N)} / N
$$

Particular estimates for rates of multiphoton transitions will be given in the next section when the anharmonic oscillator will be considered, for which multiphoton resonances are of special importance. Here we only wish to discuss two more important points. The above formulas for multiphoton Rabi frequencies can be applied to the simplest case when one intermediate level falls on or corresponds to each quantum of field frequency. In the general case, there are perhaps several channels (see Figure 3.5a), and one should take into account their interference. Summing must 
a

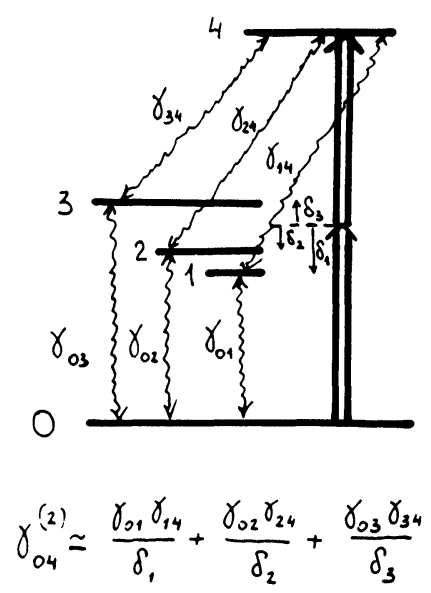

$b$

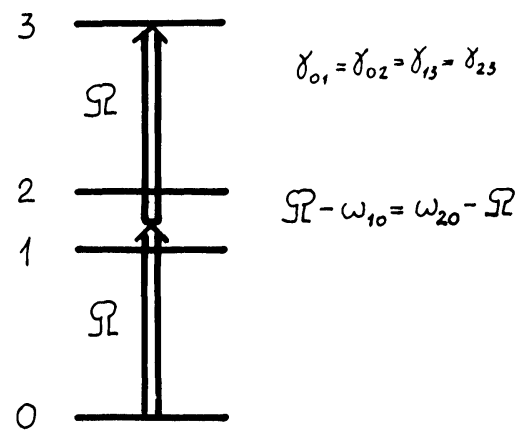

$\gamma_{03}^{(2)}=0$

FIGURE 3.5 (a) Interference of several channels at the multiphoton resonance. (b) The example of two-photon resonance with the Rabi frequency equal to zero because of the interference.

be carried out with the use of expressions like (3.53), but with some allowance made for the signs of transition dipole moments as well as detunings. Figure 3.5b shows a simple example of a two-photon transition with two intermediate levels with opposite detunings. The reader may practice solving the equations for probability amplitudes and make sure that, in this case, two-photon transitions do not really occur. The formal summation of two-photon Rabi frequencies with sign-opposite detunings at two intermediate levels also leads to zero.

Thus, the interference of various channels may cause the rate of multiphoton transition not to increase obviously but probably to decrease as compared to the rate calculated for any separate channel.

The second important point concerns the possible role of the nonmonochromaticity of radiation. Here the case is just as that for the two-level system. In particular, only the resonant part of field spectrum may play a role, and one must take into account this point when estimating rates of multiphoton transitions. It should be noted, however, that multiphoton Rabi frequencies are ordinarily much smaller than Rabi frequencies in the 
two-level case. Therefore, the part of the field spectrum which may be treated as resonant is much narrower. The characteristic estimates will be seen from the instances considered in the next section.

\subsubsection{Anharmonic oscillator}

To allow for anharmonicity in a separate vibrational mode is the next step after the harmonic approximation, when developing the theory of vibrational spectra. As noted in Ref. 175 (Section 2.1.3), a convenient approximation used here is the Morse oscillator, the energy levels of which are given by Eq. (2.23) from Ref. 175. We do not write out the equations of motion for probability amplitudes in this case, since these equations cannot be solved analytically. However, we already know enough to understand the basic feature regularities of the dynamics of the anharmonic oscillator depending on the frequency and amplitude of the exciting field. If the frequency of field coincides with that of the transition $v=0 \rightarrow v$ $=1$ of oscillator, and the condition (3.37) is fulfilled, then population oscillations between principally the pair of lower levels will occur, and the excitation of even the next level $v=2$ will be small. Otherwise, if the field frequency is shifted to the long-wave region, then successive multiphoton resonances will be observed at the frequencies approximately equal to $\omega_{10}-|x|, \omega_{10}-2|x|$, etc., where $x$ is the anharmonicity constant.

From the equations derived in the foregoing section it is easy to obtain, neglecting small anharmonic corrections for the dipole moments of transitions, the following expressions for multiphoton Rabi frequencies in the case of a Morse oscillator:

$$
\gamma^{(N)}=\frac{N^{1 / 2} \gamma_{01}^{N}}{[(N-1) !]^{3 / 2}|x|^{N-1}}
$$

The typical dependencies of multiphoton Rabi frequencies $(N=2-5)$ on the intensity of monochromatic radiation are shown in Figure 3.6. The oscillator parameters are given in the caption to this figure. It follows from the results of the foregoing section that from the values of the multiphoton Rabi frequencies one can estimate the half-widths of multiphoton resonances using Eq. (3.55). If for example, the anharmonicity constant $|x|=$ $2 \mathrm{~cm}^{-1}$, the radiation intensity is $10^{7} \mathrm{~W} / \mathrm{cm}^{2}$, and the dipole moment of the transition $v=0 \rightarrow v=1$ is 0.3 Debye, the half-width of the threephoton resonance is just $10^{-3} \mathrm{~cm}^{-1}$. Thus, it may be seen that the note made at the end of the foregoing section on the role of the nonmonochroma- 


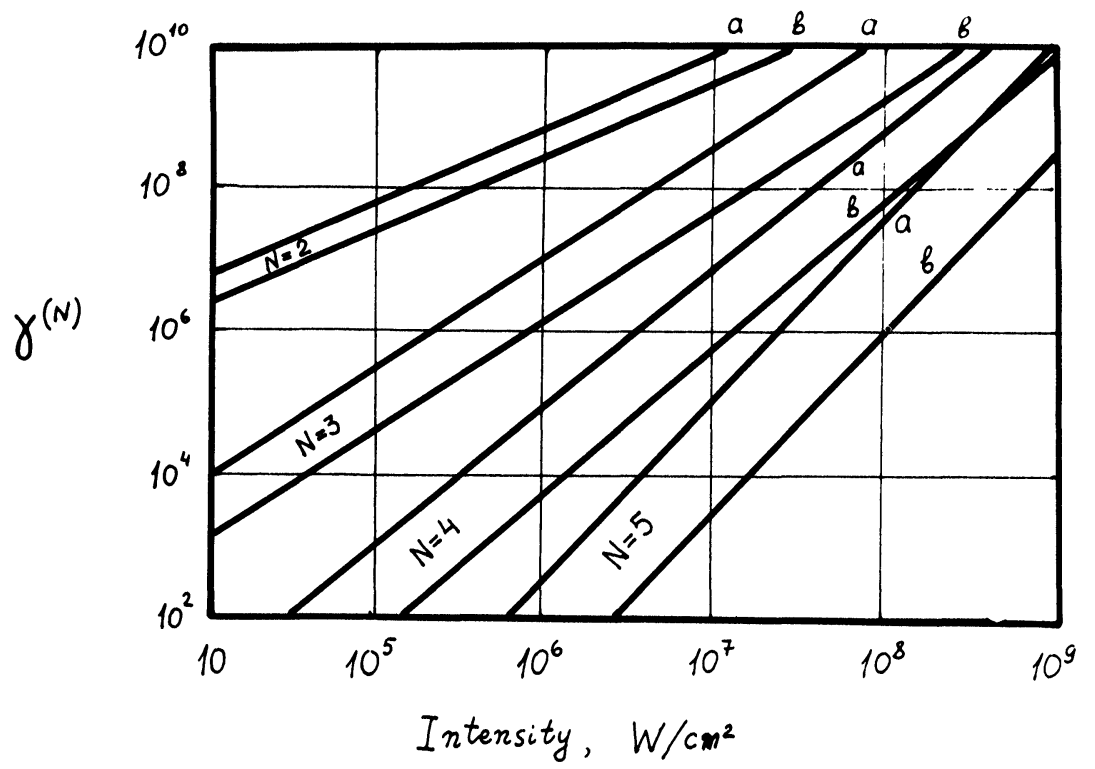

FIGURE 3.6 The dependencies of multiphoton Rabi frequencies on the radiation intensity at the following parameters of anharmonic oscillator: $\mu_{01}=0.3$ Debye; (a) $|x|=2$ $\mathrm{cm}^{-1} ; \quad$ (b) $|x|=5 \mathrm{~cm}^{-1}$.

ticity of laser radiation is really very essential for estimating the times required for these multiphoton transitions.

Now let us consider the dependence of the population $\rho_{v v}$ of the $v$-th oscillator level on the field frequency, particularly averaged over a long period of time. At the frequency of the $v$-photon resonance the value of $\overline{\rho_{v v}(t)}$ will be maximum and approximately equal to $\frac{1}{2}$, but peaks in $\overline{\rho_{v v}(t)}$ will be observed at the frequencies of other resonances too. An example illustrating this is given in Figure 3.7. This figure also shows the effect of the broadening of resonances as the field intensity increases.

When the field is so strong that the resonance half-width exceeds the anharmonicity constant, i.e.,

$$
(\Delta \Omega)^{(N)} \geq|x|
$$

the adjacent resonances become overlapped, and the frequency of multiphoton resonance is not yet distinguished. The condition (3.57), as the 


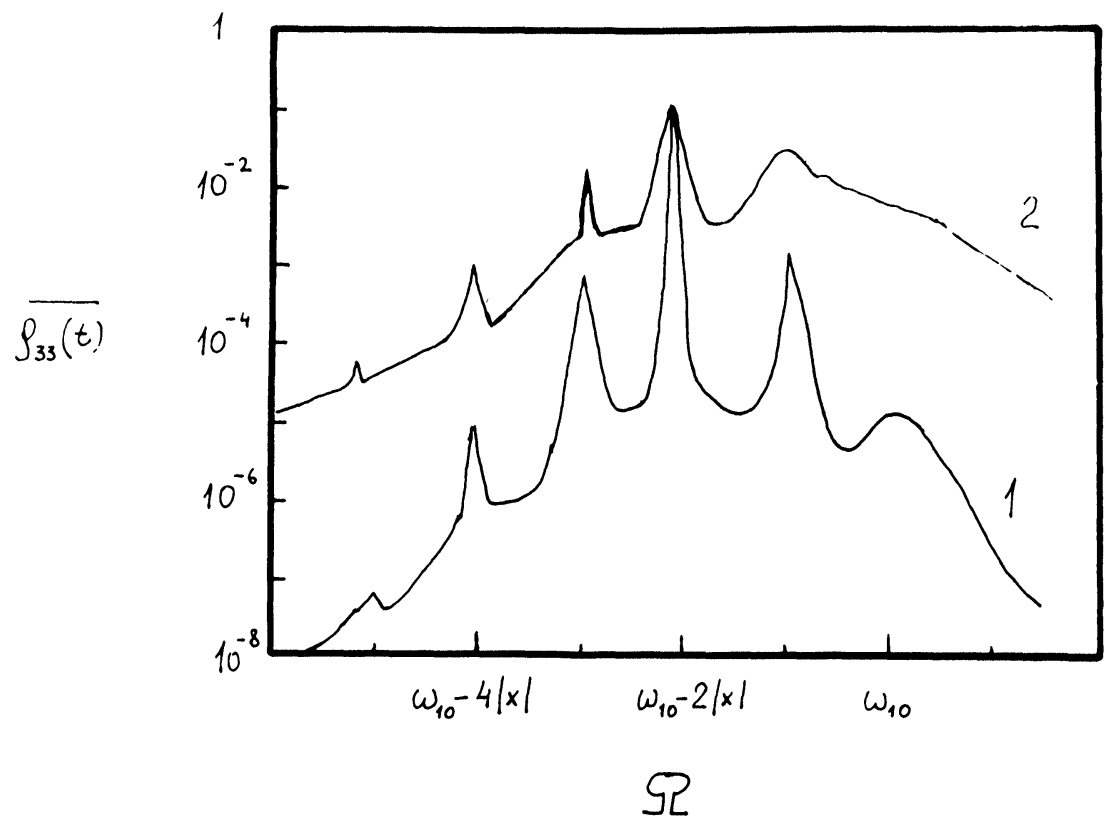

FIGURE 3.7 The dependence of the time-averaged population of third anharmonic-oscillator level on the exciting frequency. ${ }^{187}$ The parameter values are: curve $1: \gamma_{01} / 2|x|=0.1 ;$ curve 2: $\gamma_{01} / 2|x|=0.4$.

field amplitude grows, is not reached for all resonances simultaneously. Designating as $N_{\max }$ that maximum value of $N$, for which the condition (3.57) is just met at a given intensity, one often refers to the range of radiation frequencies $\Omega>\omega_{10}-N_{\max }|x|$ as the classical region. This means that in this frequency range the quantum features of the oscillator are only slightly important, and its behavior is close to the classical case. The promotion of a classical nonlinear oscillator by an external monochromatic field is a problem treated in many textbooks (see, for example, Ref. 188). The classical limit, however, is not of particular interest to us since, in the following experiments, the laser radiation intensity is not usually higher than $10^{8}-10^{9} \mathrm{~W} / \mathrm{cm}^{2}$. At the same time, for the oscillator parameters used for Figure 3.6, the equality, for example, $(\Delta \Omega)^{(3)}=2$ $\mathrm{cm}^{-1}$ is reached at the intensity of radiation $1.6 \times 10^{9} \mathrm{~W} / \mathrm{cm}^{2}$.

For the vibration-rotation molecular spectra, the sequence of transitions corresponding to the Morse oscillator may be approximately realized if 
one, for example, limits himself to choosing the transitions without a change in rotational quantum numbers. But it is easy to show that the frequencies make up an arithmetic progression for successive transitions within any given rotational branch, too. Let us take, for example, the transitions within the $P$ - or $R$-branches of spherical tops [see Eqs. (2.49) from Ref. 175], or within those of parallel bands of symmetrical tops [see Eqs. (2.51) from Ref. 175]. It may be seen that, neglecting the small change in the rotational constant, one can formally introduce the effective anharmonicity constant $|x|-B$. Thus, if one considers the $P$ - or $R$-branch separately, the anharmonicity constant apparently decreases. ${ }^{189,190}$ However, usually $B \ll|x|$ for molecules and the decrease has no particular effect on estimates. Otherwise, if one considers sequences of transitions in different bands, a wide variety of multiphoton resonances become possible. We shall turn to the discussion of this point in Section 5 of our next paper, where the role of some other effects will be discussed which arise from splittings of vibration-rotation states [see Ref. 175 (Sections 2.1.5 and 2.2.4)]. These splittings complicate the spectroscopic picture and, hence, make it necessary to consider more complex multilevel systems than those concerned in this Section.

\subsection{Incoherent excitation of quantum systems with relaxation}

In an analysis of the processes of absorption and stimulated emission, one may often hear the term transition rate. This value is expressed in $\mathrm{s}^{-1}$ and, in the case of a two-level system, is logically used to describe the dynamics of populations of the lower and upper levels in accordance with the following equations:

$$
\begin{aligned}
& \frac{d \rho_{00}}{d t}=-W\left(\rho_{00}-\rho_{11}\right) \\
& \frac{d \rho_{11}}{d t}=W\left(\rho_{00}-\rho_{11}\right)
\end{aligned}
$$

usually referred to as kinetic equations. It is trivial to solve Eqs. (3.58). If, for example, initially at $t=0$ the system is in the lower state, the solution has the form

$$
\rho_{00}=\frac{1}{2}[1+\exp (-2 W t)], \quad \rho_{11}=\frac{1}{2}[1-\exp (-2 W t)],
$$


i.e., the transition is saturated for the characteristic time $\tau_{\text {sat }}=\frac{1}{2} W$. However, the analysis carried out in Section 3.1 of the behavior of the twolevel system in a monochromatic field shows that it is hardly possible to describe the dynamics of coherent excitation in terms of transition rates. In particular, at short times, when the population of the upper level is negligible, it grows [see Eq. (3.19)] proportionally to $t^{2}$, whereas the second of Eqs. (3.59) gives $\rho_{11} \sim t$. Also, according to (3.59), the populations do not exhibit any oscillations but exponentially reach their mean values $\frac{1}{2}$.

\subsubsection{Conditions of incoherent excitation for two-level system}

The Eqs. (3.58) which are also referred to as the incoherent approximation are often a good approximation in the presence of some factors which lead to the broadening of spectra. In Ref. 175 (Section 2.6) we have already treated the effects responsible for spectral line broadening. Getting ahead, we may present a criterion when Eqs. (3.58) work well. This criterion requires that the line-width be much larger than the value of power broadening. In this section we shall treat the effect of both inhomogeneous (Doppler) and homogeneous (collisional) broadening. One more important case when kinetic equations may be used should also be pointed out. This is the situation when transitions into many close states are possible from the initial level, and it will be discussed in Section 3.7.

Let us begin with the excitation of a gas of two-level non-interacting molecules, with statistically distributed velocities, i.e., having different transition frequencies due to the Doppler effect. We shall be interested in the dependence on time of the excitation probability of the upper level averaged over all molecules. With the use of Eqs. (3.19) and (2.119) (from Ref. 175) this dependence can be easily written explicitly:

$$
\begin{gathered}
\left\langle\rho_{11}(t)\right\rangle=\frac{c}{\Omega}\left(\frac{M}{2 \pi k T}\right)^{1 / 2} 4 \gamma_{01}^{2} \int \frac{1}{(\Omega-\omega)^{2}+4 \gamma_{01}^{2}} \\
\sin ^{2}\left\{\frac{1}{2}\left[(\Omega-\omega)^{2}+4 \gamma_{01}^{2}\right]^{1 / 2} t\right\} \exp \left[-\frac{M(\Omega-\omega)^{2} c^{2}}{2 k T \Omega^{2}}\right] d \omega,
\end{gathered}
$$

where, for simplicity, the field frequency is taken to coincide with the center of the Doppler contour. The integral entering Eq. (3.60) contains two functions which depend on frequency and have their maximum at that of the field. In the cases when the width of these functions differ greatly, 
the variation of the broader function of frequency may be neglected, and we may pull it outside the integral.

If the Doppler half-width is much smaller than the value $2\left|\gamma_{01}\right|$ of power broadening the average population of the upper level behaves, of course, almost according to Eq. (3.19). But now we are only interested in the opposite case when

$$
(\Delta \omega)_{\text {Doppl }}=\frac{\Omega}{c}\left(\frac{2 k T}{M} \ln 2\right)^{1 / 2} \gg 2\left|\gamma_{01}\right|
$$

In this case, the integral in Eq. (3.60) can be expressed through an integral of the zero-order Bessel function which, in its turn, can be written conveniently as the series:

$$
\begin{gathered}
\left\langle\rho_{11}(t)\right\rangle \simeq \frac{c}{\Omega}\left(\frac{M}{2 \pi k T}\right)^{1 / 2} 4 \gamma_{01}^{2} \int \frac{1}{(\Omega-\omega)^{2}+4 \gamma_{01}^{2}} \\
\quad=\frac{2 \pi c}{\Omega}\left(\frac{M}{2 \pi k T}\right)^{1 / 2} \gamma_{01}^{2} \int_{0}^{t} J_{0}\left(2 \gamma_{01} \tau\right) \mathrm{d} \tau \\
=\frac{c}{\Omega}\left(\frac{2 \pi M}{k T}\right)^{1 / 2} \gamma_{01} \sum_{k=0}^{\infty}(-1)^{k} \frac{\left(\gamma_{01} \tau\right)^{2 k+1}}{(2 k+1)(k !)^{2}}
\end{gathered}
$$

The derived equation shows that, at small times, the average population of the upper levels now grows proportionally to time as given by Eqs. (3.58) with

$$
W=\frac{c}{\Omega}\left(\frac{2 \pi M}{k T}\right)^{1 / 2} \gamma_{01}^{2},
$$

though the population dynamics is not described by Eqs. (3.58) within the whole time interval.

The case of inhomogeneous Doppler broadening considered is of little interest for the goals of this series of papers but is illustrative. Indeed, with the radiation frequency $1000 \mathrm{~cm}^{-1}$ and the transition dipole moment 0.3 Debye, the condition (3.61) is realized only at very low radiation intensities $\leqslant 10 \mathrm{~W} / \mathrm{cm}^{2}$. For our purposes, it is more important to examine the role of collisional broadening, since in our discussions we shall come 
across some experiments in which the collisions between molecules in a gas have time to occur during the laser pulse. Besides, in the case of collisional broadening and at sufficiently frequent collisions, Eqs. (3.58) prove to be valid now within the whole time interval.

It is not, however, quite apparent how the collisional relaxation processes can be included, since so far we dealt with the Schrödinger equation or with the equations of motion for probability amplitudes only in view of isolated quantum systems. We have noted in Section 2.5.7 from Ref. 175 that the description using the density matrix is the most general statistical one. The formalism of a density matrix is well adapted to the description of relaxation processes. On the other hand, the equations for probability amplitudes may always be transformed into those for elements of the density matrix. In the case of a two-level system in particular, one can obtain the equations for the diagonal elements $\rho_{00}=a_{0}^{*} a_{0}$ and $\rho_{11}=a_{1}^{*} a_{1}$ of the density matrix as well as for nondiagonal ones $\rho_{01}=a_{0}^{*} a_{1}$ and $\rho_{10}$ $=a_{1}^{*} a_{0}$ starting from Eqs. (3.11). These equations of motion are

$$
\begin{aligned}
\frac{d \rho_{00}}{d t} & =i \gamma_{01}\left\{\rho_{01} \exp [i(\Omega-\omega) t]-\rho_{10} \exp [-i(\Omega-\omega) t]\right\} \\
\frac{d \rho_{11}}{d t} & =-i \gamma_{01}\left\{\rho_{01} \exp [i(\Omega-\omega) t]-\rho_{10} \exp [-i(\Omega-\omega) t]\right\} \\
\frac{d \rho_{01}}{d t} & =i \gamma_{01}\left(\rho_{00}-\rho_{11}\right) \exp [-i(\Omega-\omega) t] \\
\rho_{10} & =\rho_{01}^{*}
\end{aligned}
$$

The solutions of Eqs. (3.64) are, of course, absolutely identical to those of Eqs. (3.11). But Eqs. (3.64) are more convenient in view of the natural inclusion of terms which describe entering and leaving the lower and upper levels of the working transition due to the relaxation processes. Such terms should be introduced into equations for the diagonal as well as nondiagonal elements of the density matrix. In addition, a term of type (2.117) (see Ref. 175) enters the equations for nondiagonal elements which describes their relaxation to the zero equilibrium value (see the discussion in Section 2.5.7 from Ref. 175). We do not write out here the general equations which are usually referred to as the Bloch equations. ${ }^{191}$ In the general case, the solutions of these equations are rather cumbersome, and they are studied in some manuals (see, for example, Refs. 176 and 180). However, here is another point of interest for us. Let us look at the kinetic equations (3.58) for the populations of levels. If one assumes that these equations 
are a good approximation, then it is not difficult, in principle, to include the relaxation terms into them. The only process which cannot be included directly into Eqs. (3.58) is the phase or transversal relaxation of the densitymatrix nondiagonal elements. Therefore, it is appropriate to ask in which cases the solutions of the equations for density-matrix elements, in the presence of phase relaxation only, are close to the solution (3.59) of kinetic equations.

Thus, let us introduce into Eqs. (3.64) the terms which describe relaxation of nondiagonal elements to zero and suggest for simplicity that the field frequency is equal to the transition one. We come to

$$
\begin{aligned}
& \frac{d \rho_{00}}{d t}=i \gamma_{01}\left(\rho_{01}-\rho_{10}\right) \\
& \frac{d \rho_{11}}{d t}=-i \gamma_{01}\left(\rho_{01}-\rho_{10}\right) \\
& \frac{d \rho_{01}}{d t}=i \gamma_{01}\left(\rho_{00}-\rho_{11}\right)-\frac{1}{T_{2}} \rho_{01} \\
& \frac{d \rho_{10}}{d t}=-i \gamma_{01}\left(\rho_{00}-\rho_{11}\right)-\frac{1}{T_{2}} \rho_{10}
\end{aligned}
$$

We are interested in the solution of these equations when the system is initially at $t=0$ in the lower state, i.e., $\rho_{00}(t=0)=1, \rho_{11}(t=0)=$ $\rho_{01}(t=0)=\rho_{10}(t=0)=0$. The solution can be found easily and for the diagonal elements of the density matrix, in particular, it has the following form:

$$
\begin{aligned}
\rho_{00}= & \frac{1}{2}+\frac{1}{2} \exp \left(-\frac{t}{2 T_{2}}\right)\left\{\operatorname{ch}\left[\left(1-16 \gamma_{01}^{2} T_{2}^{2}\right)^{1 / 2} \frac{t}{2 T_{2}}\right]\right. \\
& \left.+\frac{1}{\left(1-16 \gamma_{01}^{2} T_{2}^{2}\right)^{1 / 2}} \operatorname{sh}\left[\left(1-16 \gamma_{01}^{2} T_{2}^{2}\right)^{1 / 2} \frac{t}{2 T_{2}}\right]\right\} \\
\rho_{11}= & \frac{1}{2}-\frac{1}{2} \exp \left(-\frac{t}{2 T_{2}}\right)\left\{\operatorname{ch}\left[\left(1-16 \gamma_{01}^{2} T_{2}^{2}\right)^{1 / 2} \frac{t}{2 T_{2}}\right]\right. \\
& \left.+\frac{1}{\left(1-16 \gamma_{01}^{2} T_{2}^{2}\right)^{1 / 2}} \operatorname{sh}\left[\left(1-16 \gamma_{01}^{2} T_{2}^{2}\right)^{1 / 2} \frac{t}{2 T_{2}}\right]\right\}
\end{aligned}
$$


The behavior of this obtained solution is different depending on the relation between the values $4 \gamma_{01}$ and $T_{2}^{-1}$. With the condition $4\left|\gamma_{01}\right|>$ $T_{2}^{-1}$, the population oscillations are observed which decay with the characteristic time $2 T_{2}$, the populations tending to their average values equal to $\frac{1}{2}$. If the opposite condition $4\left|\gamma_{01}\right|<T_{2}^{-1}$ is fulfilled, the equilibration of populations occurs without oscillations, and this process is described by two exponents. Finally, if $4\left|\gamma_{01}\right| \ll T_{2}^{-1}$, then only one exponent plays a significant role:

$$
\rho_{00} \simeq \frac{1}{2}+\frac{1}{2} \exp \left(-4 \gamma_{01}^{2} T_{2} t\right), \quad \rho_{11} \simeq \frac{1}{2}-\frac{1}{2} \exp \left(-4 \gamma_{01}^{2} T_{2} t\right)
$$

Comparing Eqs. (3.67) with Eqs. (3.59) one may conclude that the solution of density-matrix equations in case $4\left|\gamma_{01}\right| \ll T_{2}^{-1}$ coincides with that of kinetic equations, if

$$
W=2 \gamma_{01}^{2} T_{2}
$$

is taken. Thus, sufficiently frequent phase-interrupting collisions destroy the coherence of interaction of the two-level system with the field.

In Section 2.6 from our previous paper (Ref. 175), when discussing spectral-line broadenings, we introduced the value of an absorption crosssection [see Eq. (2.122) from Ref. 175]. Now one may correlate the transition rate $W$ with the absorption cross-section. The cross-section includes the factor $G_{\Omega}$ which gives the line shape. In the considered specific cases when the field frequency lies in the absorption contour center, the factor $G_{\Omega}$ can be found from formulas from Ref. 175, Eq. (2.119) for an inhomogeneously broadened line and Eq. (2.121) with $(\Delta \omega)_{\text {hom }}=T_{2}^{-1}$ for a homogeneously broadened line, if, in these equations, $\omega=\omega_{0}$ is taken. It is easy to obtain from Eqs. (3.63) and (3.68) that, for both the cases considered,

$$
W=\frac{\sigma I}{\hbar \Omega}=\sigma P
$$

where $P$ denotes the radiation intensity expressed in terms of photon $\times$ $\mathrm{cm}^{-2} \times \mathrm{s}^{-1}$. This formula for the transition rate remains valid for the more general case, too, when the field frequency is shifted from the center of the absorption contour, but one should not forget that the concept of a 
transition rate itself makes sense provided that rather moderate fields are used for excitation.

From the analysis carried out, one important conclusion can be drawn. Whereas, under coherent excitation, the characteristic transition time is inversely proportional to the electric component of field, this time is inversely proportional to the intensity of radiation under the incoherent excitation. Accordingly, for the saturation of a transition under incoherent excitation, the fluence of the excitation wave is of importance, and the characteristic required value $\Phi_{\text {sat }}=\hbar \Omega / 2 \sigma$ is called the saturation energy.

\subsubsection{Effect of rotational bottleneck}

In Section 2.5 from Ref. 175, when discussing collisional relaxation processes in gases, we distinguished the rotational relaxation as the fastest of the processes which change populations of vibration-rotation molecular levels. In this section we want to treat the kinetics of excitation of a quasitwo-level vibrational transition in the presence of rotational relaxation, taking into consideration the real distribution of molecules over the rotational sublevels of the ground vibrational state. For simplicity, we shall neglect the initial population of the upper vibratational state and assume that the radiation field effectively interacts with only one vibration-rotation transition, i.e., with a really small fraction of all molecules in the gas. The last assumption actually means that the value of power broadening is much less than the characteristic difference of frequencies of two adjacent transitions in the vibration-rotation spectrum. But, as will be seen below, this assumption is not absolutely necessary.

Thus, we consider (see Figure 3.8) the field frequency to be resonant to one of the transitions in the vibration-rotation spectrum and assume that the direct excitation from the rest of rotational sublevels may be neglected. The molecules originally in non-resonant levels are allowed to be excited effectively only if they enter the resonant level by the process of rotational relaxation. Now let us treat what the equations are for the adequate description of the kinetics of the process. Since the very sense of the problem makes for insight into the behavior of a system during time intervals longer than the rotational-relaxation time, it is natural to use the kinetic equations for the populations of levels. Indeed, the rotational-relaxation time is at least no less than the time $T_{2}$ of the phase relaxation. If the value of power broadening is much smaller than $T_{2}^{-1}$, the kinetic equations, as has been shown in the foregoing section, give a good approximation for describing the excitation of the working vibration-rotation transition. On the other 


\section{Rotational \\ leviels}

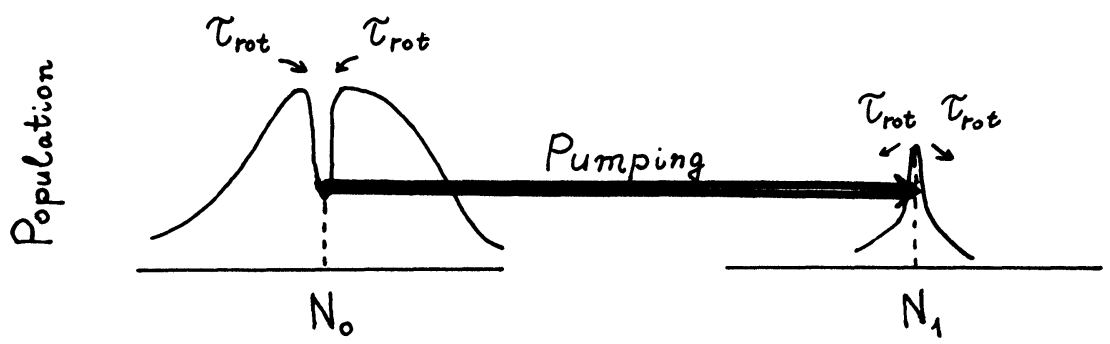

FIGURE 3.8 The role of rotational relaxation in the process of excitation of molecular gas when the field interacts with one vibration-rotation transition.

hand, any other ratio between the value of power broadening and the phase relaxation time forces the populations of working levels, as follows from Eqs. (3.66), to tend toward their equilibrium values $\frac{1}{2}$ during a time about $T_{2}$, i.e., the use of kinetic equations which, for this case, is not quite correct physically should not, nevertheless, lead to any essential error.

Now let us discuss a possible way to describe the rotational relaxation in our specific situation. In Section 2.5.3 of Ref. 175 we discussed various approximations to describe relaxation processes phenomenologically. For our case, the simplest linear approximation is quite suitable. The arguments for using the linear approximation are rather simple. The radiation, as it acts on one vibration-rotation transition, is able to create strong nonequilibria only in active vibration-rotation levels. (According to Figure 3.8, we shall designate their populations as $N_{0}$ and $N_{1}$.) Since the fraction of molecules interacting with the radiation is small, the populations of the rest of the levels differ just slightly from their equilibrium values, and this fact justifies the description of their populations within Eqs. (2.109) from Ref. 175.

Further, let us consider one of the vibrational states, say the lower one, and designate the populations of sublevels which do not interact with radiation as $N_{j}$ and the total population of the lower vibrational state as $\tilde{N}_{0}$. The equilibrium populations of different rotational sublevels can be represented as $F_{j} \tilde{N}_{0}$, where $F_{j}$ are the Boltzmann factors (see Section 2.5.1 from Ref. 175). Within the linear approximation, the relaxation term for each rotational sublevel may be written out in the following form: 


$$
\frac{d N_{j}}{d t}=\frac{F_{j} \tilde{N}_{0}-N_{j}}{\tau_{\text {rot }}^{(j)}}
$$

On the other hand, the process of rotational relaxation cannot change the total population of the vibrational state, i.e.,

$$
N_{0}+\sum_{j} N_{j}=\tilde{N}_{0}, \quad \frac{d \tilde{N}_{0}}{d t}=0,
$$

and, if one assumes that the rotational-relaxation rates are approximately equal for all the really populated rotational sublevels, then the following sequence of equations will occur:

$$
\begin{aligned}
\frac{d N_{0}}{d t} & =-\frac{d}{d t} \sum_{j} N_{j} \simeq-\frac{1}{\tau_{\text {rot }}} \sum_{j}\left(F_{j} \tilde{N}_{0}-N_{j}\right) \\
& =-\frac{1}{\tau_{\text {rot }}}\left[\left(1-F_{0}\right) \tilde{N}_{0}-\left(\tilde{N}_{0}-N_{0}\right)\right]=\frac{F_{0} \tilde{N}_{0}-N_{0}}{\tau_{\text {rot }}},
\end{aligned}
$$

i.e., the same relaxation equation as (3.70) must be valid for a distinguished sublevel which is perhaps strongly perturbed.

Now it is not difficult to write out the complete set of equations which includes the interaction of radiation with the active transition as well as the process of rotational relaxation. This is the set of four equations for two populations $N_{0}$ and $N_{1}$ of the active vibration-rotation sublevels and two total populations $N_{0}$ and $\tilde{N}_{1}$ of vibrational states. Of course, the equations for total populations do not include relaxation terms, and the full set of equations has the form

$$
\begin{aligned}
& \frac{d N_{0}}{d t}=-\sigma P\left(N_{0}-N_{1}\right)+\frac{F \tilde{N}_{0}-N_{0}}{\tau_{\text {rot }}} \\
& \frac{d N_{1}}{d t}=\sigma P\left(N_{0}-N_{1}\right)+\frac{F \tilde{N}_{1}-N_{1}}{\tau_{\text {rot }}} \\
& \frac{d \tilde{N}_{0}}{d t}=-\sigma P\left(N_{0}-N_{1}\right) \\
& \frac{d \tilde{N}_{1}}{d t}=\sigma P\left(N_{0}-N_{1}\right)
\end{aligned}
$$


where, for simplicity, the rotational relaxation times as well as the population factors are taken to be equal for the lower and upper states, and $\sigma$ denotes the absorption cross section of the active vibration-rotation transition. We are not writing out here the general solution of Eqs. (3.73) obtained in Ref. 192 assuming sequential onset of the radiation wave. This solution, which can be expressed in terms of two exponents, is rather cumbersome, but with $F \ll 1$ it may be essentially simplified and, for total populations $\tilde{N}_{0}$ and $\tilde{N}_{1}$ of vibrational states, leads to the following approximate expressions which resemble solution (3.59) of the simplest kinetic equations:

$$
\tilde{N}_{0} \simeq \frac{1}{2}[1+\exp (-2 w t)], \quad \tilde{N}_{1} \simeq \frac{1}{2}[1-\exp (-2 w t)],
$$

where the effective vibrational excitation rate is

$$
w=\frac{\sigma P F}{1+2 \sigma P \tau_{\text {rot }}}
$$

The value $(2 w)^{-1}$ gives the characteristic time $\tau_{\text {sat }}$ of saturation of the whole vibrational transition. At low radiation intensities when the condition $\sigma P \tau_{\text {rot }} \ll 1$ is fulfilled, we have

$$
\tau_{\text {sat }} \simeq(2 \sigma P F)^{-1},
$$

i.e., the value $\tau_{\text {sat }}$ is $1 / F$ times larger than the time of saturation of an active vibration-rotation transition alone. As the intensity increases, the time of saturation decreases and, with the condition $\sigma P \tau_{\text {rot }} \gg 1$ fulfilled, reaches its minimum value

$$
\tau_{\text {sat }}^{\min } \simeq \tau_{\text {rot }} / F
$$

It is this last property putting the limit on the excitation rate that is usually referred to as the effect of rotational bottleneck. Physically this effect is rather obvious. The active transition is quickly saturated by high-intensity radiation. During an interval of time about $\tau_{\text {rot }}$ the molecules having been excited into the upper active vibration-rotation level relax to other rotational sublevels, and a small new portion of molecules about $F$ enters the lower active one, from where it is quickly excited upwards, etc. 
We assumed above that the radiation effectively interacts only with one vibration-rotation transition. Actually, for complex vibration-rotation molecular spectra, the field with a finite spectral width can resonantly interact with a group of transitions. For this case, the obtained equations remain valid although the population factor should be treated in some different way (to avoid confusion we shall use the notation $f$ for it). Now, the value $f$ will denote the relative resulting population of all rotational sublevels interacting with the radiation. The $f$ value understood in this way cannot be always easily estimated theoretically. From the analysis performed, however, a rather simple method follows which enables us to determine it experimentally. One should measure the saturation energy with a short radiation pulse, the duration of which is much shorter than the rotationalrelaxation time, and also with a long radiation pulse, the duration of which is much longer that the rotational-relaxation time. Then, it follows from the discussion on Eq. (3.76), the $f$ value can be obtained from the ratio of the saturation energies in the two experiments. This method had been realized in Ref. 193 for the molecule $\mathrm{C}_{2} \mathrm{~F}_{3} \mathrm{Cl}$.

There is one more important point to be mentioned. For molecules with closely spaced vibrational-rotational lines, even within our simplified quasitwo-level model, the factor $f$ must grow with radiation intensity, since the value of power broadening increases. This effect, no doubt, becomes important when the value of the power broadening is greater than the width of the radiation spectrum (see the estimates at the end of Section 3.2.1). In this case, one can roughly estimate the factor $f$ as the ratio of the value of power broadening to the half-width $(\Delta v) / 2$ of that rotational branch where the radiation frequency lies, i.e.,

$$
f \sim 4\left|\gamma_{01}\right| /(\Delta v)
$$

The obtained expressions can be modified, of course, with this effect being taken into account. But, this is hardly worthwhile, since the experiments demonstrate convincingly that, as the molecules are acted upon by rather strong laser fields even under collisionless conditions, an essentially larger fraction of molecules is excited than that allowed by Eq. (3.78). We shall return to discussing this problem more than once. One can probably gain some insight from the parallel observation that the higher vibrational states than the fundamental one are excited, too, i.e., under sufficiently strong fields, the molecule no longer behaves as the quasi-two-level system examined in this section. 


\subsection{Excitation from a discrete level into a quasicontinuous band}

In Section 2.3.4 of our paper (Ref. 175), we discussed the features of the spectrum of vibrational transitions in the region of stochasticity of vibrational motion. In this region, the spectrum becomes, in fact, quasicontinuous because of the enormous density of states and strong mixing of the harmonic wavefunctions due to anharmonicity. In other words, many transitions become possible from a vibration-rotation state, their frequencies being very close and covering a rather wide spectral range. It is evident that, in the presence of a large number of transitions, the two-level approximation is invalid if the value of power broadening becomes comparable to or exceeds the typical differences between frequencies of adjacent transitions. And what is more, the description with the use of kinetic equations proves to be a good approximation for this case. To demonstrate this we shall treat in this section the dynamics of excitation from a discrete state into a band of closely spaced levels (see Figure 3.9). Such a process is an elementary act of excitation into the vibrational quasicontinuum by radiation.

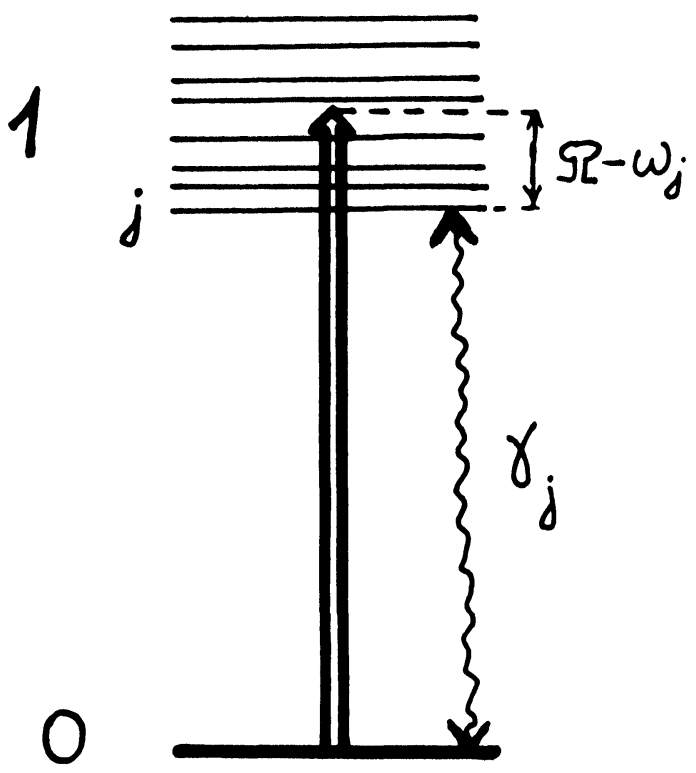

FIGURE 3.9 Transitions from a discrete level to a band of closely spaced states. 


\subsubsection{Derivation of kinetic equations}

The equations for the probability amplitudes of the lower level $a_{0}$ and the levels in the band $a_{1 j}$ have, within the rotating-wave approximation, the following form:

$$
\begin{aligned}
& \frac{d a_{0}}{d t}=i \sum_{j} \gamma_{j} a_{1 j} \exp \left[i\left(\Omega-\omega_{j}\right) t\right] \\
& \frac{d a_{1 j}}{d t}=i \gamma_{j} a_{0} \exp \left[-i\left(\Omega-\omega_{j}\right) t\right]
\end{aligned}
$$

Here $\omega_{j}$ is the frequency of transition from the $j$-th level in the band to the lower state, $\gamma_{j}=\langle 0|\hat{\mu}| 1, j\rangle \mathscr{E} / 2 \hbar$ is the corresponding Rabi frequency, and the indexes 0 and 1 relating to the lower level and the whole manifold of upper levels are omitted for brevity.

Eqs. (3.79) cannot, of course, be solved explicitly for arbitrary distributions of dipole-moment matrix elements and frequencies over various transitions. We shall consider the only known example for which it is possible to get an analytical solution. ${ }^{194}$ This is the case when the levels in the band are equidistant, i.e.,

$$
\omega_{j}=\omega_{0}+j / \hbar \rho \quad(-\infty<j<\infty),
$$

where $\rho^{-1}$ is the distance between adjacent levels, and when the dependence of $\gamma_{j}^{2}$ on $j$ is Lorentzian, i.e.,

$$
\gamma_{j}^{2}=\frac{\delta}{\pi \hbar \rho} \frac{\gamma^{2}}{\delta^{2}+j^{2} / \hbar^{2} \rho^{2}} \tanh (\pi \delta \hbar \rho)
$$

Here $\delta$ is the half-width of the $\gamma_{j}^{2}$ curve, and the normalization is chosen so that

$$
\sum_{j=-\infty}^{\infty} \gamma_{j}^{2}=\gamma^{2}
$$

i.e., the value $\gamma$ may be called the integral Rabi frequency.

As usual, we shall be interested in the solution of Eqs. (3.79) for the 
probability amplitudes generated by stepwise switching on of the field, and with the following initial conditions:

$$
a_{0}(t=0)=1, \quad a_{1 j}(t=0)=0
$$

As the general theory dictates, the solution we are interested in can be obtained if one finds the dressed states and makes up their superposition complying with the initial conditions (3.83). However, in the particular case under consideration, it is simpler, first, to transform Eqs. (3.79) to one integro-differential equation which includes only the probability amplitude $a_{0}(t)$. This equation can easily be derived if one formally integrates Eqs. (3.79) for $a_{1 j}(t)$ and substitutes the result into the first equation. Doing this one comes to the following equation:

$$
\begin{aligned}
& \frac{d a_{0}}{d t}+\int_{0}^{t} a_{0}(\tau) K(t-\tau) d \tau=0, \\
& K(t-\tau)=\sum_{j} \gamma_{j}^{2} \exp \left[i\left(\Omega-\omega_{j}\right)(t-\tau)\right],
\end{aligned}
$$

the initial conditions are

$$
a_{0}(t=0)=1, \quad \frac{d a_{0}}{d t}(t=0)=0,
$$

using Eqs. (3.80) and (3.81) for $\omega_{j}$ and $\gamma_{j}^{2}$, the kernel comes to

$$
\begin{aligned}
& K(z)=\gamma^{2} \exp \left[i\left(\Omega-\omega_{0}\right) z\right][\operatorname{ch} \delta z-\tanh (\pi \delta \hbar \rho) \operatorname{sh} \delta z], \quad 0 \leqslant z \leqslant 2 \pi \hbar \rho \\
& K(z+2 \pi \hbar \rho)=K(z) \exp \left[i\left(\Omega-\omega_{0}\right) z\right]
\end{aligned}
$$

A standard technique for solving equations like (3.84) is to use the Laplace transformation. We will not trace here the intermediate calculations and shall write out the solution in its final form which may be checked directly through substituting. This solution has a rather complex structure, and all the necessary equations are given in Table 3.1. The solution, of course, depends on the detuning $\Delta=\Omega-\omega_{0}$ of the field frequency with respect to the center of the Lorentzian contour, and it includes the roots 


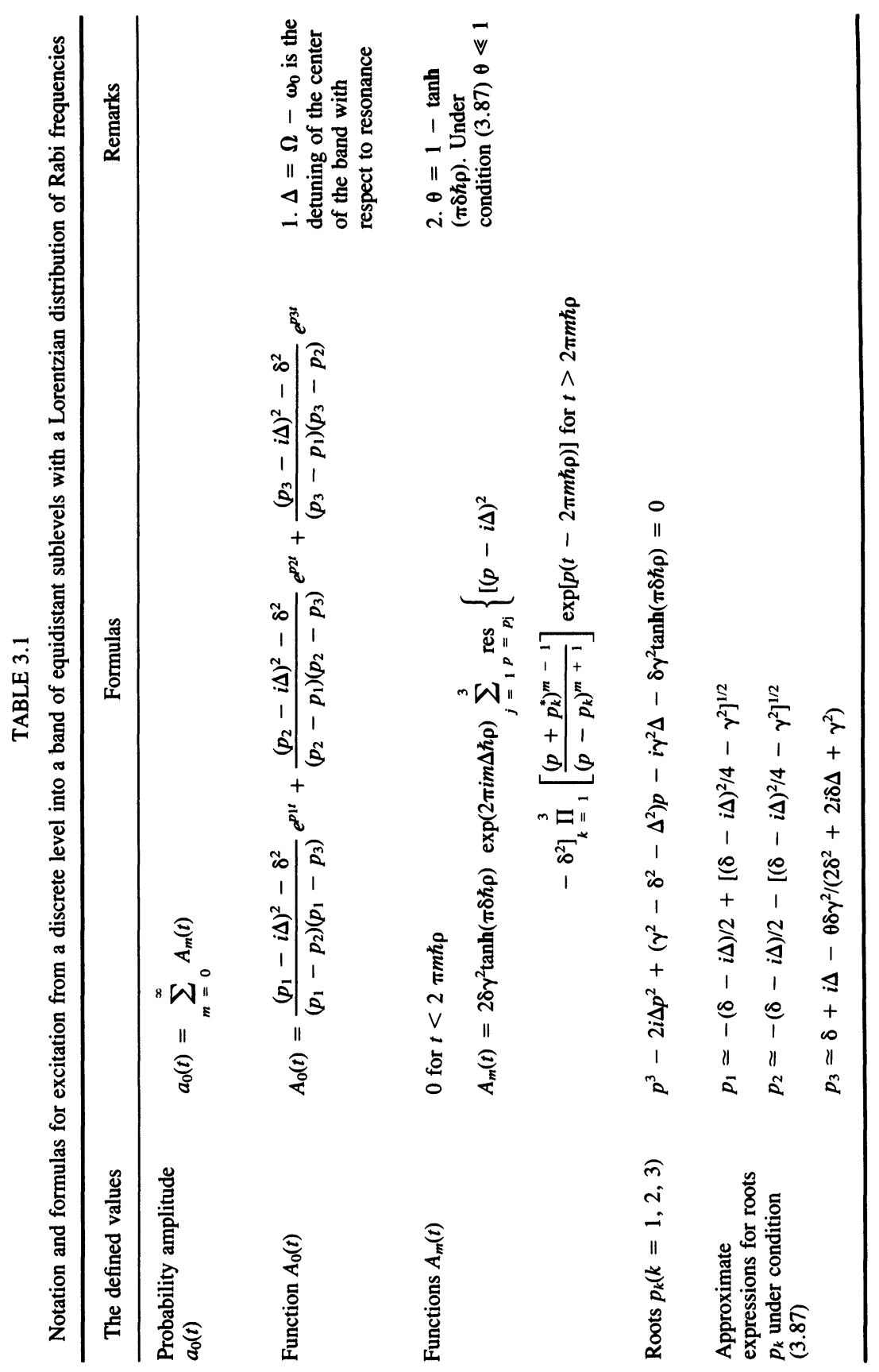


of the cubic equation written out in the fourth line of Table 3.1. Of physical interest for us is the situation when the half-width of the transition spectrum exceeds the distance between adjacent levels in the band, i.e., the condition

$$
\delta>1 / \hbar \rho
$$

is met. The solution comprises with this condition the small parameter $\theta$ $=1-\tanh (\pi \delta \hbar \rho) \ll 1$, and, for the roots of the cubic equations, there are valid and rather simple approximating formulas written out in the fifth line of Table 3.1.

Let us now examine the behavior of the probability amplitude within the time interval $0<t<2 \pi \hbar \rho$ when $a_{0}(t)=A_{0}(t)$ (see Table 3.1). Neglecting the terms which are small by the parameter $\theta$, one comes to the following equation:

$$
a_{0}(t) \simeq \frac{r_{1}+\delta-i \Delta}{r_{1}-r_{2}} e^{r_{1} t}+\frac{r_{2}+\delta-i \Delta}{r_{2}-r_{1}} e^{r_{2} t}
$$

where $r_{1,2}=-(\delta-i \Delta) / 2 \pm\left[(\delta-i \Delta)^{2} / 4-\gamma^{2}\right]^{1 / 2}$. If the field frequency coincides with that of the center of the transition contour, i.e., $\Delta=0$, then the obtained equation is similar in structure to Eqs. (3.66) which describe the dynamics of a two-level system in the presence of phase relaxation. As in that case, if the value $2 \gamma$ exceeds the half-width of the contour of transitions, then the damped oscillations will exhibit the dynamics of system and, in the opposite case when $2 \gamma \ll \delta$, the population of the lower level decays exponentially according to

$$
\rho_{00}=a_{0}^{*} a_{0} \simeq \exp \left(-\frac{2 \gamma^{2}}{\delta} t\right)
$$

With the detuning of the field frequency from the center of the contour of transitions, i.e., with $\Delta \neq 0$, two regimes are also possible, the damped oscillations and the purely exponential decay. As the criterion of the purely exponential decay, the condition

$$
\gamma \ll|\delta-i \Delta|
$$


serves, and, in this case,

$$
\rho_{00}(t) \simeq \exp \left(-\frac{2 \gamma^{2} \delta}{\delta^{2}+\Delta^{2}} t\right)
$$

With the purely exponential decay of the population of the lower level one probably wishes to present the transition rate

$$
W=\frac{2 \delta \gamma^{2}}{\delta^{2}+\Delta^{2}}
$$

which is proportional to $\gamma^{2}$ and, hence, the radiation intensity in terms of the absorption cross-section at the frequency of field. By introducing the integral square of the dipole moment $\mu^{2}$ we find that

$$
W=\frac{\mu^{2} \mathscr{E}^{2}}{2 \hbar^{2}} \frac{\delta}{\delta^{2}+\Delta^{2}}=\left\{\frac{4 \pi \mu^{2} \Omega}{\hbar c} \frac{\delta}{\pi\left[\delta^{2}+(\Omega-\omega)^{2}\right]}\right\} \frac{I}{\hbar \Omega}
$$

In Section 2.6 from Ref. 175 we introduced the absorption cross section (2.122) (from Ref. 175) for broadened lines. In that case the transition spectrum was continuous but it is discrete in the case under treatment. Nevertheless, it is easily seen that the value $\left(4 \pi \mu^{2} \Omega / \hbar c\right)\left\{\delta / \pi\left[\delta^{2}+(\Omega\right.\right.$ $\left.\left.-\omega)^{2}\right]\right\}$ would be just the natural generalization of the absorption crosssection for our quasicontinuous spectrum of transitions, if the substructure were ignored and the line-shape $G_{\Omega}=\delta / \pi\left[\delta^{2}+(\Omega-\omega)^{2}\right]$ introduced.

It should also be noted that the transitions from a discrete level occur into that range of the quasicontinuous spectrum which is resonant to the field frequency. This is mandated by the energy-conservation law and, from the uncertainty principle, the width of the excitation range within an order of magnitude is

$$
\Delta E \sim \hbar / W
$$

This conclusion follows from rather general quantum-mechanical principles, but it can be drawn directly from the solution of Eqs. (3.79) too. 


\subsubsection{Validity of the kinetic equations}

So far we have considered the population dynamics of the lower level only within the time interval $0<t<2 \pi \hbar \rho$ and concluded that, at comparatively weak fields, this dynamics is well described by a kinetic equation like

$$
\frac{d \rho_{00}}{d t}=-\sigma P \rho_{00}
$$

Now we want to clear up when it is sufficient to consider only this time interval. This obviously requires that the population of the lower level essentially decay during the time of order $2 \pi \hbar \rho$, i.e., the condition

$$
\left|a_{0}(2 \pi \hbar \rho)\right| \ll 1
$$

be valid. Rewriting the transition rate $W$ (3.92) with the use of Eq. (3.81) in terms of the Rabi frequency $\gamma_{\text {res }}$ near the resonance, one comes to the following natural requirement

$$
\gamma_{\text {res }}>1 / \hbar \rho,
$$

i.e., as discussed at the beginning of this Section, the value of power broadening evaluated at an individual near-resonant transition must exceed the distance between adjacent levels in the band. The condition (3.97), in addition to (3.90), serves as the second criterion for validity of the kinetic equation (3.95). This condition causes, in fact, the discrete structure of the transition spectrum to be unessential, and one actually may speak about a continuity of spectrum.

We now turn to particular estimates. Let the integral dipole moment of the level-band transitions

$$
\mu=\left(\sum_{j} \mu_{j}^{2}\right)^{1 / 2}
$$

be 0.3 Debye. From the mechanisms of stochastization of vibrational motion in molecules as discussed in Section 2.3 from Ref. 175 it is clear that the band widths in the vibrational quasicontinuum are typically $10-100$ 
$\mathrm{cm}^{-1}$. Therefore, the first condition (3.90) necesssary for the validity of kinetic equations requires that the radiation intensity be less than $10^{10} \mathrm{~W} /$ $\mathrm{cm}^{2}$. The typical intensities of laser radiation used in the experiments discussed in this series of papers are ordinarily much smaller than this value.

The second criterion for using the kinetic equations put requirements on both the lower limit of intensity and the density of states. One should keep in mind that, with the integral Rabi frequency fixed, the value $\gamma_{\text {res }}$ is inversely proportional to $\rho^{1 / 2}$. Let, for example, the field frequency lie near the center of the Lorentzian contour of transitions. Then one may find that the condition (3.97) transforms into

$$
\gamma^{2} \hbar \rho>\pi \delta
$$

Take for estimates $\delta=10 \mathrm{~cm}^{-1}, \mu=0.3$ Debye, and the radiation intensity $10^{7} \mathrm{~W} / \mathrm{cm}^{2}$. Then the condition (3.99) is fulfilled when the density of states exceeds $10^{3} \mathrm{~cm}$ corresponding, for example, to the density of vibrational states of the molecule $\mathrm{SF}_{6}$ at the energy $\sim 5000 \mathrm{~cm}^{-1}$. As will be seen below, when discussing experimental results, the value of radiation intensity taken is rather typical for observing the excitation of molecules into the vibrational quasicontinuum. On the other hand, the estimated value of the state denisty seem also probable for the lower energy limits of stochasticity regions in molecules. From this it follows that the description of the dynamics of excitation in the vibrational quasicontinuum within the kinetic equations is perhaps a rather good approximation. Furthermore, it should be added that the usual width of laser-radiation spectrum obviously reduces the condition on the density of states required for using the kinetic equations.

\subsection{Excitation of the vibrational quasicontinuum}

In the foregoing section we have established the conditions in which the radiation field produces the exponential decay of a discrete level into a quasicontinuous spectrum. The same conditions are required, in fact, for using the kinetic equations in the case of a more complex system when resonant transitions occur between two bands of the quasicontinuous spectrum (see Figure 3.10). 


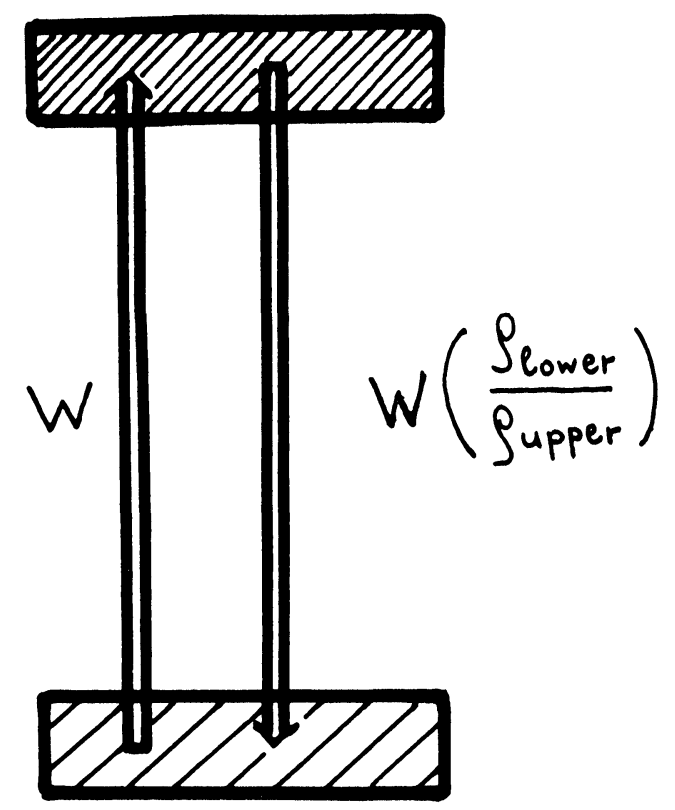

FIGURE 3.10 The relationship between the rates of upward and downward transitions between two quasicontinuous bands.

\subsubsection{Kinetic equations for stimulated transitions in vibrational quasicontinuum}

We shall not discuss here the substantiation of kinetic equations for this case. (The reader may turn to Refs. 195-197.) In this section we shall use the kinetic equations without any additional reservations, but first we want to focus our attention on some important physical points.

If at the initial instant of time the system is at some level of, say, the lower band, then the radiation produces transitions into a narrow part of the upper band. As has been mentioned in the foregoing Section, the width of this part is of the order of the inverse transition rate. Further, we should also take into account the downward transitions which led to populating the narrow part of the spectrum in the lower band. Therefore, thinking about the populations which enter the kinetic equations one should consider the total populations summed over the resonant parts of bands. (We shall designate them as $z_{\text {lower }}$ and $z_{\text {upper. }}$.) On the other hand, the description of 
the excitation dynamics in terms of total populations must presuppose that all the levels are rather equivalent, i.e., the transition cross-sections for them are approximately equal. In Section 2.3.4 from Ref. 175, when discussing the spectra in the vibrational quasicontinuum, we noted that the dipole moments of transitions, besides their regular average change with the energy, according to Eqs. (2.83) from Ref. 175, may also undergo irregular variations from transition to transition. It is easy to understand (see, for example, Ref. 198) a rough criterion for when it is possible to use the averaged dipole moments. It is necessary that the characteristic spectral scale of dipole-moment fluctuations be smaller than the inverse transition rate which fixes the width of the populated part of the spectrum. We shall assume that this criterion is fulfilled. Then it is physically justified to use the averaged value of $\mu^{2}$, through which one can naturally introduce the cross-section, as was done in Section 2.6 of Ref. 175 as well as in the foregoing one.

Another important point concerns the ratio between the rates of upward and downward transitions. In Section 3.5, discussing the dynamics of incoherent excitation of a two-level system, we came, in fact, to Eqs. (3.58) which exhibit equal rates of upward and downward transitions. In the foregoing Section while discussing the decay of a level into the quasicontinuous spectrum, we have shown that it is described by Eq. (3.95) which does not include downward transitions. It may easily be understood that these results reflect two specific ratios between state densities in considered cases, and they can be simply generalized for the situation (see Figure 3.10) when the state densities are arbitrary. In the general case for stimulated transitions between two bands of the quasicontinuum, the ratio of the upward-transition rate $W$ (lower $\rightarrow$ upper) to the downward transition one $W$ (upper $\rightarrow$ lower) must be equal to the ratio $\rho_{\text {upper }} / \rho_{\text {lower }}$ between the densities of states in the bands. Thus, one comes to the following equations for populations:

$$
\begin{aligned}
& \frac{d z_{\text {lower }}}{d t}=-\sigma P\left(z_{\text {lower }}-\frac{\rho_{\text {lower }}}{\rho_{\text {upper }}} z_{\text {upper }}\right) \\
& \frac{d z_{\text {upper }}}{d t}=\sigma P\left(z_{\text {lower }}-\frac{\rho_{\text {lower }}}{\rho_{\text {upper }}} z_{\text {upper }}\right),
\end{aligned}
$$

where $\sigma$ means the absorption cross-section from any level of the lower band, with the above reservation on the possibility of averaging this value. 
We may now write out the kinetic equations for subsequent stimulated transitions (see Figure 3.11) between many sectors of the quasicontinuum one quantum of radiation apart. We shall mark the total populations $z_{n}$ of the vicinities of narrow resonances as well as the corresponding densities of states $\rho_{n}$ by the index $n$. The equations take the form

$$
\begin{aligned}
\frac{d z_{n}}{d t}=\sigma_{n-1, n} P\left(z_{n-1}\right. & \left.-\frac{\rho_{n}-1}{\rho_{n}} z_{n}\right) \\
& -\sigma_{n, n+1} P\left(z_{n}-\frac{\rho_{n}}{\rho_{n+1}} z_{n+1}\right)
\end{aligned}
$$

These equations describe the dynamics of populations for given initial conditions and, for insight into the features of appearing distributions, one may test various explicit dependencies of the cross-section on the number

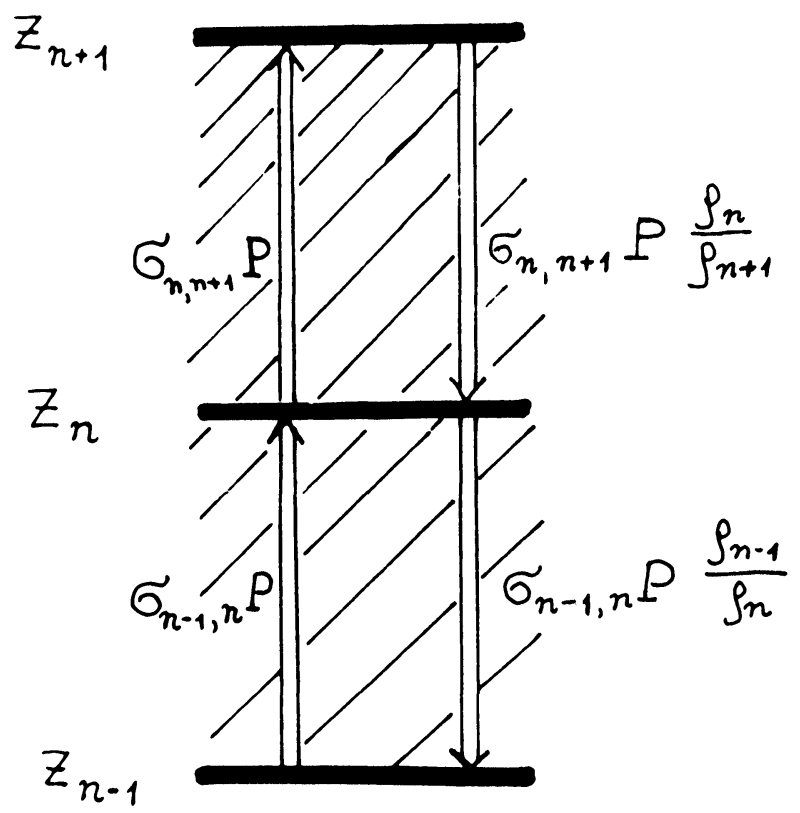

FIGURE 3.11 Multistep transitions between narrow resonant sectors in the vibrational quasicontinuum. 
of transitions which, of course, are of physical sense. It should be noted right away that one can distinctly see the analogy of Eq. (3.101) to those which describe the problems of one-dimensional random walk in the probability theory (see, for example, Ref. 199). A standard approach to studying such problems is the approximate procedure which comes to replacing the set of ordinary differential equations by one partial differential equation which is usually called the Focker-Planck equation.

If in Eq. (3.101) the rates of upward and downward transitions were equal we would actually deal with the well-known process of one-dimensional diffusion. If initially the distribution is concentrated near the boundary corresponding, in our case for example, to the initial conditions

$$
z_{0}(t=0)=1, \quad z_{n}(t=0)=0 \text { for } n \geqslant 1
$$

then the diffusion leads to the qualitative shape of distributions shown in Figure 3.12a. The maximum of distribution always falls on the boundary and, for that particular case when the diffusivity (or the transition rate for

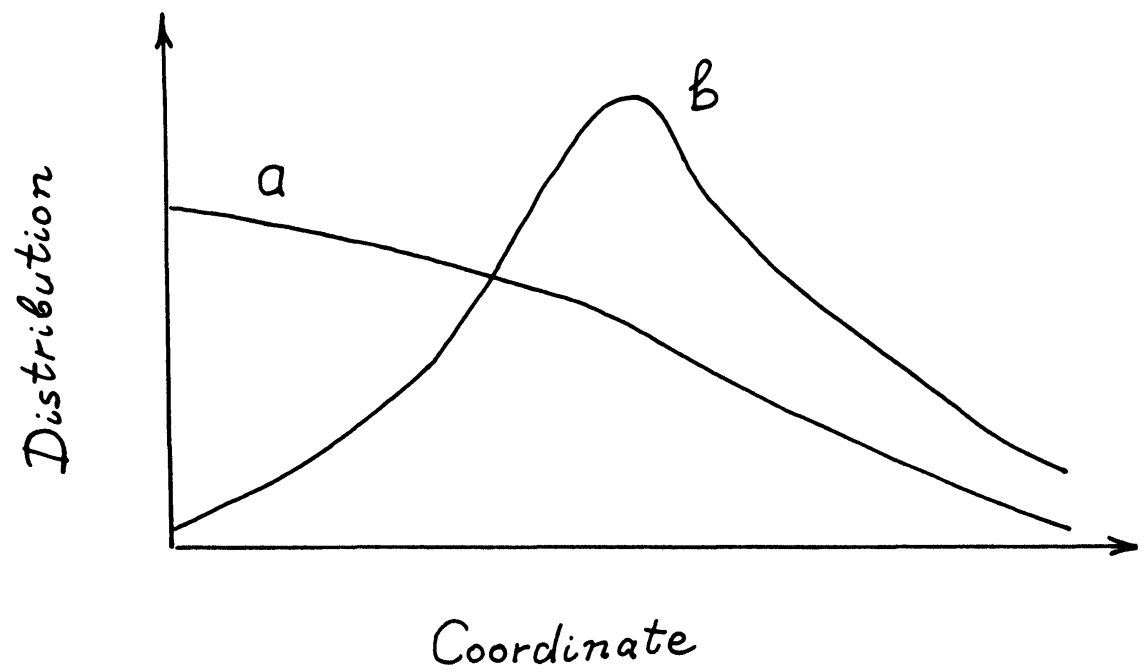

FIGURE 3.12 The qualitative shapes of distributions realized during processes like diffusion when initially the distribution concentrates near the boundary: (a) diffusion without drift; (b) diffusion plus drift. 
us) is a constant value, the width of distribution grows proportionally to $t^{1 / 2}$.

However, in Eq. (3.101) the rates of upward transitions are greater than those of downward ones since the density of molecular states increases versus the vibrational energy. Therefore, we may say that we soon deal with a process which can be referred to as diffusion plus drift. In this case, the qualitative shape of distribution is different, since the drift forces the maximum to be shifted from the boundary (see Figure 3.12b).

\subsubsection{Excitation for the case of constant cross-sections}

The difference in rates of upward and downward transitions is an important principal point, and we can illustrate this through direct calculations suggesting, for example, that in Eq. (3.101)

$$
\sigma_{n-1, n} \equiv \sigma=\text { const }, \quad \rho_{n-1} / \rho_{n} \equiv \alpha=\text { const }<1
$$

To simplify the mathematics let us consider the infinite number of bands, and Eq. (3.101), with the cross-sections and state densities chosen, take the form

$$
\begin{aligned}
& \frac{d z_{0}}{d t}=-\sigma P\left(z_{0}-\alpha z_{1}\right) \\
& \frac{d z_{n}}{d t}=\sigma P\left[z_{n}-1-(1+\alpha) z_{n}+\alpha z_{n}+1\right], 1 \leqslant n<\infty
\end{aligned}
$$

The explicit solution of Eqs. (3.104) with the initial conditions (3.102) is expressed through the infinite series of modified Bessel functions:

$$
\begin{aligned}
& z_{n}(t)=\alpha^{-n / 2} \exp [-(1+\alpha) \sigma P t]\left\{I_{n}\left(2 \alpha^{1 / 2} \sigma P t\right)\right. \\
& +\alpha^{1 / 2} I_{n}+{ }_{1}\left(2 \alpha^{1 / 2} \sigma P t\right)-(1-\alpha) \sum_{m=0}^{\infty} \alpha^{m / 2} I_{n}+2+m\left(2 \alpha^{1 / 2} \sigma P t\right)
\end{aligned}
$$

The modified Bessel functions can be presented as the series

$$
I_{n}(x)=\sum_{k=0}^{\infty} \frac{(x / 2)^{n}+2 k}{k !(n+k) !}
$$


and, using the elementary relations (see, for example, Ref. 200)

$$
2 \frac{d I_{n}}{d x}=I_{n-1}(x)+I_{n+1}(x), \frac{d I_{0}}{d x}=I_{1}(x),
$$

one may convince himself through the direct substitution that Eq. (3.105) actually gives the solution required. Though the solution (3.105) looks rather cumbersome, it is easy to estimate such very important characteristics of distribution as the mean $\langle n\rangle$ and the relative half-width $d=\left(\left\langle n^{2}\right\rangle\right.$ $\left./\langle n\rangle^{2}-1\right)^{1 / 2}$ [compare with Eq. (2.98) from Ref. 175]. The equation for $\langle n\rangle$ can be found if one multiplies Eqs. (3.104) by $n$ and carries out the summation over $n$ using the trivial equality

$$
\sum_{n=0}^{\infty} z_{n}(t)=1
$$

This equation has the form

$$
\frac{d\langle n\rangle}{d t}=\frac{d}{d t} \sum_{n=1}^{\infty} n z_{n}=(1-\alpha) \sigma P+\alpha \sigma P z_{0}
$$

hence it follows that

$$
\langle n\rangle=(1-\alpha) \sigma P t+\alpha \sigma P \int_{0}^{t} z_{0}(\tau) d \tau
$$

Right away one may see a principal difference between the cases when $\alpha=1$ and $\alpha<1$. With $\alpha=1$, the first linear term in Eq. (3.110) vanishes, and using Eq. (3.105) we get

$$
\langle n\rangle=\sigma P \int_{0}^{t} \exp (-2 \sigma P \tau)\left[I_{0}(2 \sigma P \tau)+I_{1}(2 \sigma P \tau)\right] d \tau
$$

At large values of the variable the modified Bessel functions entering Eq. (3.111) come to the following asymptotic form (see, for example, Ref. 200): 


$$
I_{0}(x) \simeq I_{1}(x) \simeq \frac{e^{x}}{(2 \pi x)^{1 / 2}}(\text { when } x \gg 1)
$$

Thus, if we are interested in the asymptotic behavior of the dependence of $\langle n\rangle$ on time with $\langle n\rangle \gg 1$, we have

$$
\langle n\rangle \simeq 2\left(\frac{\sigma P t}{\pi}\right)^{1 / 2}
$$

as it should be observed at the ordinary diffusion process. The case is somewhat different when $\alpha<1$. Then the integral entering Eq. (3.110) tends with $t \rightarrow \infty$ to a finite value. The integral of $z_{0}(t)$ taken over the whole infinite semiaxis is reduced to a sum of tabulated integrals and, as a result, one obtains the following asymptotes for $\langle n\rangle$ :

$$
\langle n\rangle \simeq(1-\alpha) \sigma P t+\frac{\alpha}{1-\alpha}
$$

which holds true provided, of course, that

$$
\sigma P t \gg \frac{\alpha}{(1-\alpha)^{2}}
$$

Let us estimate now the relative half-width of the distribution (3.105). To do this one may derive the equation for the mean-square $\left\langle n^{2}\right\rangle$, proceeding directly from Eqs. (3.104). This equation has the form

$$
\begin{aligned}
\frac{d}{d t}\left\langle n^{2}\right\rangle=\frac{d}{d t} \sum_{n} & =1 \\
& =2(1-\alpha) \sigma P\langle n\rangle+(1+\alpha) \sigma P+\alpha \sigma P z_{0}
\end{aligned}
$$

Using the above-obtained asymptotic expression for $\langle n\rangle$, we have the following asymptotes for the relative half-width of distribution:

$$
\begin{aligned}
d=\left(\frac{\left\langle n^{2}\right\rangle}{\langle n\rangle^{2}}-1\right)^{1 / 2} & \simeq \frac{1}{1-\alpha}\left(\frac{1+\alpha}{\sigma P t}\right)^{1 / 2} \\
& \simeq\left[\frac{1+\alpha}{(1-\alpha)\langle n\rangle}\right]^{1 / 2},
\end{aligned}
$$


from which it follows that, with the condition (3.115) fulfilled, $d \ll 1$, i.e., the distribution is concentrated near its mean. It also follows from Eq. (3.117) that the relative width of distribution increases as $\alpha$ approaches unity. The distributions shown in Figure 3.13 which are calculated with the use of exact formulas (3.105) illustrate well the found asymptotic estimates. These distributions are given for various values of $\alpha$, but for the common $\langle n\rangle=40$. It should be noted that, for $\alpha=0$, i.e., when the rate of upward transitions is negligible compared to that of downward ones, the distribution (3.105) comes to the Poisson distribution

$$
z_{n}=\frac{(\sigma P t)^{n}}{n !} \exp (-\sigma P t)
$$

Let us now discuss to what extent the picture just considered really reflects the excitation of the vibrational quasicontinuum of molecules. The state densities entering the general equations are associated with the sectors of the vibrational spectrum which are one quantum of radiation apart. If, for estimates, one neglects the energy of the ground state in the semi-

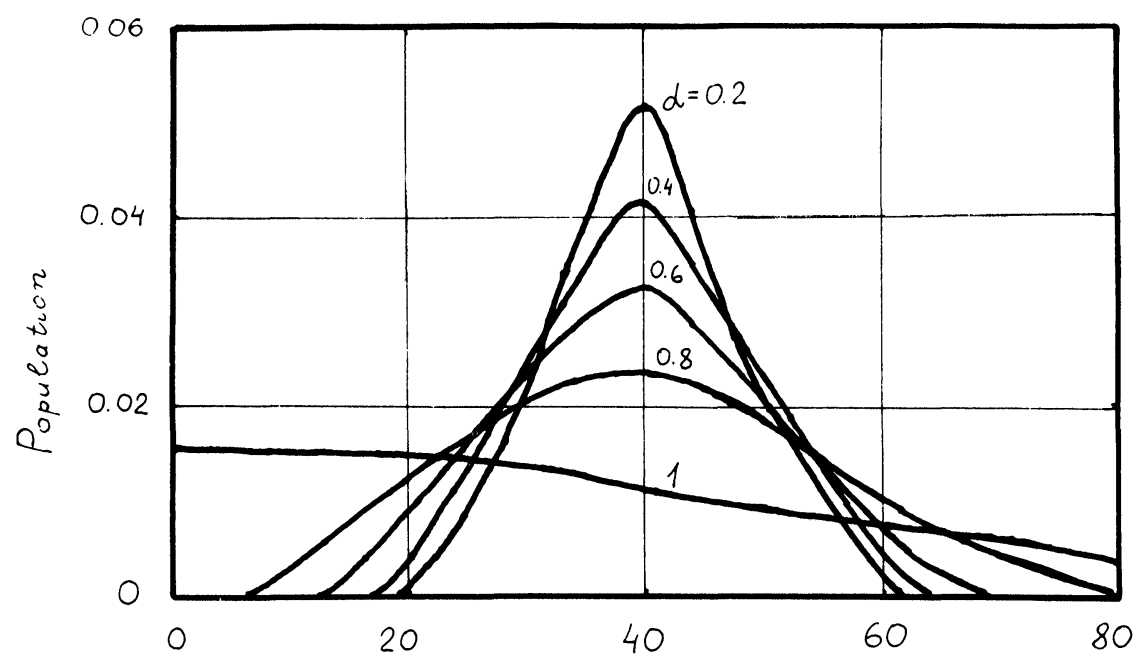

FIGURE 3.13 The distributions (3.105) for different values of the parameter $\alpha$ with the mean energy $\langle n\rangle=40$. 
classical expression (2.70) from Ref. 175, then the ratio of state densities of two adjacent bands with the energies $E_{\mathrm{vib}}$ and $E_{\mathrm{vib}}+\hbar \Omega$ is

$$
\frac{\rho\left(E_{\mathrm{vib}}\right)}{\rho\left(E_{\mathrm{vib}}+\hbar \Omega\right)} \sim\left(1+\frac{\hbar \Omega}{E_{\mathrm{vib}}}\right)^{-(s-1)}
$$

Taking the radiation frequency $1000 \mathrm{~cm}^{-1}$ one finds that, for example, for a seven-atom molecule, the ratio (3.119) does not exceed 0.6 even up to $E_{\mathrm{vib}} \simeq 40000 \mathrm{~cm}^{-1}$ which is somewhat higher than the typical dissociation limits. On the other hand, with $\alpha=0.6$ and $\langle n\rangle=40$, one finds from Eq. (3.117) that $d \simeq 0.15$. Thus, we should really expect the distribution produced by radiation to have a distinct maximum and be concentrated near its mean.

\subsubsection{Excitation for the case of varying cross-sections}

Another important point is how the real energy dependence of the crosssection of transitions in vibrational quasicontinuum affects the form of distribution. In Section 2.3.4 from Ref. 175 we have already discussed the qualitative shape of absorption bands in the vibrational quasicontinuum. As the energy increases, the maximum of absorption band is shifted by anharmonicity to the long-wave region, and the band itself is broadened. These two points cause the dependencies of the cross section on the number of transition to be different, in general, for different frequencies of radiation used. To obtain some simple estimates let us first ignore any changes in the band shape. This assumption, however, does not mean that the transition cross-section remains constant. Indeed, in our previous paper (Ref. 175) we have derived Eq. (2.81) which enables us to calculate the square of the dipole moment of transitions from a fixed $E_{\text {vib }}$-energy state integrated over the whole band and this integrated value, of course, grows versus the energy. It is logical that just this dependence is tested if one neglects changes in the band shape, and it will be called below the integral-crosssection law, for short.

For our purposes, it will be convenient here to transform Eq. (2.81) from Ref. 175 to another form. We consider for simplicity the energy of the lowest band to coincide with that of the ground state. Then one can express the cross-sections of all subsequent transitions through the crosssection $\sigma_{01}$. Indeed, for the cross-sections of any two successive transitions, one finds using Eqs. (2.81) from Ref. 175 the following equations: 


$$
\begin{aligned}
& \rho_{n-1} \sigma_{n-1, n}=\sigma_{01} \sum_{k=0}^{n-1}(k+1) \rho^{\prime}[(n-1-k) \hbar \Omega] \\
& \rho_{n} \sigma_{n, n+1}=\sigma_{01} \sum_{k=0}^{n}(k+1) \rho^{\prime}[(n-k) \hbar \Omega],
\end{aligned}
$$

where the equality of the field frequency $\Omega$ and that $\nu_{\mathrm{ir}}$ of the resonant mode is taken, and $\rho^{\prime}$ denotes the densities of states constructed by the rest of modes. Substracting the first of Eqs. (3.120) from the second one, we get

$$
\begin{gathered}
\rho_{n} \sigma_{n, n+1}-\rho_{n}-{ }_{1} \sigma_{n}-1, n=\sigma_{01} \sum_{k=0}^{n} \rho^{\prime}[(n-k) \hbar \Omega]=\rho_{n} \sigma_{01}, \\
\quad \text { or } \quad \sigma_{n, n+1}=\sigma_{01}+\frac{\rho_{n}-1}{\rho_{n}} \sigma_{n-1, n}
\end{gathered}
$$

Hence it is easy to obtain

$$
\sigma_{n, n+1}=\sigma_{01} \frac{1}{\rho_{n}} \sum_{k=0}^{n} \rho_{k},
$$

and, on substituting (3.122) into Eq. (3.101), we come to

$$
\begin{aligned}
\frac{d z_{n}}{d t} & =\sigma_{01} P\left\{\frac{z_{n}-1}{\rho_{n}-1} \sum_{k=0}^{n} \rho_{k}-\frac{z_{n}}{\rho_{n}} \sum_{k=0}^{n-1} \rho_{k}-\frac{z_{n}}{\rho_{n}} \sum_{k=0}^{n} \rho_{k}\right. \\
& \left.+\frac{z_{n}+1}{\rho_{n}+1} \sum_{k=0}^{n} \rho_{k}\right\}
\end{aligned}
$$

It only remains to include explicitly the dependence of the state density on the number of band. But first let us clear up some common features of solutions of Eq. (3.123) concerned with the mean energy and the relative half-width of distribution. For the mean one may derive directly from Eq. (3.123), multiplying by $n$ and summing, the following absolutely exact result:

$$
\begin{aligned}
& \frac{d\langle n\rangle}{d t}=\frac{d}{d t} \sum_{n=1}^{\infty} n z_{n}=\sigma_{01} P, \\
& \text { or }\langle n\rangle=\sigma_{01} P t
\end{aligned}
$$


Thus, the mean energy increases proportionally to time. In the same manner one may find the equation for the mean-square $\left\langle n^{2}\right\rangle$ which enters the expression for the relative half-width of distribution. This equation is

$$
\begin{aligned}
\frac{d\left\langle n^{2}\right\rangle}{d t}=\frac{d}{d t} \sum_{n=1}^{\infty} n^{2} z_{n} \\
\quad=\sigma_{01} P\left(2\langle n\rangle-1+2 \sum_{n=0}^{\infty} \frac{z_{n}}{\rho_{n}} \sum_{k=0}^{n} \rho_{k}\right),
\end{aligned}
$$

and one can see that it already includes the law according to which the density of states grows from band to band.

In Section 2.3.1 from Ref. 175 we discussed various approximations for describing the dependence of the density of molecular states on the vibrational energy. Suggesting that the features of distribution under strong excitation are of interest, i.e., when the terms with $n \gg 1$ play the dominant role in the sum in Eq. (3.125), we take for estimates the semiclassical approximation [see Eq. (2.70) from Ref. 175] where the energy of the ground state is neglected. As a result, considering only the two main terms of the asymptotic expansion, we have

$$
\frac{1}{\rho_{n}} \sum_{k=0}^{n} \rho_{k} \simeq \frac{1}{\mathrm{n}^{s}-1} \sum_{k=0}^{n} k^{s-1} \sim \frac{n}{s}+\frac{1}{2}
$$

and, on substituting this approximate relation into Eq. (3.125) and using Eq. (3.124), one finds that

$$
\left\langle n^{2}\right\rangle \sim\left(\sigma_{01} P t\right)^{2}\left(1+\frac{1}{s}\right)
$$

hence the relative half-width of distribution is

$$
d=\left(\frac{\left\langle n^{2}\right\rangle}{\langle n\rangle^{2}}-1\right)^{1 / 2} \sim s^{-1 / 2}
$$

Recall now that this same value had been already obtained when in Section 2.5.1 from Ref. 175 we discussed the properties of the vibrational Boltzmann distribution at high temperature [see Eq. (2.90) from Ref. 175].

Thus we may conclude that the Boltzmann distribution is a natural 
reference one to be compared with the real distribution produced by radiation in the vibrational quasicontinuum. In addition, we want to point out one specific case when Eq. (3.123) can be solved analytically, and the solution really coincides with the Boltzmann distribution. This is the case when the state densities are approximated by Eq. (2.68) from Ref. 175, i.e., when a molecule is treated as a degenerate $s$-dimensional harmonic oscillator. We do not write out the equations and their solution for this case to give the reader a chance to practice on his own and convince himself of the results. We should recall, however, that the representation of the general Eqs. (3.102) in the form (3.123) implies that the crosssection of successive transitions in quasicontinuum evolves in exact accordance with the integral-cross-section law. In the general case, the change in the band shape as the vibrational energy grows in what has been discussed in Ref. 175 (Section 2.3.4) leads, of course, to a different cross-section law. Here the situation is probably the most realistic when the radiation frequency lies near the center of the band at the lower transitions. In this case, because of the anharmonic shift of the band to the long-wave spectral region, the growth of the real cross-section is slower than that of the integral one (in particular, the cross-section may even decrease), and one can understand that the mean energy must grow slower than linearly and the distribution itself must be narrower than the Boltzmann one. Otherwise, if at the lower transitions the radiation frequency lies at the long-wave tail of the band, then the real cross-section grows more rapidly up to a certain energy than the integral one, and the distribution is perhaps wider than the Boltzmann one.

\subsubsection{Influence of dissociative decay and rotational structure}

Below, in this series of papers, we shall discuss specific numerical calculations of the dynamics of excitation of molecules in the vibrational quasicontinuum which will be compared with the actual experiments. As a rule, we shall find support for the rough qualitative picture presented here. More detailed calculations must involve two more points which are not hard to include into the calculation procedure.

First, as far as the states above the dissociation limit are concerned, the terms which describe the decay of a molecule into fragments must enter the equations. Therefore, a more general form of kinetic equations for the quasicontinuum dynamics is 


$$
\begin{aligned}
\frac{d z_{n}}{d t}=\sigma_{n}-{ }_{1, n} P( & \left.z_{n}-1-\frac{\rho_{n}-1}{\rho_{n}} z_{n}\right) \\
& \quad-\sigma_{n, n}+{ }_{1} P\left(z_{n}-\frac{\rho_{n}}{\rho_{n}+1} z_{n}+1\right)-k_{n} z_{n},
\end{aligned}
$$

where $k_{n}=0$ for the states below the dissociation limit and the decay rates $k_{n}$ for the states above the dissociation limit can be calculated with the use of the RRKM theory discussed in Section 2.4 from Ref. 175.

Second, so far we have not allowed for rotations. The shape of the absorption contour in the quasicontinuum, with transitions in various rotational branches involved, has been already discussed in Ref. 175 (Section 2.3.4). For example, for spherical tops as well as for parallel bands of symmetrical tops, the transitions are possible in three branches from any vibration-rotation level with the rotational quantum number $J$. With the cross-sections of these transitions being designated as $\sigma_{n, n}^{J, J} \subsetneq{ }_{1}^{1}, \sigma_{n, n}^{J, J}+1$, and $\sigma_{n, n}^{J, J}+{ }_{1}^{1}$, one should write the general equations for populations which allow for the change in $J$ in the following form:

$$
\begin{aligned}
& \frac{d z_{n, J}}{d t}=\sigma_{n}^{J}={ }_{1, n}^{1, J} P\left(z_{n}-1, J-1-\frac{\rho_{n}-1}{\rho_{n}} z_{n, J}\right)+\sigma_{n}^{J, J}-{ }_{1, n} P\left(z_{n-1, J}\right. \\
& \left.-\frac{\rho_{n}-1}{\rho_{n}} z_{n, J}\right)+\sigma_{n}^{J} \pm{ }_{1, n}^{1, J} P\left(z_{n}-1, J+1-\frac{\rho_{n}-1}{\rho_{n}} z_{n, J}\right) \\
& -\sigma_{n, n}^{J, J} \mp{ }_{1}^{1} P\left(z_{n, J}-\frac{\rho_{n}}{\rho_{n}+1} z_{n}+1, J-1\right)-\sigma_{n, n}^{J, J}+{ }_{1} P\left(z_{n, J}\right. \\
& \left.-\frac{\rho_{n}}{\rho_{n}+1} z_{n}+1, J\right)-\sigma_{n, n}^{J, J}+{ }_{1} P\left(z_{n, J}-\frac{\rho_{n}}{\rho_{n+1}} z_{n+1, J}+1\right)
\end{aligned}
$$

As before, the populations are averaged over the resonant vicinities of bands and now the notation $z_{n, J}$ refers not only to the $n$-th band but also to the rotational quantum number $J$.

\subsubsection{Multiphoton transitions into quasicontinuum}

So far, we have treated various fragments of a complex picture of the molecular spectrum exhibiting elementary one-photon and multiphoton transitions between the lower quantum levels as well as successive ones 


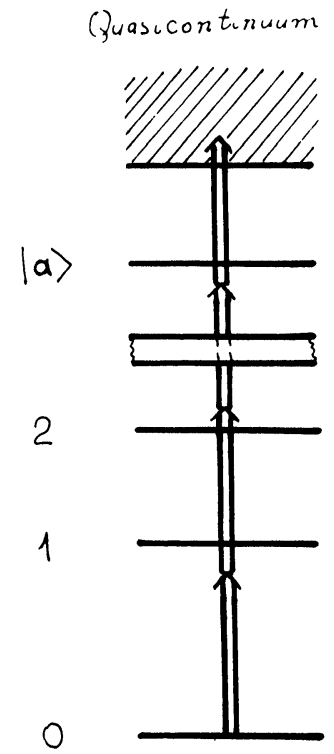

FIGURE 3.14 The diagram of levels showing a possibility for direct multiphoton transitions into the quasicontinuum.

within the quasicontinuous spectrum of high vibrational states. We have repeatedly laid emphasis on the intricacy for insight into the problem of the behavior of a molecule in the radiation field on the whole. In this short section we wish to add one more simple fragment. Let us conventionally divide the energy spectrum of a molecule into two regions (see Figure 3.14): the lower region consisting of several discrete levels, the transitions between which are quasiresonant with respect to the field frequency, and the upper one where the transition spectrum is quasicontinuous. In the case when the Rabi frequencies in the lower-level subsystem exceed the characteristic value of detunings from resonances, the upper level of the lower subsystem (designated as $|a\rangle$ ) is excited effectively enough to ensure further excitation to the quasicontinuum. Let, however, the Rabi frequencies be much smaller than the characteristic detuning, including the multiphoton ones. Then the population of the level $|a\rangle$ is much less than unity, i.e., $\rho_{a a} \ll 1$. This value can be calculated within perturbation theory and if, for example, the lower subsystem consists just of two levels, then 


$$
\rho_{a a} \simeq \frac{\gamma_{0 a}^{2}}{\left(\Omega-\omega_{a 0}\right)^{2}} .
$$

If the lower subsystem consists of three levels, then

$$
\rho_{a a} \simeq \frac{\gamma_{01}^{2} \gamma_{1 a}^{2}}{\left(\Omega-\omega_{10}\right)^{2}\left(2 \Omega-\omega_{10}-\omega_{a 1}\right)^{2}},
$$

etc. But, despite the inequality $\rho_{a a} \ll 1$, the excitation efficiency may be considerable if many irreversible decays of the level $|a\rangle$ into the quasicontinuum have time to occur during the radiation pulse. The molecule as if leaks from the ground state into the quasicontinuum. ${ }^{201}$ The leakage rate is given by the following rather evident equation:

$$
W=\rho_{a a} \sigma_{a} P,
$$

where $\sigma_{a}$ is the cross-section of the transition from the level $|a\rangle$ to the quasicontinuum. Physically this effect is rather clear and, to some extent, is identical to the well-known quantum-mechanical tunneling through a barrier but its treatment in terms of the direct multiphoton transition into the quasicontinuous spectrum is perhaps more essentially accurate.

In Section 3.3 we considered the dynamics of multiphoton transitions between the discrete states. Here, on the contrary, the upper state is a band of levels. If they are spaced closely enough then, like the one-photon process (see Section 3.7), the multiphoton decay is also described by a simple kinetic equation but in this case one must use the multiphoton Rabi frequency instead of the one-photon Rabi frequency. Thus, the rate of multiphoton transition into the quasicontinuum must be proportional to $I^{n}$, where $n$ is the number of radiation quanta required for the transition. Using Eq. (3.53) for the multiphoton Rabi frequency, one can derive the following expression for this rate:

$$
\begin{gathered}
W_{n}^{(n)}= \\
\left|\frac{\mu_{01} \mu_{12} \cdot \ldots \cdot \mu_{n-2, n-1}}{\left(\Omega-\omega_{10}\right)\left(2 \Omega-\omega_{10}-\omega_{21}\right) \cdot \ldots \cdot\left[(n-1) \Omega-\omega_{10}-\omega_{21}-\ldots-\omega_{n-1, n-2}\right]}\right|^{2} \\
\left(\frac{2 \pi}{\hbar^{2} c}\right)^{n-1} \sigma_{n-1, n} I_{n}
\end{gathered}
$$


If there are several channels one should, of course, sum over them in the manner discussed in Section 3.3.

Some specific estimates will be given in Section 5 of our series of papers, when discussing various theoretical approaches to the description of excitation of the lower molecular vibrational levels by the laser field. Here we want just to note that among all treated resonant processes, the multiphoton transition into the quasicontinuum seems to be the only rather universal one, since it does not require accidental coincidence of the field frequency with that of any transition. But it is quite obvious from the estimates on multiphoton Rabi frequencies obtained in Section 3.4 that this process is probably effective in that case only when the limit of vibrational quasicontinuum is low in energy.

\subsection{Concluding remarks}

In this chapter we have treated some questions on interaction of multilevel molecular systems with radiation. We have tried to embrace the most principal points such as the resonant criteria for one-photon and multiphoton processes, various approximations for describing the action of radiation on molecules, and the role of some relaxation processes. As will be seen from our further discussions, some of the experimental observations do not need anything more explained, but some important results cannot be interpreted within the simple schemes. More complex theoretical treatments are called for. We shall examine them, but do not wonder if you do not find in this series of papers a comprehensive explanation for all the experiments. And all this despite the work of many experts in the theory! To make the theory more complex is not always the best and most universal way for insight into the problem. By using the estimates obtained in this section and correlating them with experiment you will be able yourself to detect the theoretical points that are open to criticism. Experiment is of course the chief judge, and we believe that some of our readers will be able to find key experimental ideas, the theoretical treatment of which will allow us in the future to replace the modest "elements of theory" in the title of this chapter by exhaustive "theory". 


\section{SECTION 4. PHYSICS OF INTERACTION OF A POLYATOMIC MOLECULE WITH AN INTENSE IR FIELD}

This Section gives a short phenomenological description of the basic effects arising from multiphoton excitation of a polyatomic molecule. These effects are described in more detail in Sections 5 to 7. The qualitative description of MP excitation and dissociation of polyatomic molecules presented here is of particular use for a reader who is interested in the applications of this effect described in the last Sections, 8 and 9. One can turn to these Sections, omitting the comprehensive discussion of all the details of the effect in Sections 5 to 7 .

\subsection{Main stages of MP excitation of polyatomic molecules by IR radiation}

The introduction (Section 1.2) of the first article of this series ${ }^{202}$ presented the simplest model of multiphoton excitation and dissociation of polyatomic molecules in an intense IR field. According to this model (Figure 1.3 in Ref. 202) a polyatomic molecule, as the store of its vibrational energy $E_{\mathrm{vib}}$ increases, passes in succession through different energy regions characterized by different densities and width(s) of vibrational-rotational levels:

$\begin{array}{lll}\begin{array}{l}\text { discrete } \\ \text { vibrational } \rightarrow\end{array} & \begin{array}{l}\text { quasicontinuum } \\ \text { of vibrational } \rightarrow \\ \text { levels }\end{array} & \begin{array}{l}\text { real } \\ \text { levels } \\ \text { of vibrational levels }\end{array} \\ \text { with } E_{\mathrm{vib}}<E_{\mathrm{qc}} & \text { with } E_{\mathrm{qc}}<E_{\mathrm{vib}}<D & \text { with } E_{\mathrm{vib}}>D \\ \text { (region I) } & \text { (region III) }\end{array}$

where $E_{\mathrm{qc}}$ is the lower limit of the vibrational quasicontinuum, or in other words, the stochastization limit of vibrational energy, and $D$ is the lower dissociation limit. In Section 2.3 (article I, Ref. 202) consideration was given to the meaning of a quasicontinuum of vibrational energy and its lower limit $E_{\mathrm{qc}}$ for polyatomic molecules.

The interaction of a polyatomic molecule with an IR field essentially depends on which of the vibrational energy regions previously mentioned it is located in. We are going to discuss it briefly on the basis of the data given in Section 2 (article I, Ref. 202) and Section 3 of the present article. 


\subsubsection{Role of IR radiation intensity in excitation of lower vibrational levels}

In the region of lower discrete vibrational-rotational levels (region I) a molecule acted upon by an IR field undergoes successive stimulated resonant transitions on the ladder of vibrational levels. Of particular importance here is that each vibrational level splits into a great number of rotational and vibrational-rotational sublevels due to a different type of vibration-rotation interaction (see Section 2.1.5 in Ref. 202). On the other hand, because of this the molecules are distributed initially over a lot of sublevels even if they are concentrated in the ground vibrational state. This, of course, impedes simultaneous excitation of the molecules from all the sublevels under a monochromatic IR field. On the other hand, such splitting opens up many potential ways for multistep and multiphoton excitation of molecules in rather intense IR fields despite of anharmonic frequency shifts in successive vibrational upward transitions (see Section 2.1 in Ref. 202).

The IR radiation intensity essential for a molecule to pass through the entire region of lower vibrational levels up to the region of vibrational quasicontinuum depends substantially on the type of molecule. Indeed, the type of molecule governs the value of anharmonicity, the character of splitting of each vibrational level and the position of the vibrational quasicontinuum limit. For a three-atom molecule, for instance, the anharmonic shift is large, the number of split sublevels for each vibrational level is small and the vibrational quasicontinuum limit, one may say, is almost lifted to the dissociation limit $\left(E_{\mathrm{qc}} \simeq D\right)$. In this case extremely intense IR fields (see the calculations in Section 3.2) are required for multiphoton excitation of such molecules. Therefore, despite many attempts made in special experiments ${ }^{203-205}$ MP excitation and dissociation of three-atom and especially diatomic molecules has not been observed experimentally yet.

In another limiting case, on the contrary, for complex polyatomic molecules, the anharmonic shift is moderate, the number of split sublevels is very large and the vibrational quasicontinuum limit drops very low, down to the first vibrational level $\left(E_{\mathrm{qc}} \simeq \hbar \Omega\right)$. In this case it is possible to excite the molecules from all the ground states directly to the vibrational quasicontinuum by means of one-quantum transitions. Such excitation calls for IR radiation of very low intensity. The situation is typical, for example, in the case of isolated molecular ions with the number of atoms no less than 
9 , i.e., with the number of vibrational modes $S \geqslant 20$, which fall within the vibrational quasicontinuum as the radiation intensity is of the order of $1 \mathrm{~W} / \mathrm{cm}^{2} .206$

Most of the polyatomic molecules for which MP excitation is observed and studied lie intermediately between the above extremes. Unfortunately, it is impossible to give a universal description for the excitation of molecules in the region of lower vibrational levels (region I) since such excitation depends on the structure of vibrational-rotational levels of the particular molecule. Therefore, the excitation of molecules in region I has been so far based most likely on experiment rather than on calculations. This is due, of course, to the scant spectroscopic data on the molecules in lower vibrational states except the ground and first excited states. Despite this, it is possible to formulate certain general considerations on the MP excitation of polyatomic molecules at lower vibrational transitions.

For a considerable fraction of the molecules distributed initially over lower sublevels to be involved in effective MP excitation in region I and to reach the vibrational quasicontinuum (region II), it is necessary that the IR laser radiation should comply with at least two conditions: 1) the radiation frequency $\Omega$ must lie within the band of vibrational absorption; 2) the radiation intensity $P$ must exceed some value of $P(\mathrm{I})$

$$
P \gtrsim P(\mathrm{I})
$$

The value of $P(\mathrm{I})$ depends greatly on the type of molecule, the accuracy of tuning the IR field frequency $\Omega$ to the MP excitation resonance and the fraction of molecules which must be excited to the vibrational quasicontinuum limit. So, understandably, the value of the $P(I)$ parameter varies over a very wide range. In the case of simple light polyatomic molecules, for example, in region I, 5 or 6 IR photons should be absorbed if the $\Omega$ frequency is detuned considerably relative to the exact resonances of successive stimulated transitions $v=0 \rightarrow \ldots \rightarrow v=6$. In this case, as the calculations in the foregoing section show (Section 3.2.4), the value of $P(\mathrm{I})$ may be as high as $10^{7}$ to $10^{9} \mathrm{~W} / \mathrm{cm}^{2}$. On the contrary, for heavy complex molecules $P(\mathrm{I})$ is reduced to $10^{3}$ to $10^{5} \mathrm{~W} / \mathrm{cm}^{2}$. From such wide variation the $P(\mathrm{I})$ value is rather conventional. It is introduced here only to emphasize a critical parameter of MP excitation of molecules in the region of lower vibrational transitions irrespective of their subsequent MP excitation in the vibrational quasicontinuum. 


\subsubsection{Role of IR radiation energy fluence in excitation of vibrational quasicontinuum}

The molecules which have reached the vibrational quasicontinuum limit are able to go on absorbing the IR radiation. It is much simpler to describe the IR MP excitation in region II than in region I because it is a successive multistep absorption of IR photons. In this region there is no need for an intense field to compensate for appreciable detunings between the IR field frequency $\Omega$ and the vibrational transition frequency. Therefore, the absorption in region II is universal by nature and can be described in terms of the absorption cross-section $\sigma\left(\Omega, E_{\mathrm{vib}}\right)$ depending on the vibrational energy $E_{\text {vib }}$ (see Section 3.5).

In essence, the absorption of IR radiation by excited molecules in region II is reduced to vibrational heating of the whole of the molecule, as was pointed out by Bloembergen. ${ }^{207}$ For strong vibrational heating up to energies $E_{\text {vib }} \simeq D$ it is apparently necessary that absorption saturation at the transitions of vibrational quasicontinuum should be obtained. In the case of an isolated molecule which does not lose its energy, this means that the IR radiation fluence at the $\Omega$ frequency must satisfy the condition

$$
\Phi \geqslant \Phi_{\text {sat }}(\mathrm{II}) \simeq \frac{\hbar \Omega}{\sigma(\Omega)},
$$

where $\Phi_{\text {sat }}$ (II) is the energy fluence of a laser pulse saturating the transition absorption in the vibrational quasicontinuum.

In fact, in the case of intense MP excitation of molecules, when the number of absorbed IR photons $\langle n\rangle \simeq D / \hbar \Omega \gg 1$, a more rigid condition should be fulfilled. It follows from relations (3.118) or (3.124) given in the previous Section

$$
\Phi \geqslant \frac{\langle n\rangle \hbar \Omega}{\sigma(\Omega)}=\frac{D}{\sigma(\Omega)}=\Phi(\mathrm{II}),
$$

where $\Phi(\mathrm{II})$ is the laser pulse energy fluence essential for strong MP excitation of a molecule to the dissociation limit.

Thus, the excitation of a polyatomic molecule in region II depends not on the radiation intensity but only on energy fluence. The estimation presented is, of course, qualitative by nature since it does not allow for variations in the cross-section $\sigma\left(\Omega, E_{\mathrm{vib}}\right)$ as molecules are being excited 
(see Section 2.3.4 in Ref. 202 and below in Section 4.3). But it determines qualitatively correctly the critical parameter $\Phi($ II) of MP excitation of molecules in the region of the vibrational quasicontinuum.

Since the MP excitation of molecules in regions I and II is governed by different parameters of IR radiation, intensity $P$ and energy fluence $\Phi$, conditions (4.1) and (4.3) must be fulfilled at the same time to provide MP excitation of the molecule. This point is considered below in more detail.

Let a polyatomic molecule be exposed to IR radiation for the time $\tau_{p}$ that does not exceed the time of its isolation $\tau_{\text {isol }}$ or its collisions with other molecules, the walls and so on, that is

$$
\tau_{p} \lesssim \tau_{\text {isol }}
$$

Under condition (4.4) only collisionless photoexcitation of the molecule due to absorption of a large number of IR photons is possible.

For most of the experiments in low-pressure gases (below 1 Torr) $\tau_{\text {isol }}$ $\leqslant 10^{-6} \mathrm{~s}$ (see Section 2.5 in Ref. 202), and $\mathrm{CO}_{2}$ laser pulses with their duration $\tau_{p} \simeq 10^{-7} \mathrm{~s}$ are used for irradiation. In these cases the energy fluence essential for effective excitation of molecules to the vibrational quasicontinuum $\Phi>\Phi($ II) dictates that the pulse intensity be

$$
P=\Phi(\mathrm{II}) / \tau_{p} \gg P(\mathrm{I})
$$

And the typical values of $\Phi($ II) in that region lead to radiation intensities from $10^{6}$ to $10^{8} \mathrm{~W} / \mathrm{cm}^{2}$. At such intensity, condition (4.1) can be fulfilled easily, i.e., the MP excitation of molecules to region II is always possible. In other words, in experiments of this kind, the absorption in the vibrational quasicontinuum is the limiting process of MP excitation.

This manifests itself more vividly in experiments with shorter pulses $\tau_{p}$ $\simeq 10^{-9} \mathrm{~s}$ when, according to (4.5), higher intensities can be attained $\left(10^{8}\right.$ to $10^{10} \mathrm{~W} / \mathrm{cm}^{2}$ ). Such intensities ensure full excitation of molecules from all initial vibrational-rotational states to the region of vibrational quasicontinuum. Such experiments were performed in Ref. 208 especially to ensure MP excitation of the maximum (100\%) fraction of molecules.

Figure 4.1 shows the regions of laser radiation intensities and energy fluences which are essential for MP excitation of molecules according to conditions (4.1) and (4.3). In a simple way it also shows the shape of the vibrational energy distribution of a polyatomic molecule in different regions 


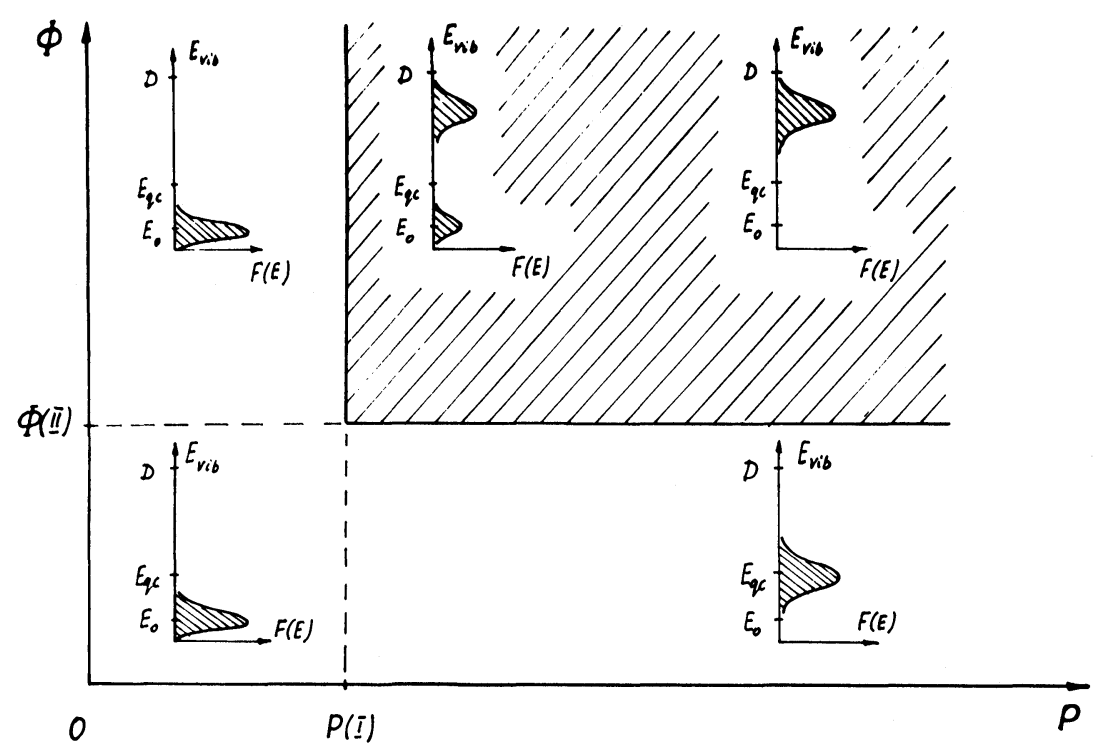

FIGURE 4.1 Intensities $P$ and energy fluences $\Phi$ of IR laser radiation essential for MP excitation of molecules to the dissociation limit. MP excited molecules lie in the shaded area. The vibrational distribution of molecules $F\left(E_{\text {vib }}\right)$ formed from MP absorption is shown in a simplified way for each of the regions of the parameters $(\Phi, P)$.

of the parameters $(\Phi, P)$. It may be seen that in the region $\Phi>\Phi(\mathrm{II})$ the increase of the $P$ intensity attained due to a reduction in pulse duration results in more efficient excitation of molecules to region II.

On the contrary, in experiments with long times $\tau_{\text {isol }}$, for example, in the above-mentioned experiments ${ }^{206}$ with molecular ions $\left(\tau_{\text {isol }} \geq 1 s\right)$, one can use very long pulses and even continuous radiation without violating condition (4.4). In this case the energy fluence critical for MP excitation of molecules in region II can be apparently realized at comparatively low intensities of IR radiation. The situation realized here is opposite to (4.5)

$$
\Phi(\mathrm{II}) / \tau_{p} \ll P(\mathrm{I})
$$

The limiting process here is the excitation of molecules at lower levels since the low intensity of IR field does not provide MP excitation of molecules in region II and, therefore, it becomes impossible to observe 
MP excitation. Exceptions to this are molecules with large numbers of degrees of freedom $s$ for which the lower limit of the vibrational quasicontinuum probably goes down to the level $v=1$. In this case there is no need for MP excitation of molecules in region I because right after resonant absorption of one IR photon the molecule enters region II. It is in the case of such polyatomic molecular ions that strong MP excitation and dissociation in a continuous IR field of low intensity has been observed. ${ }^{206}$

The region of MP excitation and dissociation of molecules (shaded) shown in Figure 4.1 is highly dependent of the type of a polyatomic molecule as has been already noted. In the case of comparatively simple molecules $\left(\mathrm{BCl}_{3}, \mathrm{C}_{2} \mathrm{H}_{4}\right.$, etc.) MP excitation necessitates both high intensities and high energy fluences since the values of $P(\mathrm{I})$ and $\Phi(\mathrm{II})$ for simple molecules are rather high (see the data from the next article III). From practical considerations such laser radiation can be easily produced in the form of short powerful pulses. And conversely, in the case of MP excitation of molecules with large numbers of atoms $\left(\mathrm{S}_{2} \mathrm{~F}_{10}, \mathrm{SF}_{5} \mathrm{NF}_{2}\right.$, etc. $)$ it is possible to use IR radiation of comparatively moderate intensity and lower energy fluence since the values of $P(\mathrm{I})$ and $\Phi(\mathrm{II})$ for such molecules are small (see the data in the next article III, in Sections 5 and 6). Such radiation parameters have been readily available, of course, for a long time, but their applications have been limited because it has been difficult to implement condition (4.4) of collisional excitation for a long time. In experiments with molecular gases at a pressure of about 1 Torr short pulses have to be used when condition (4.1) is exceeded.

\subsection{MP excitation of molecules at lower vibrational transitions}

In a sufficiently intense IR field a polyatomic molecule absorbs a considerable number of photons, passing in succession all the considered regions of vibrational energy. Such MP absorption by a multilevel molecular system features two specific behavior characteristics: 1) an even continuous increase of the number of absorbed IR photons with increasing radiation intensity; 2) a resonant dependence of MP absorption at varying radiation frequency. An important parameter is the fraction of molecules participating in MP absorption of IR radiation and caused to enter the region of the vibrational quasicontinuum. Let us consider briefly these most important characteristics of MP absorption. They will be discussed in more detail in Section 5 of the next paper III. 


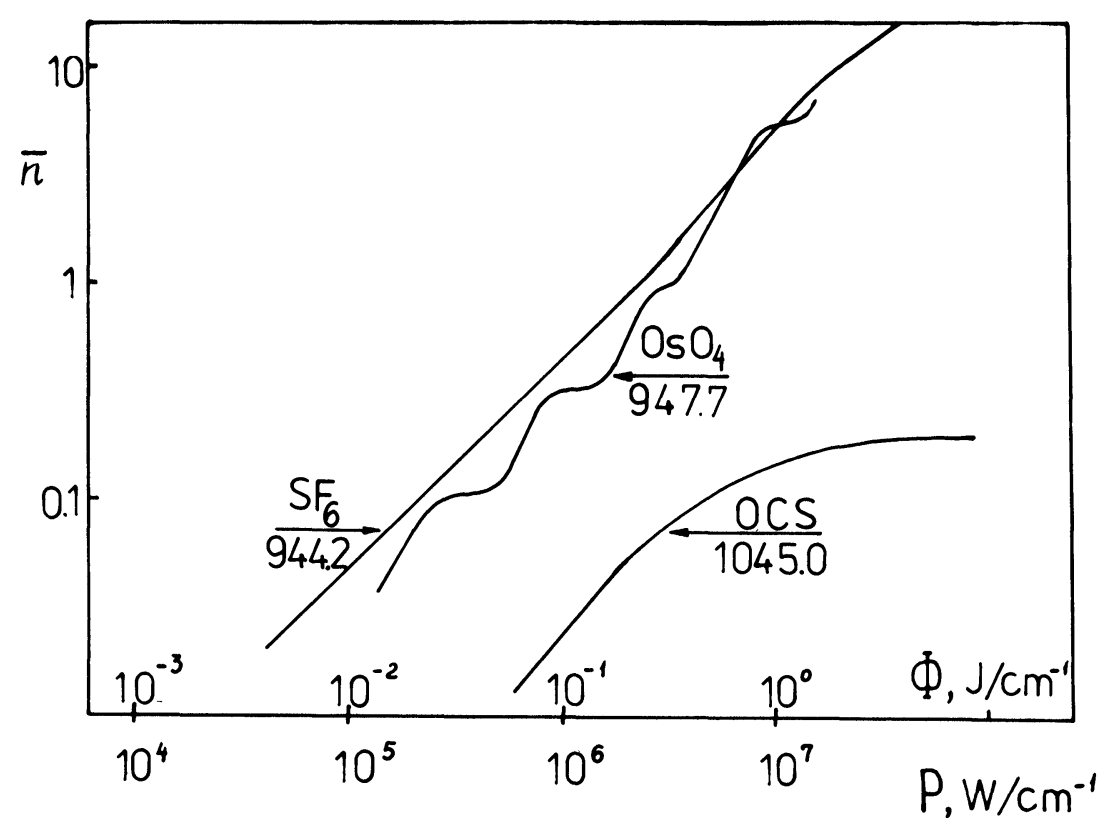

FIGURE 4.2 Dependences of the average number of IR photons absorbed per one molecule in the volume under irradiation for $\mathrm{SF}_{6}$ (from Ref. 203), $\mathrm{OsO}_{4}$ (from Ref. 210) and OCS (from Ref. 203). The values of the IR radiation frequency $\Omega$ are given in terms of $\mathrm{cm}^{-1}$.

\subsection{1. "Nonsaturable" behavior of MP absorption of IR radiation by polyatomic molecules}

This property of MP absorption of IR radiation was observed in the very first experiments. ${ }^{203,209}$ Figure 4.2 shows the experimental dependence of the average number of absorbed IR photons per $\mathrm{SF}_{6}$ molecule in the volume under irradiation* on the energy fluence $\Phi$ or radiation intensity $P$ (the pulse duration was constant, $\tau_{p}=90 \mathrm{~ns}$ ). As may be seen, this dependence

\footnotetext{
*Attention must be drawn to two different notations $(\langle n\rangle$ and $\bar{n})$ for the average number of absorbed IR photons which are fundamentally different. The $\langle n\rangle$ value introduced in Section 3 denotes the average level of vibrational excitation in the ensemble of excited molecules. The $\bar{n}$ value introduced here denotes the average number of IR photons absorbed per molecule in the volume under irradiation. These values may not coincide, first, if not all the molecules participate in MP absorption (in this case $\langle n\rangle \geqslant \bar{n}$ ) and, secondly, if the initial excitation level is $\left\langle n_{0}\right\rangle$ high enough, i.e., when $\left\langle n_{0}\right\rangle \gg 1$.
} 
is continuous, a little slower than a linear dependence. This points to the excitation of higher vibrational levels after the level $v=1$ or simultaneously with it, i.e., to a gradual increase of the average level of vibrational excitation with increasing intensity $P$ or energy fluence $\Phi$. MP absorption saturation sets in at $\bar{n} \gg 1$ because of dissociation of the excited molecules when they fall within region III. Similar dependences were obtained later for many other polyatomic molecules. They will be considered in more detail in Section 5 of the next paper III.

The even $\bar{n}(\Phi)$ dependences observed in the experiments seem at first to disagree with the sequence of two different stages of MP excitation presented in Section 4.1. Indeed, let the molecule be in any initial state. With small intensities (or small $\Phi$ ) the dependence of $\bar{n}$ must increase linearly until the transition $v=0 \rightarrow v=1$ is saturated. Then because of the absence of the exact resonance of the IR field frequency $\Omega$ with the successive vibrational transitions $v=0 \rightarrow v=1 \rightarrow \ldots v=E / \hbar \Omega$ MP absorption must be low up to the intensities $P \simeq P(\mathrm{I})$ which compensate for the frequency detuning of successive stimulated transitions. In other words, the $\bar{n}(P)$ dependence in the region $P<P(\mathrm{I})$ must be quite nonlinear as it is schematically shown in Figure 4.3 . With $P \gtrsim P(\mathrm{I})$ the energy growth of a molecule due to the transitions in the vibrational quasicontin-

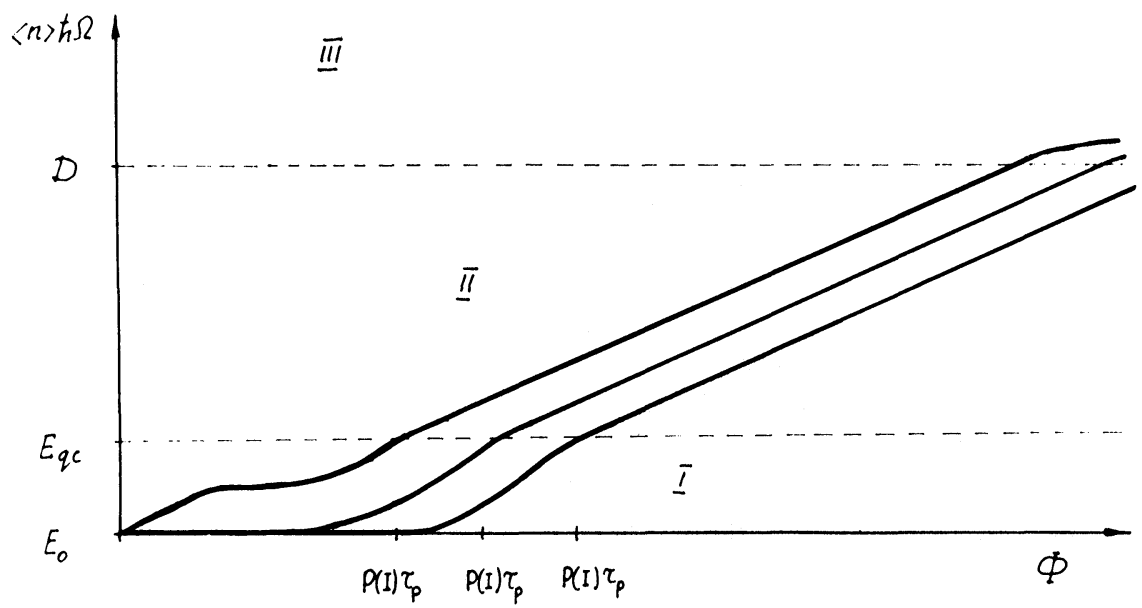

FIGURE 4.3 Simplified dependence of the excitation level on laser pulse energy fluence for molecules in different initial states having a different degree of resonance with the IR field in the region of lower vibrational transitions. 
uum must be linear by character since, according to the calculations in Section 3.5, $d\langle n\rangle / d \Phi=\sigma_{\mathrm{qc}}\left(\Omega_{1}\right)$.

Strictly speaking, the $\bar{n}(\Phi)$ dependence can actually be nonlinear due to the fact that the absorption cross-section $\sigma_{\mathrm{qc}}$ in region II depends on vibrational energy. More accurately the growth of vibrational energy of a molecule can be found from the equation

$$
\frac{d\langle n\rangle}{d \Phi}=\sigma_{\mathrm{qc}}(\Omega,\langle n\rangle)
$$

To determine its out-of-linearity in region II it is sufficient to know the $\sigma_{\mathrm{qc}}\left(\Omega, E_{\mathrm{vib}}\right)$ dependence. Continuous increase of the number of absorbed photons $\langle n\rangle$ must take place up to $\langle n\rangle \simeq D / \hbar \Omega$ when the dissociation of molecules in region III becomes essential.

As may be seen from Figure 4.2, no well-pronounced bend can be observed, in fact, in the experimental dependence for $\mathrm{SF}_{6}$. In other words, there is no material difference between the MP absorption at the lower vibrational transitions and in the vibrational quasicontinuum. This can be explained by the initial distribution of molecules over many rotational levels in the state $v=1$. Indeed, a small fraction of molecules being in an exact multistep resonance with the IR field has a low value for $P(\mathrm{I})$ and quickly falls within region II. Further vibrational excitation in region II, however, is possible just at sufficiently large values of $\Phi \geqslant \Phi_{\text {sat }}$ (II). For any other fraction of molecules the multistep resonance with the IR field in region I is not so exact and, therefore, considerable intensity is required for their MP excitation. Yet the MP excitation of all the molecules in region II comes about almost in the same way by (4.7). The observed smooth dependence $\bar{n}(\Phi)$ can result from summing up all such groups of molecules having a different degree of frequency detuning in region I (in Figure 4.3 they are shown by the dashed line in region I).

This explanation is supported by some experiments in which a deviation from the smooth dependence shown for the $\mathrm{SF}_{6}$ molecule in Figure 4.2 was observed. The $\bar{n}(\Phi)$ dependence for $\mathrm{SF}_{6}$ at low temperature, for example, is faster than the linear one. ${ }^{211}$ This is accounted for by a reduced number of initial quantum states of the molecule under cooling and hence a decreased number of potential pathways of successive vibrational-rotational transitions of the molecule. In this case two- and three-photon pro- 
cesses of excitation directly to the vibrational state $v=2,3$ begin to be involved, their probability nonlinearly dependent of intensity. Another example: the accurately measured $\bar{n}(\Phi)$ dependence for a molecule with comparatively few atoms, such as $\mathrm{OsO}_{4}$ is stepwise by character with several ranges of saturation. ${ }^{210,212}$ This dependence is illustrated in Figure 4.2. It is explained by the fact that different groups of molecules in different initial states penetrate region II at quite different field intensities. This gives rise to steps in the dependence.

At the same time, the $\bar{n}(\Phi)$ dependence for simple molecules (OCS, $\mathrm{D}_{2} \mathrm{O}$, etc.) measured in Ref. 203 is similar by character to the saturated absorption of a two-level system with a maximum number of absorbed photons $\bar{n}<1$ (Figure 4.2). This means that for such molecules the value of $P(\mathrm{I})$ critical for MP absorption at lower vibrational transitions is too high and cannot be attained in experiments. In essence, the $\bar{n}(\Phi)$ dependence measured for a molecule (see Section 5 of the next paper III) gives a good and reliable idea of the response of the molecule to MP dissociation without the need for direct observation of dissociation.

\subsubsection{Resonant character of MP absorption}

The dependence of the number of absorbed photons $\bar{n}$ on the $\Omega$ frequency called the MP absorption spectrum has a well-pronounced resonant behavior. It is just this property that enables selective MP excitation of molecules and particularly its application for laser isotope separation. Therefore, the resonant characteristics of MP absorption are important in practice and their research receives primary attention. By now measurements have been taken of the MP absorption spectra for dozens of molecules, mainly in the region of 9 to $11 \mu \mathrm{m}$ of a $\mathrm{CO}_{2}$ laser. The basic features of MP absorption spectra are: high sensitivity to radiation intensity, broadening and "red" resonance shift with increasing intensity, and formation of distinct narrow resonances in the spectrum in a certain intensity range.

Figure 4.4 shows the evolution of the MP dissociation spectrum for the $\mathrm{SF}_{6}$ and $\mathrm{OsO}_{4}$ molecules as the intensity increases. In the case of $\mathrm{SF}_{6}$ one can observe a well-pronounced shift of the absorption maximum to the long-wave length side and a broadening of the absorption band. This result was obtained in Ref. 203 by the opticoacoustic measurement of MP absorption and later was confirmed in Ref. 213 by measuring the transmission 

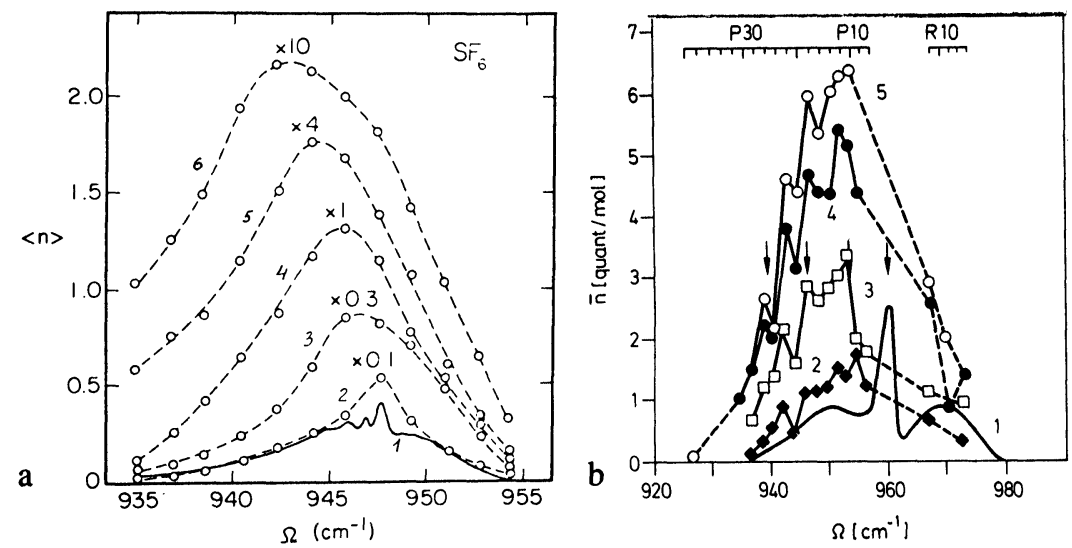

FIGURE 4.4 Evolution of the MP absorption spectra of $\mathrm{SF}_{6}$ and $\mathrm{OsO}_{4}$ molecules $(300 \mathrm{~K}$ ) with increasing IR pulse energy or intensity. Solid curves I show the linear absorption spectrum: a) curves $2,3,4,5$ and 6 are attained at radiation intensities of $0.035,0.15,1.2$, 10 and $60 \mathrm{MW} / \mathrm{cm}^{2}$ with $100 \mathrm{~ns}$. The scale of the curves on the ordinate must be increased by the factor given for each curve (from Ref. 203); b) curves 2, 3, 4 and 5 correspond to intensities of 3.5, 7.1, 10.5 and $14 \mathrm{MW} / \mathrm{cm}^{2}$ (from Ref. 210).

of a gas cell. The shift and broadening of the MP absorption band with increasing intensity can be qualitatively accounted for by vibration anharmonicity. As the IR radiation intensity increases, the average level of molecular excitation and hence the contribution of higher vibrational transitions to MP absorption increase, too. Since the spectrum of transitions between high vibrational levels due to anharmonicity shifts to the longwave length side with an increase in the number, this brings about a gradual "red" shift and broadening of the MP absorption band.

It must be noted that at room temperature only $30 \%$ of the molecules are in the ground vibrational state while the rest are under some equilibrium distribution over the other low-lying vibrational levels. This gives rise to "hot" bands in the linear absorption spectrum (the long-wave length peaks on the solid curve in Figure 4.4a). The distribution of molecules over many initial states greatly complicates, of course, the MP absorption spectrum since many different pathways of MP excitation of the molecule become possible. When $\mathrm{SF}_{6}$ is cooled down, this leads to a narrowing of the linear IR absorption spectrum ${ }^{214}$ because of the decreased intensity of "hot" bands and the decreased width of molecular distribution over rotational levels. 
This changes at once the MP absorption spectrum in which some structure appears. ${ }^{211,215}$

In some cases the structure in the MP absorption spectrum appears in a definite intensity range: at room temperature, for example, in the case of the $\mathrm{OsO}_{4}$ molecule (Figure 4.4b). ${ }^{210}$ With the IR pulse intensity $P>30$ $\mathrm{kW} / \mathrm{cm}^{2}$ for $\mathrm{OsO}_{4}$ the absorption varies from the linear one (Figure 4.3) and three peaks appear in the MP absorption spectrum at the $\mathrm{CO}_{2}$ laser lines. Their position agrees with the successive anharmonic shifts $\left(2\left|X_{33}\right|=5.98 \mathrm{~cm}^{-1}\right)$ of the $\nu_{3}$ mode under excitation. With an increase of $P$ the position of the peaks does not change but their contrast is reduced and the MP absorption band width increases.

The MP absorption spectra given in Figure 4.4a,b have been taken at comparatively low resolution (about $2 \mathrm{~cm}^{-1}$ ) in experiments with discrete tuning of the $\mathrm{CO}_{2}$ laser oscillation frequency at different rotational-vibrational lines. It is of greater interest to produce MP absorption spectra with higher resolution. Such experiments were performed in Ref. 216 with the use of a high-pressure $\mathrm{CO}_{2}$ laser with its continuous tuning of generation frequency. In experiments with $\mathrm{C}_{2} \mathrm{H}_{4}$ molecules the formation and evolution of the narrow resonances of MP absorption were studied. With the laser radiation energy fluence ranging from $10^{-3}$ to $10^{-2} \mathrm{~J} / \mathrm{cm}^{2}$ (or the intensity varying between $10^{4}$ and $10^{5} \mathrm{~W} / \mathrm{cm}^{2}$ ), one could observe the formation of very narrow resonances of MP absorption just $10^{-2} \mathrm{~cm}^{-1}$ wide. The appearance of such resonances shows that the MP excitation of molecules at lower vibrational transitions does occur very effectively because of the multistep and multiphoton resonances in a multilevel system. Narrow resonances of $\mathrm{MP}$ absorption have also been obtained for the $\mathrm{SF}_{6}$ molecule with the use of a continuously tunable $\mathrm{CO}_{2}$ laser. ${ }^{217}$

In conclusion it should be emphasized that the resonant absorption of a large number of IR photons in these cases is a property of any isolated polyatomic molecule even in the absence of any collisions. This is an essential difference from the resonant absorption of IR radiation by a molecular gas when there are many collisions between the molecules during the irradiation time. In that case the absorption of a large number $\bar{n}$ of IR photons per molecule has a trivial explanation: vibrational energy exchange between molecules during collisions (see Figure 1.2b). Regardless, MP excitation of a polyatomic molecule can be observed distinctly under condition (4.4), i.e., in the absence of any collisions, for example, in experiments with molecular beams ${ }^{218,219}$ and molecular ions isolated in vacuum with the use of electric and magnetic fields. ${ }^{206}$ 


\subsubsection{Fraction of MP excited molecules}

In the previous section when discussing the collisionless absorption of a large number of IR photons by a polyatomic molecule we had to deal with the concept of a relative fraction of molecules participating in MP absorption. This concept is a basic one in interpreting MP excitation and dissociation of molecules and must be considered specially. At small IR pulse intensities when the MP excitation of vibrational levels is not yet dominant, the monochromatic IR field in the absence of rotational relaxation interacts only with a small fraction of molecules at the rotational $J$ levels for which the resonant transition $v=0 \rightarrow v=1$ at the field frequency $\Omega$ is possible. The thermal population of these rotational levels determines the maximum fraction of molecules $f$ that can be excited by a short pulse to the level $v=1$ as the vibrational-rotational transition is saturated. This effect called the "rotational bottle neck" was predicted in Ref. 192 and observed experimentally in Ref. 193. In the foregoing Section (Section 3.3.2) it is shown how this effect has influence on the excitation of the vibrational-rotational transition. The value of $f$ for different molecules lies in the range between 0.1 and 0.001 and is highly dependent on gas temperature.

In the first experiments on MP absorption ${ }^{209,220}$ attempts were made to allow for this effect for estimating the real number of photons absorbed by the $\mathrm{SF}_{6}$ molecules involved in the process of MP excitation. For this purpose the absorbed energy was distributed only among the $f$-th fraction of molecules in the region of irradiation. Such a rough estimate caused the number of IR photons absorbed by a molecule to be initially overstated greatly. In Ref. 221, by observing the bleaching of the IR absorption band simultaneously at many vibrational-rotational transitions it was concluded that many initial rotational states were depleted under the action of a powerful IR pulse. This experiment shows that the fraction of $\mathrm{SF}_{6}$ molecules $q(\Phi)$ involved in the process of MP absorption with $\Phi=10^{-2}$ to $1.0 \mathrm{~J} / \mathrm{cm}^{2}$ is much higher than the fraction of molecules $f$ interacting with the IR field in the linear limit, i.e.,

$$
q(\Phi) \gg f=q(0)
$$

The most important question, however, is to what extent the value of $q(\Omega)$ is close to unity at some intensity or energy fluence. The answer to this question was given in experiments with $\mathrm{OsO}_{4},{ }^{222} \mathrm{SF}_{6}{ }^{223}$ and $\mathrm{CF}_{3} \mathrm{I} .{ }^{224}$ 


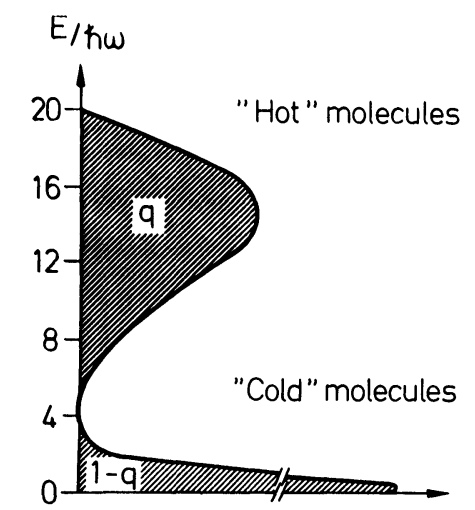

a)

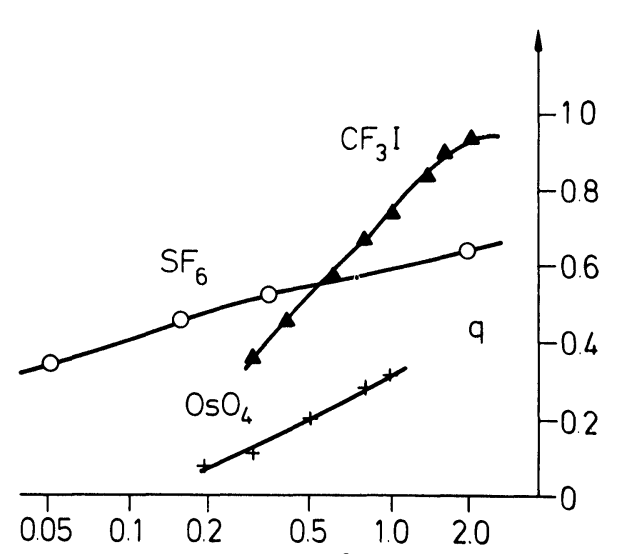

b)
$\Phi\left[\mathrm{J} / \mathrm{cm}^{2}\right]$

FIGURE 4.5 Formation of two ensembles of vibrational "hot" and "cold" molecules under MP excitation by powerful radiation pulses: a) distribution of vibrational energy of molecules at MP excitation; b) dependence of the relative fraction of highly excited molecules $q$ on the energy fluence of $\mathrm{CO}_{2}$ laser pulse for $\mathrm{SF}_{6}$ (from Ref. 223), $\mathrm{OsO}_{4}$ (from Ref. 222) and $\mathrm{CF}_{3} \mathrm{I}$ (from Ref. 224).

In these experiments different methods were applied to find that the value of $q(\Phi)$ at MP excitation grew with an increase of $\Phi$ from comparatively small magnitudes to its maximum $(q=1)$. Thus, it has been shown that under typical conditions MP excitation forms two ensembles of molecules. Some part of molecules $q$ involved in the process of MP excitation forms an ensemble of vibrationally "hot", i.e., highly excited, molecules. The rest, part $(1-q)$ of molecules, populates the lower vibrational levels and forms an ensemble of vibrationally "cold" molecules which probably do not differ materially from their initial state (Figure 4.5a).

In other words, it has been found that the function of the molecular vibrational energy distribution $F\left(E_{\text {vib }}\right)$ after MP excitation can be approximately presented as

$$
F\left(E_{\mathrm{vib}}\right)=q(\Phi) F_{\mathrm{hot}}\left(E_{\mathrm{vib}}\right)+(1-q) F_{\text {cold }}\left(E_{\mathrm{vib}}\right),
$$

where $F_{\text {cold }}\left(E_{\mathrm{vib}}\right)$ is the vibrational distribution describing the ensemble of "cold" molecules, and $F_{\text {hot }}\left(E_{\mathrm{vib}}\right)$ is the vibrational distribution of highly excited molecules describing the ensemble of "hot" molecules. The average number of absorbed IR photons $\bar{n}$ introduced above for all the molecules 


$$
\bar{n} \hbar \Omega=\int_{0}^{\infty} E_{\mathrm{vib}}\left[F\left(E_{\mathrm{vib}}\right)-F_{0}\left(E_{\mathrm{vib}}\right)\right] d E_{\mathrm{vib}}
$$

is related to the average number of photons $\bar{n}_{q} \simeq\langle n\rangle$ absorbed by the molecules of the "hot" ensemble

$$
\bar{n}_{q} \hbar \Omega=\int_{0}^{\infty} E_{\mathrm{vib}}\left[F_{\mathrm{hot}}\left(E_{\mathrm{vib}}\right)-F_{\mathrm{cold}}\left(E_{\mathrm{vib}}\right)\right] d E_{\mathrm{vib}}
$$

by the simple relation

$$
\bar{n}(\Phi, \Omega)=\bar{n}_{q}(\Phi, \Omega) q(\Phi, \Omega)
$$

where it is assumed that the average energy of the molecules in the hot ensemble $\bar{n}_{q} \hbar \Omega$ is much higher than the average energy of the molecules in the cold ensemble and $F_{\text {cold }}\left(E_{\mathrm{vib}}\right) \simeq F_{0}\left(E_{\mathrm{vib}}\right)$.

Figure 4.5b shows the dependences of the fraction of molecules $q$ excited to the vibrational quasicontinuum on the energy fluence $\Phi$ for three molecules obtained in Refs. 222-224. It can be clearly seen that with the IR pulse energy fluence ranging from 0.1 to $1.0 \mathrm{~J} / \mathrm{cm}^{2}$, typical of most experiments, only a fraction of molecules falls within the vibrational quasicontinuum. Therefore, it is impossible to measure correctly the average energy of the excited molecules $\bar{n}_{q} \hbar \Omega=E_{q}$ without taking into account the effect of "rotational" bottle neck or the effect of formation of two ensembles of molecules in our case. But as the energy fluence in the case of the above-mentioned molecules increases to several $\mathrm{J} / \mathrm{cm}^{2}$, it can easily be seen that all the molecules are involved to MP excitation, i.e., the only "hot" ensemble is formed. Under these conditions, when $q \simeq 1$, there is no need to take into account the effect of formation of two ensembles. To overcome the indeterminacy in estimating the fraction of molecules $q$ involved in MP excitation and to measure correctly the absorbed energy Bloembergen et al. ${ }^{208}$ used a subnanosecond pulse of $\mathrm{CO}_{2}$ laser for MP excitation. Even at a moderate energy fluence $\Phi \simeq 1 \mathrm{~J} / \mathrm{cm}^{2}$ such a pulse has high intensity (over $10^{9} \mathrm{~W} / \mathrm{cm}^{2}$ ), and therefore MP excitation of all the molecules, that is, the realization of the condition $q \simeq 1$, could be expected. This allowed measuring the dependence of the dissociation yield 
of molecules on the energy from the IR field without measuring the value of $q$.

Now that we know that the average number of absorbed IR photons per one molecule in the volume under irradiation $\bar{n}$ observed in the experiment is, in fact, the product of the fraction of excited molecules $q$ by the average level of their excitation $\bar{n}_{q}$, it is of interest to follow the origin of the resonant dependence $\bar{n}(\Omega)$. Such measurements of $q(\Omega)$ and $\bar{n}_{q}(\Omega)$ have been carried out for two molecules: $\mathrm{OsO}_{4}{ }^{222}$ and $\mathrm{CF}_{3} \mathrm{I}^{225}$ Figure 4.6 shows the dependence $q(\Omega)$. obtained in Ref. 222 with a fixed value of IR pulse energy fluence $\Phi=1.2 \mathrm{~J} / \mathrm{cm}^{2}$. At the maximum of linear IR absorption band $q=0.6$ and drops drastically at the long-wave length edge of the band. Figure 4.6 also shows the frequency dependence of the average energy $\bar{n}_{q}$ of the molecules in the "hot" ensemble. The number of IR photons absorbed by the "hot" molecules, on the contrary, increases at the long-wave length edge. This is due to an increase of the absorption crosssection in the vibrational quasicontinuum within the long-wave region.

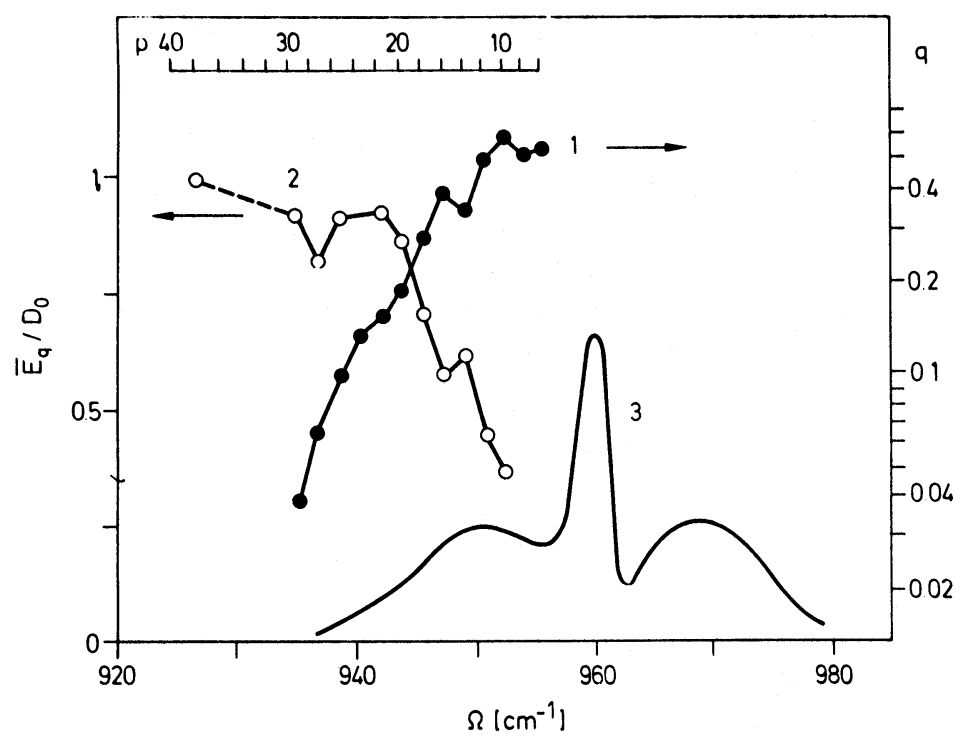

FIGURE 4.6 Dependence of the relative fraction $q$ of highly excited $\mathrm{OsO}_{4}$ molecules and their average energy $E_{q}=\bar{n}_{q} \hbar \Omega$ (in terms of dissociation energy) on the $\Omega$ frequency of IR radiation pulse with its energy fluence $\Phi=1.2 \mathrm{~J} / \mathrm{cm}^{2}$ and gas pressure of 0.03 Torr (from Ref. 222). 
Thus it can be concluded that the resonant character of MP absorption spectra, i.e., the $\bar{n}(\Omega)$ value determined, according to (4.12), from the product of $\bar{n}(\Omega)$ by $q(\Omega)$ is related to the resonant dependence of the fraction of molecules penetrating to the region of vibrational quasicontinuum.

\subsection{MP excitation of molecules in the vibrational quasicontinuum}

The MP excitation of polyatomic molecules in region II, that is the vibrational quasicontinuum, materially depends on the strong interaction of a large number of vibrational modes. Such interaction, first, gives rise to a vibrational quasicontinuum in which, instead of narrow resonances of MP absorption, a wide smooth absorption resonance appears around every vibrational mode which is active in IR absorption. Second, mixing of vibrational modes results in the stochastization of vibrational energy absorption among all vibrational modes. This specific feature of MP excitation of the vibrational quasicontinuum is qualitatively considered below.

\subsubsection{Evolution of MP absorption spectrum in the vibrational quasicontinuum}

The dependence of the MP absorption spectrum on the IR field frequency can be clearly seen from the experimental results for the $\mathrm{SF}_{6}$ and $\mathrm{OsO}_{4}$ molecules in Figure 4.4. The experimental dependences $\bar{n}(\Omega)$ observed, however, depend on the MP absorption spectrum both at lower vibrational transitions and in the vibrational quasicontinuum. It is very important to obtain separately the data on the MP absorption spectrum in regions I and II.

The first direct experimental data on the vibrational quasicontinuum of polyatomic molecules and the transition spectrum in it was obtained from the experiments ${ }^{226,227}$ on MP dissociation of molecules in a two-frequency IR field. A comparatively weak IR field with its frequency $\Omega_{1}$ resonantly excited the molecules from the ground vibrational state into the vibrational quasicontinuum, and the second rather intense IR field, with its frequency $\Omega_{2}$ detuned from the resonance frequency of unexcited molecules, performed MP excitation of molecules in the vibrational quasicontinuum up to the dissociation limit. In this method the functions of resonant excitation of molecules at lower transitions and excitation in the vibrational quasicontinuum between two pulses of different frequencies and intensities are separated. This technique allows the separate study of the transition spec- 


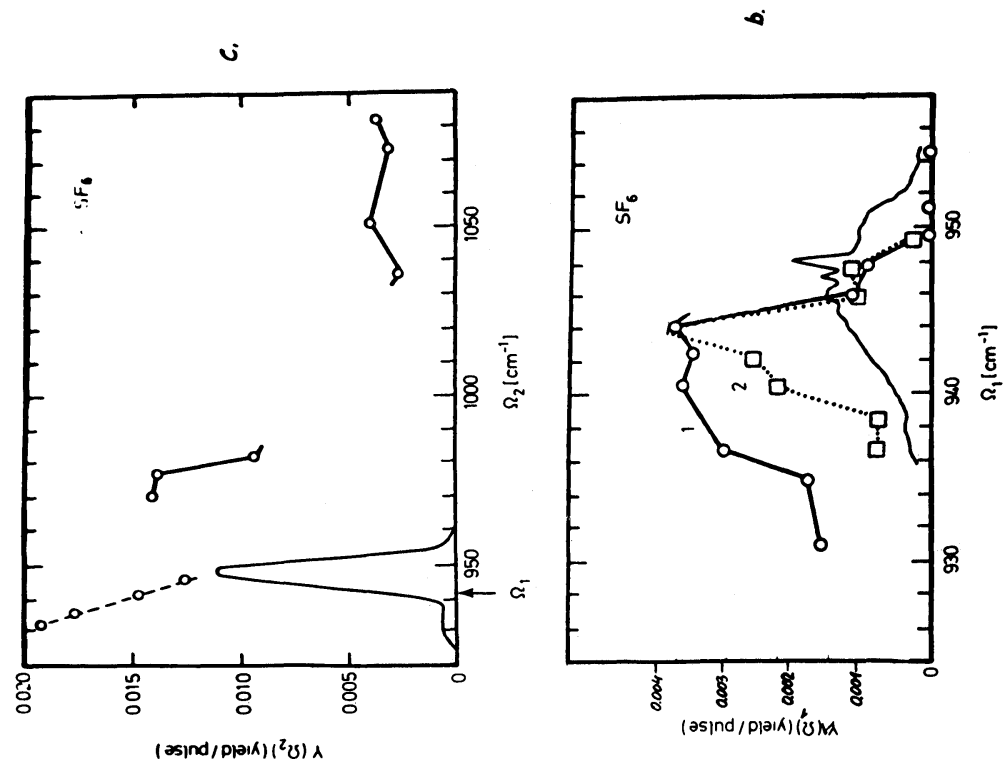

폼

颉

要

$4>0$

ํㅗㅀ

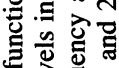

웛 유

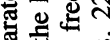

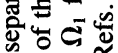

类

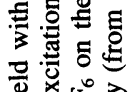

可㟧

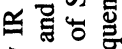

정정월

远

总造总

可焉

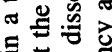

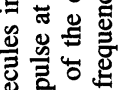

莙怘

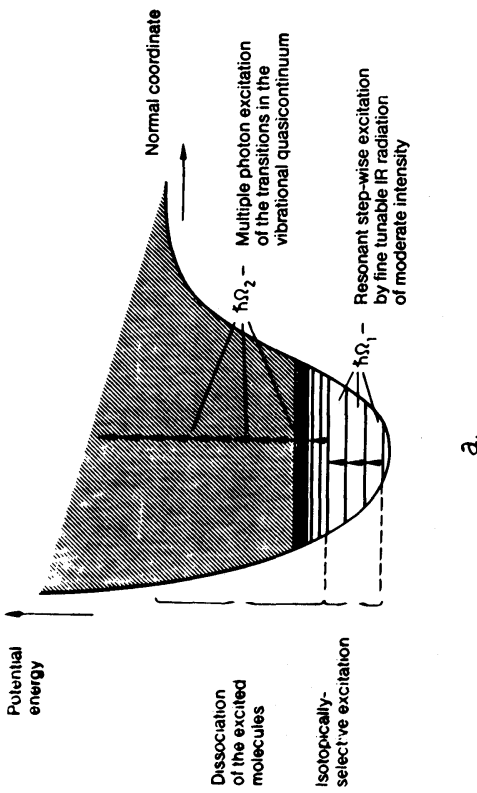

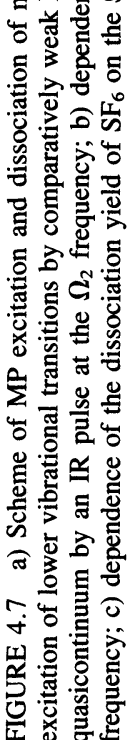


trum in the vibrational quasicontinuum. The frequency dependence of the dissociation yield $\beta$ can give information on the excitation efficiency of the vibrational quasicontinuum at different frequencies.

Figure 4.7a shows the general idea of MP excitation and dissociation of a polyatomic molecule in a two-frequency IR field and the results of the first experiment ${ }^{226,227}$ for the $\mathrm{SF}_{6}$ molecule. In Figure $4.7 \mathrm{~b}$ one can clearly see the resonance dependence of MP excitation on the $\Omega_{1}$ frequency of the first laser pulse interacting with the molecules at lower vibrational transitions. At the same time the dependence of the dissociation yield on the $\Omega_{2}$ frequency of the second pulse (Figure 4.7c) is less obvious. In this experiment the $\mathrm{CO}_{2}$ laser frequency $\Omega_{2}$ was tuned unfortunately only to the blue region about the absorption band of $\mathrm{SF}_{6}$. Therefore, it may possibly be the nonresonant MP excitation of $\mathrm{SF}_{6}$ molecules due to absorption at the short-wave length wing of the wide absorption band in the vibrational quasicontinuum that was observed. In subsequent experiments with $\mathrm{SiF}_{4},{ }^{228}$ $\mathrm{SF}_{6}{ }^{228,229}$ and $\mathrm{OsO}_{4}{ }^{230}$ one could observe a large shift of the maximum of the $\beta\left(\Omega_{2}\right)$ dependence to the long wave length spectral region.

It should be noted that, owing to the exponential dependence of the $\beta$ dissociation yield (see Section 2.4 in Ref. 202) on absorbed energy, a comparatively small increase of the absorption cross-section in the vibrational quasicontinuum brings about a large increase of $\beta$. Therefore, for direct measurement of the absorption spectrum in the vibrational quasicontinuum it is better to measure the frequency dependence of the energy fluence $\Phi($ II) which causes strong excitation of the transitions in the vibrational quasicontinuum and hence molecular dissociation. Such measurements were taken for $\mathrm{OsO}_{4}{ }^{230}$ and $\mathrm{SF}_{6},{ }^{228}$ and the value of $\Phi(\mathrm{II})$ was found to decrease from about $1 \mathrm{~J} / \mathrm{cm}^{2}$ to $0.1 \mathrm{~J} / \mathrm{cm}^{2}$ as $\Omega_{2}$ was red-shifted.

The experiments on MP dissociation of molecules in a two-frequency IR field as well as the experiment ${ }^{231}$ on measuring the IR absorption spectrum of heated $\mathrm{SF}_{6}$ gas (Figure 2.12 in Ref. 202) show that the spectrum of excited molecule in the vibrational quasicontinuum is concentrated near its frequencies active in IR absorption. The maxima of these bands shift to the long-wavelength side because of vibration anharmonicity as the $E_{\mathrm{vib}}$ vibrational energy increases.

In some works ${ }^{225,232}$ the models of absorption spectrum in the vibrational quasicontinuum are considered (see Section 2.3.4 in Ref. 202). It has been found that the experimental data can be described with the use of the Lorentzian contour for the spectral density of the square of the matrix element $\mu^{2}\left(E_{\mathrm{vib}}\right)$ or absorption cross-section $\sigma\left(\Omega, E_{\mathrm{vib}}\right)$ 


$$
\sigma_{\mathrm{qc}}\left(\Omega, E_{\mathrm{vib}}\right)=\sigma_{\mathrm{int}}\left(E_{\mathrm{vib}}\right) \frac{1}{\pi} \frac{\delta\left(E_{\mathrm{vib}}\right)}{\left[\Omega-v_{\max }\left(E_{\mathrm{vib}}\right)\right]^{2}+\delta^{2}\left(E_{\mathrm{vib}}\right)},
$$

where $\nu_{\max }\left(E_{\mathrm{vib}}\right)$ and $\delta\left(E_{\mathrm{vib}}\right)$ describe the center frequency and the halfwidth of the absorption band from a state with energy $E_{\mathrm{vib}}$ in the vibrational quasicontinuum. The simple model representations given in Section 2.3.4202 permit approximate estimations of the energy dependences $\sigma_{\text {int }}\left(E_{\mathrm{vib}}\right), \delta\left(E_{\mathrm{vib}}\right)$ and $\nu_{\max }\left(E_{\mathrm{vib}}\right)$.

First, the integral absorption cross-section $\sigma_{\text {int }}\left(E_{\mathrm{vib}}\right)$ gradually increases as the vibrational energy is increased. This is explained, of course, by an increase in the vibrational amplitude of a highly excited molecule. Figure 2.13 in Ref. 202 presents the calculated dependences $\mu^{2}\left(E_{\mathrm{vib}}\right)$ or $\sigma_{\text {int }}\left(E_{\mathrm{vib}}\right)$ on the vibrational energy $E_{\text {vib }}$ for the molecules $\mathrm{CF}_{3} \mathrm{I}$ and $\mathrm{SF}_{6}$. With $E_{\mathrm{vib}}$ increased by $20000 \mathrm{~cm}^{-1}$, the $\sigma_{\text {int }}$ cross-section increases by about 2 or 3 times.

Second, the absorption maximum frequency $\nu_{\max }\left(E_{\mathrm{vib}}\right)$ gradually shifts to the long-wavelength region with increasing vibrational energy. The value of such a shift, according to relation (2.82) from Ref. 202 depends linearly on the value of vibrational energy, and the coefficient of such a shift is determined by the anharmonicity constants.

Third, the absorption band width $\delta\left(E_{\mathrm{vib}}\right)$ grows with an increase of vibrational energy since the anharmonic interaction for highly excited states increases. The law of increase of $\delta\left(E_{\mathrm{vib}}\right)$ is determined by Fermi resonances, i.e., by the interaction of molecular vibrations. The dependence $\delta\left(E_{\mathrm{vib}}\right)$ should be treated specifically for every given molecule. For the $\mathrm{CF}_{3} \mathrm{I}$ molecule, for example, it is apparently the three-frequency Fermi resonances that play the basic part. Therefore, in Ref. 225 the dependence $\delta\left(E_{\mathrm{vib}}\right) \propto E_{\mathrm{vib}}^{3 / 2}$ is taken for this molecule.

To sum the data presented, Figure 4.8 shows in a simple way the twodimensional dependence $\sigma\left(\Omega, E_{\mathrm{vib}}\right)$ which gives a qualitative indication of the potential for MP excitation of a polyatomic molecule. The experimental dependence of the number of absorbed IR photons in a one-frequency field $\bar{n}(\Omega)$ results, roughly speaking, from successive superposition of such absorption spectra as the vibrational energy $E_{\text {vib }}$ increases. Therefore, the dependence $\bar{n}(\Omega)$ substantially shifts to the red region (Figure 4.4 ) with increasing radiation intensity which corresponds to an increase in $E_{\mathrm{vib}}$. In much the same way the decreasing of growth of the number of absorbed photons $\bar{n}$ with increasing energy fluence is related to the red shift of absorption band. As a result, the absorption cross-section for highly excited 


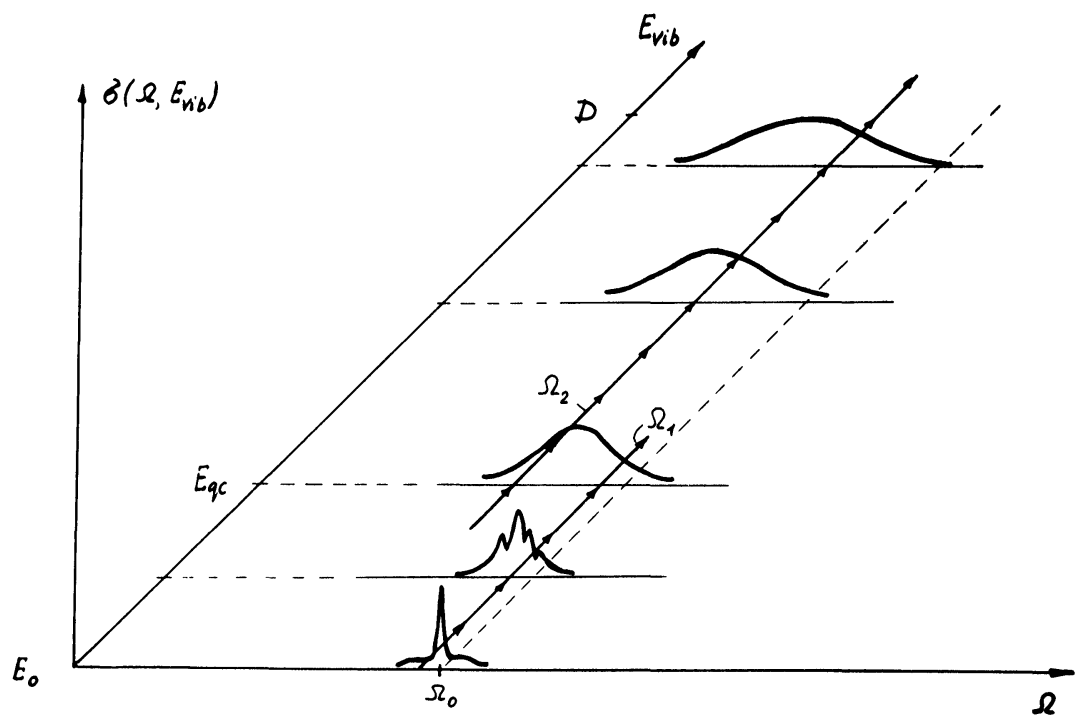

FIGURE 4.8 Qualitative evolution of the IR absorption spectrum of a polyatomic molecule in the vibrational quasicontinuum near the vibrational band as the vibrational energy of the molecule increases.

molecules at the fixed frequency $\Omega$ drops and their further excitation requires the higher energy fluence $\Phi$. So the experimental dependence $\bar{n}(\Phi)$ (see Figure 4.2) is usually somewhat weaker than the linear dependence that might be expected in the absence of an absorption band shift (Figure 4.3).

From the qualitative dependence $\sigma_{\mathbf{q c}}\left(\Omega, E_{\mathrm{vib}}\right)$ it can easily be seen that the efficiency of MP excitation and dissociation of a polyatomic molecule in a two-frequency IR field at properly chosen frequencies $\Omega_{1}$ and $\Omega_{2}$ is much higher. The excitation pathway of the molecule in this case is shown by arrows in Figure 4.8 . Thus, the IR absorption spectrum of an excited molecule $\sigma\left(\Omega, E_{\mathrm{vib}}\right)$ is a key characteristic for interpreting the MP excitation of a molecule. The quantitative description of molecule MP excitation calls for direct experimental data on the absorption spectrum of molecules in the vibrational quasicontinuum, at least the dependences of $v_{\text {max }}\left(E_{\text {vib }}\right)$ and $\delta\left(E_{\mathrm{vib}}\right)$. For this purpose, new methods of laser spectroscopy of highly excited vibrational states should be developed. In this case one can apparently apply the described method of excitation of molecules by two IR pulses with different frequencies $\Omega_{1}$ and $\Omega_{2}$. 


\subsubsection{Distribution of vibrational energy of a molecule under MP excitation}

A powerful IR radiation pulse usually acts on a molecule distributed over many initial states, i.e., those under different conditions. Under the action of an IR pulse the distribution of vibrational energy in the molecular ensemble becomes nonequilibrium. That is why two questions may arise: 1) what is the intermolecular distribution of vibrational energy in the ensemble of molecules under irradiation; 2) what is the intramolecular distribution of vibrational energy both over energy levels and over vibrational modes?

The intermolecular distribution of vibrational energy formed under a powerful IR pulse is essentially nonequilibrium due to the formation of two molecular ensembles: vibrationally excited molecules in the region of the vibrational quasicontinuum (a hot ensemble) and those left at the lower vibrational levels (a cold ensemble) as is shown in Figure 4.5.

In two cases, when the vibrational temperatures differ greatly, it is possible to avoid the formation of two such ensembles. First, when the molecules are excited by a very powerful short pulse with intensity $P \gg$ $P(\mathrm{I})$ it is possible to excite all the molecules to the vibrational quasicontinuum, i.e., to realize the condition $q \simeq 1$. $^{208}$ Second, a buffer gas inducing strong rotational relaxation of the excited molecules during a laser pulse but not causing their appreciable vibrational deactivation can be added into the molecular gas. This can always be done since the probability of change of rotational energy, as a polyatomic molecule comes into collision with an atom of the buffer gas, is much higher than the probability of change of vibrational energy. In this case collisions make it possible to suppress the effect of rotational bottle neck during MP excitation of molecules from many rotational states. Finally, after the laser pulse is over, the collisions of "vibrational-hot" and "vibrational-cold" molecules result in vibrational energy exchange between two ensembles and their combining into one ensemble with the same total vibrational energy.

The most direct information on intermolecular distribution of vibrational energy has been obtained in the experiments ${ }^{233}$ by the measurement of the Raman scattering spectrum in the vibrations of molecules excited by a $\mathrm{CO}_{2}$ laser pulse. Figure 4.9 presents the results of these experiments which vividly confirm the basic physical effects in MP excitation of molecules. At the top there is a Raman scattering spectrum in the Stokes region at the $v_{1}$ vibration of $\mathrm{SF}_{6}$ molecules without any excitation. When the molecules are excited by a $\mathrm{CO}_{2}$ laser pulse, an additional peak belonging to 


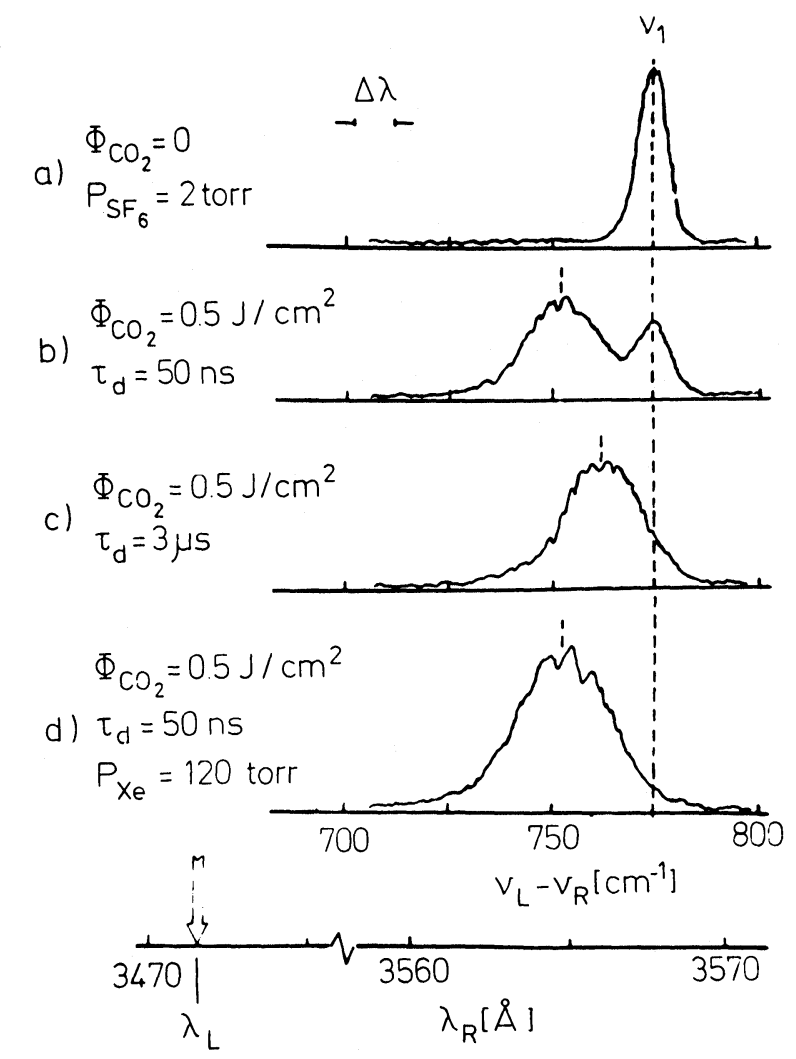

FIGURE 4.9 Spectra of Raman scattering Stokes signal of the second harmonic of ruby laser at the $\nu_{1}$ vibration of the $\mathrm{SF}_{6}$ molecule: a) unexcited $\mathrm{SF}_{6}$ molecules; $\mathrm{b}-\mathrm{d}$ ) the molecules are excited by a $\mathrm{CO}_{2}$ laser pulse with its energy fluence of $0.5 \mathrm{~J} / \mathrm{cm}^{2}$. The delay time of the ruby laser probe pulse about the $\mathrm{CO}_{2}$ laser exciting pulse $\tau_{d}=50 \mathrm{~ns}(\mathrm{~b}, \mathrm{~d})$ and $\tau_{d}=4 \mu \mathrm{s}$ (c). The last spectrum (d) is produced in the presence of buffer gas: Xe, 120 Torr (from Ref. 233).

the ensemble of hot molecules appears in the Raman scattering spectra (Figure 4.9b). This peak is a little smaller due to the anharmonic vibration shift and is shifted towards the Stokes region. This spectrum unambiguously points to the formation of two ensembles of vibration-excited molecules right after the $\mathrm{CO}_{2}$ laser pulse action is over. The ensembles combine into one because of vibrational exchange which is evident from the peak's disappearance when the delay between the $\mathrm{CO}_{2}$ laser pulse and the probing 
pulse for exciting the Raman scattering spectrum is increased to $3 \mu \mathrm{s}$ (Figure 4.9c). With a buffer gas added, rotational relaxation during MP excitation provides penetration of all the molecules from the lower levels into the vibrational quasicontinuum and only one ensemble of vibrationally hot molecules is formed (Figure 4.9d).

Intramolecular distribution of vibrational energy in the ensemble of hot molecules excited to region II is formed under the action of two processes: 1) stimulated upward and downward transitions under laser radiation; 2) mixing of vibrational energy between modes due to their interaction. In the presence of collisions, of course, particularly after the laser pulse is over, a Boltzmann distribution is expected to be attained in the hot molecular ensemble.

The shape of the vibrational distribution of molecules in the hot ensemble has not been so far measured in experiments. On the basis of the dissociation yield one can state indirectly that it really differs from the Boltzmann one. This agrees well with the calculation that, in the region of vibrational quasicontinuum, is rather reliable. The vibrational energy distribution in the region of the vibrational quasicontinuum can be calculated on the basis of the kinetic equations presented in the previous Section (Section 3.5).

An essential feature of the MP excitation of a polyatomic molecule in region II is the increasing density of vibrational states as $E_{\text {vib }}$ grows. According to the simple estimate given in Section 3.5.2., the ratio of state densities, with the vibrational energy changing by the $\hbar \Omega$ value which corresponds to absorption of one IR photon, increases by $\left(1+\hbar \Omega / E_{\mathrm{vib}}\right)^{s-1}$ times, where $s$ is the number of vibrational degrees of freedom. Therefore, the stimulated upward transitions for a polyatomic molecule $\left(E_{\mathrm{vib}} \rightarrow E_{\mathrm{vib}}\right.$ $+\hbar \Omega)$ prevail considerably over the downward transitions $\left(E_{\mathrm{vib}}+\hbar \Omega\right.$ $\left.\rightarrow E_{\mathrm{vib}}\right)$. Both the analytical ${ }^{232,234}$ and numerical ${ }^{196,218,219,225,235,236}$ computations show that for the real values of probability ratio of upward and downward transitions the distribution form $F\left(E_{\mathrm{vib}}\right)$ is rather close to the Poisson distribution (3.118). The difference in level density is only by factors of 1.5 to 2 times as the molecular energy changes by the energy of one $\mathrm{CO}_{2}$ laser quantum $\hbar \Omega$ turns out to be rather essential.

Vibrational distribution calculated in Ref. 225 using equation (3.123) and Boltzmann distribution at equal values of absorbed energy and at two values of laser pulse energy fluence. It may be seen that the calculated distribution of vibrational energy is somewhat "narrower" than the Boltzmann one and has an exponential "tail" that falls more rapidly. It can be easily understood from the note at the end of Section 3.5.3. Indeed, it 
should be noted that the absorption cross-section in the vibrational quasicontinuum $\sigma\left(\Omega, E_{\mathrm{vib}}\right)$ for $\mathrm{CF}_{3} \mathrm{I}$ has a red anharmonic shift $\nu_{\max }\left(E_{\mathrm{vib}}\right)$. Therefore, when the IR radiation frequency $\Omega$ is tuned to the center of the band at the lower transitions, the value of the cross-section $\sigma\left(\Omega, E_{\mathrm{vib}}\right)$ for highly excited transitions at the same frequency $\Omega$ gradually falls (see Figure 4.8). This leads to a "compression" of the vibrational distribution.

The difference of this vibrational distribution tail from the tail of the Boltzmann distribution at the same average number of absorbed photons $\langle n\rangle$ is quite observable in the experiment. The point is that it is the tail of the vibrational distribution lying above the dissociation limit $\mathrm{D}$ that contributes to the dissociation yield of the molecule (see Section 4.4). So the difference of the real distribution from the Boltzmann one manifests itself well in the dependence of the dissociation yield $\beta$ on absorbed energy.

After the laser pulse action is over, the Boltzmann distribution of populations in the hot ensemble of molecules must arise due to collisions. This gives a gradual Boltzmann tail in the distribution and can make a real contribution to the molecular dissociation after the laser pulse.

Vibrational energy stochastization of highly excited molecules in the region of the vibrational quasicontinuum has already been discussed in Section 2 (Section 2.3 ) of the foregoing paper. ${ }^{202}$ This property of highly excited molecules follows from very general principles of molecular dynamics. The vibrational motion is stochastic when the vibrational excitation energy $E_{\text {vib }}$ reaches the dissociation energy $D$. This conclusion indirectly follows from numerous measurements of the dependence of the molecular dissociation yield on the energy absorbed by a molecule. The results of such measurements (see Section 4.4 and the more comprehensive Section 7 in paper IV of the present series) are in good agreement with the statistical theory of monomolecular decay that is based materially on the assumption of vibrational excitation stochasticity.

The most important point, however, which is still not clearly understood is the position of the stochasticity energy limit $E_{\mathrm{qc}}$. This problem is of importance for molecular spectroscopy, multiphoton laser chemistry and the theory of nonlinear vibrations of systems with many degrees of freedom.

Section 2 (2.7) of the previous article ${ }^{202}$ is concerned with potential spectroscopic methods of measurement of the vibrational energy distribution. It is shown that the integral absorption factor in a vibrational band that is active in the IR spectrum must be weakly sensitive to the type of vibrational distribution and to the energy in this vibrational mode. Such a weak dependence is related only to the anharmonic terms responsible for 
the difference in the probability of upward and downward vibrational transitions. From this standpoint one should approach carefully the interpretation of a number of experiments ${ }^{237-239}$ based on the method of double IR-IR resonance. The authors believe that they observed the effect of vibrational energy stochastization in a molecule. In these experiments the $\mathrm{SF}_{6}$ gas was excited by a powerful laser IR pulse and the IR absorption of the probe laser beam was measured with a time resolution.

There are two processes for the reliable study of the vibrational motion stochastization in molecules where the molecular upward and downward transitions should be discriminated: spontaneous IR emission and antiStokes Raman scattering (RS). As is shown in Section 2 (Section 2.7) of the previous paper, ${ }^{202}$ the integral intensity of the spontaneous emission radiation band in the mode active in the IR spectrum and the integral intensity of the anti-Stokes Raman scattering band in the mode active in $\mathrm{RS}$ are proportional to the vibrational energy in the mode.

It is the method of spontaneous RS that has been applied for the direct observation of the vibrational energy stochastization in a highly excited molecule and to estimate the stochastization limit of vibrational energy. In this experiment, with the average energy of the $\mathrm{SF}_{6}$ and $\mathrm{CF}_{3} \mathrm{I}$ molecules in the hot ensemble varying in the vicinity of $10000 \mathrm{~cm}^{-1}$, the anti-Stokes Raman scattering from the second harmonic of ruby laser has been observed at all vibrational modes active in the Raman scattering spectrum. This unambiguously points to the excitation of these modes, and the excitation level remained constant as the probe pulse delay about the exciting pulse was varied in a range from $20 \mathrm{~ns}$ to $10^{-6} \mathrm{~s}$. At the same time, with a long delay $\tau_{\mathbf{d}}$ the vibrational energy distribution over all the modes must definitely have reached equilibrium from collisional vibrational exchange. Thus, the vibrational motion stochastization under the conditions of experiment ${ }^{240}$ occurs for a time shorter than 20 ns, i.e., during the very process of MP excitation. On the basis of such measurements ${ }^{241}$ it has been concluded that the energy limit of vibrational motion stochastization in the $\mathrm{SF}_{6}$ molecule is $3300 \mathrm{~cm}^{-3}$ higher than the initial vibrational energy at $300 \mathrm{~K}, E_{0}=700 \mathrm{~cm}^{-1}$, i.e., in the region of $v_{3} \simeq 4$. For a simpler molecule, like $\mathrm{CF}_{3} \mathrm{I}$, such measurements give $E_{\mathrm{qc}} \simeq 6000 \mathrm{~cm}^{-1}$. The methods of anti-Stokes RS seem to be used in systematic measurements of $E_{\mathrm{qc}}$ for many polyatomic molecules.

The excitation of molecules to high vibrational states lying in the region of the vibrational quasicontinuum by IR radiation is closely related to the problem of the response of nonlinear systems with many degrees of freedom 
to an external periodical force. This problem has been the goal of studies for a long time (see Ref. 242). The possibility of systematic studies into polyatomic molecules is of fundamental interest and these smallest nonlinear vibrational systems with many degrees of freedom, different in their structure and accessible in unlimited amounts for experimentalists, particularly in studying the energy limit and the time of full stochastization of vibrational energy, are very important.

The time of vibrational energy stochastization $\tau_{\text {stoch }}$ depends on anharmonic interaction and lies probably in the picosecond range. This range of IR pulse duration is very difficult to attain as yet for experimental measurements of $\tau_{\text {stoch }}$. Nevertheless, it has several promising possibilities. Specifically, of great interest is the situation when not all the vibrational degrees of freedom but only a certain part of them participates in the formation of transitions in the vibrational quasicontinuum near the frequency of the mode being excited. Such a situation, for example, may take place in complex molecules having spatially separated different functional groups. The anharmonic interaction between the vibrational modes of these functional groups may turn out to be rather weak in this case. The situation can exist when there is not an appropriate Fermi resonance for some molecular mode thac provides its coupling with the rest of the modes. In both cases it is probably possible to observe incomplete stochastization of vibrational motion in a molecule even for a time of about $10^{-9} \mathrm{~s}$. The probability of such incomplete stochastization, of course, increases considerably in the subnanosecond range of pulse duration.

\subsection{Dissociation of MP excitation of molecules in continuum}

A highly excited molecule within region III, that is, the region of a real continuum, may be chemically transformed even without collisions with other reacting partners due to various monomolecular reactions: dissociation, isomerization, dissociative ionization, etc. In this section we are going to restrict ourselves to considering the basic features of the most important and perfectly understood monomolecular process, that is, the dissociation of highly excited molecules.

\subsection{1. "Threshold" character of dissociation yield}

The dissociation yield, i.e., the probability of dissociation of a molecule acted upon by one IR radiation pulse has two very characteristic features: 
a sharp, almost threshold, dependence on the laser pulse energy fluence and a resonance dependence on the laser pulse frequency $\Omega$. The dependence $\beta(\Phi, \Omega)$ is restricted, of course, by the corresponding dependences of the MP absorption rate. These characteristics are considered in more detail below.

Even in the very first work, ${ }^{243,244}$ where the effect of visible luminescence of molecules in a strong IR field in the absence of collisions was revealed, the steep threshold character of the dependence of the luminescence intensity on the laser pulse intensity was observed. The threshold properties of the effect of MP dissociation were first studied comprehensively in Ref. 245 with the $\mathrm{SF}_{6}$ molecule. The dependence of the dissociation yield $\beta(\Phi)$ on the laser pulse energy fluence obtained in this work is given in Figure 4.10a. It may be seen that with $\Phi \simeq 2.0 \mathrm{~J} / \mathrm{cm}^{2}$ one can observe a distinct threshold of molecular dissociation. The decrease of the

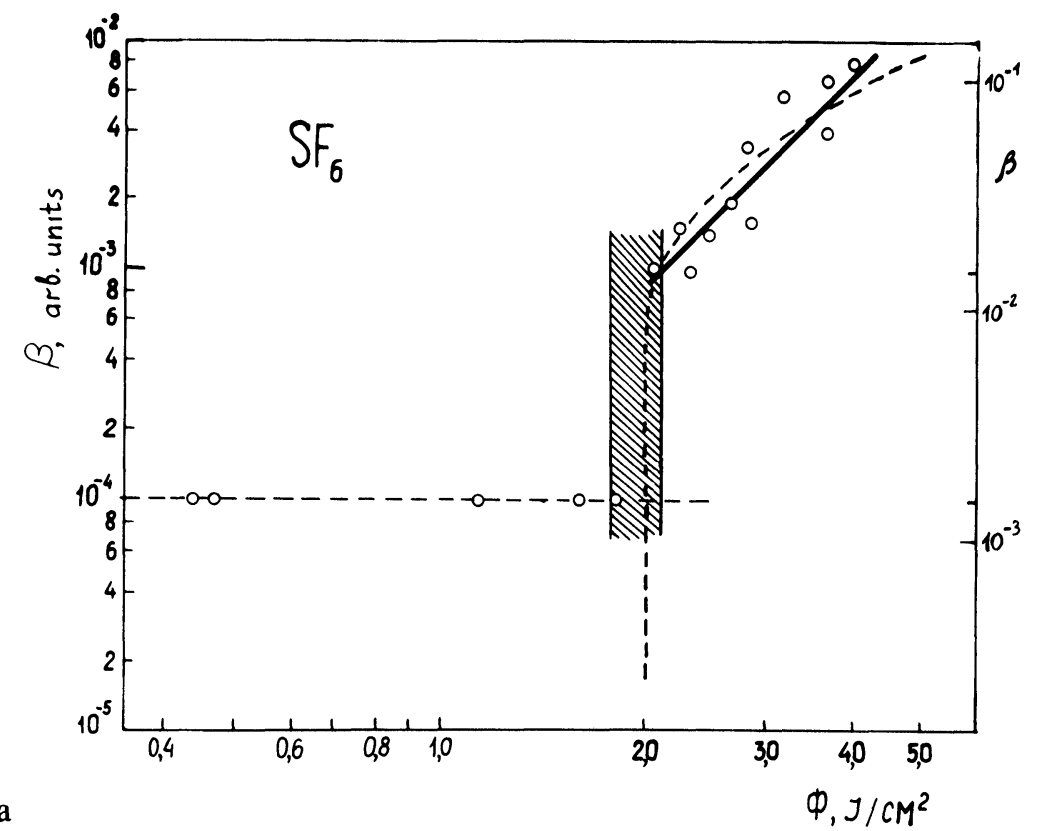

FIGURE 4.10 Dependences of the MP dissociation yield $\beta$ for the $\mathrm{SF}_{6}$ molecule (a) and the MP dissociation efficiency $\varphi$ for the $\mathrm{CF}_{3} \mathrm{I}$ molecules (b) on IR laser pulse energy fluence: a) $\beta$ in arbitrary units (from Ref. 247); b) the solid line stands for experimental and the dashed line for calculated (from Ref. 225). 
pulse energy by only $8 \%$ did not bring about any appreciable dissociation even though, with the energy fluence being below the threshold, the number of pulses essentially increased. With the threshold exceeded, the dependence of the dissociation yield was $\beta \propto \Phi^{3}$. In Ref. 246 the dissociation yield was measured as the $\mathrm{SF}_{6}$ molecule was acted upon by $\mathrm{CO}_{2}$ laser pulses of $0.5 \mathrm{~ns}$ and $100 \mathrm{~ns}$ duration. It was shown that the dissociation threshold was determined by the energy fluence rather than the intensity of the laser pulse. It must be said that the work changed the terminology. Before, the MP dissociation threshold had been related to laser radiation intensity (see, for example, Refs. 243-245 and 247. Figure 4.10a from Ref. 245 is given with the more correct terms.

In the first works dealing with the theory of MP dissociation some attempts were made to explain the threshold dependence. In these works the threshold property of the effect was associated with the molecule passing the lower levels lying below the vibrational quasicontinuum limit due to power broadening ${ }^{207,248}$ or with multiphoton transitions ${ }^{249,250}$ which are believed to reflect the exponential function $\beta(\Phi)$. But under such explanations the same steep dependence on $\Phi$ should have been observed for the value of absorbed energy $\bar{n}(\Phi)$ as well which does not correspond at all to the experimental data (see Section 4.1).

The key to the correct explanation of the threshold property of the effect of MP dissociation was found in Ref. 247 where the dependences $\beta(\Phi)$ were compared for the $\mathrm{SF}_{6}$ molecule as the bands of the $\nu_{3}$ mode active in IR absorption and the weak composite vibration $\nu_{2}+v_{6}$ were excited. It was found that, even though the value of dissociation yield in the case of $v_{2}+v_{6}$ was much smaller than for $v_{3}$, the thresholds for the two cases were almost identical. This experimental fact allows the threshold property to be associated with the excitation of molecules to the vibrational quasicontinuum which does not depend on how easily a molecule passes through the energy range below the vibrational quasicontinuum limit. In other words, under standard experimental conditions the energy fluence $\Phi($ II) that ensures effective excitation of molecules from region II to region III can be realized at intensities $P \gg P(\mathrm{I})$ which are quite sufficient for a significant fraction of molecules to pass from region I to region II (see Section 4.1). Excited molecules have vibrational energy distribution $F\left(E_{\mathrm{vib}}\right)$ with a sharp exponential fall-off towards high energies and an average number of $\left\langle E_{\text {vib }}\right\rangle$ proportional to the energy fluence $\Phi$. At increasing energy fluence $\Phi$ a small fraction of molecules in the vibrational distribution tail falls at first within the region of the real continuum. Then it exponen- 
tially grows as $\Phi$ increases. When the dissociation yield is measured in a limited region and with limited sensitivity, such an exponential dependence of the dissociation yield looks like a threshold dependence. More precise measurements give, of course, just the exponential dependence of the dissociation yield $\beta(\Phi)$ or $\beta(\langle n\rangle)$ (Figure 4.10a). This explanation of MP dissociation yield is now considered correct for molecules whose vibrational quasicontinuum limit lies not so high and which, therefore, dissociate at quite accessible laser pulse energy fluence $\left(1\right.$ to $\left.100 \mathrm{~J} / \mathrm{cm}^{2}\right)$.

If the laser energy fluence exceeds the threshold, the dissociation yield increases rapidly. When the value of $\beta$ becomes considerable, for example, about $0.2-0.3$, the growth of $\beta(\Phi)$ slows down and then tends to saturate. Figure $4.10 \mathrm{~b}$ illustrates such a dependence for the $\mathrm{CF}_{3} \mathrm{I}$ molecule. It should

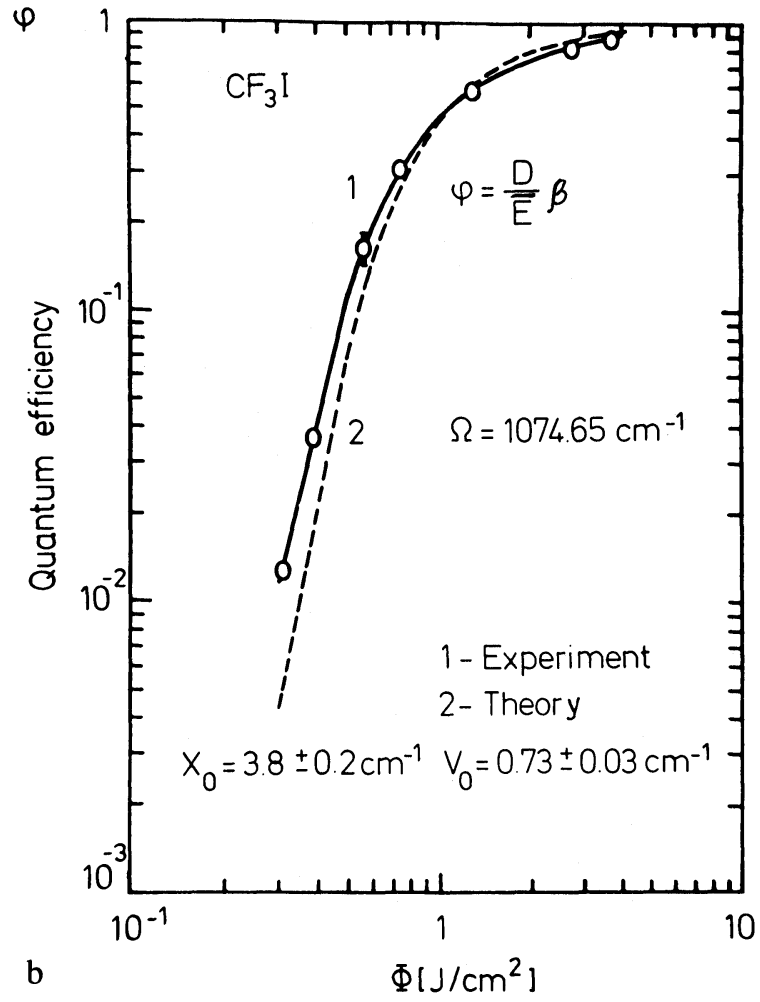

FIGURE 4.10 (continued) 
be noted that in this figure, instead of the dissociation yield per pulse $\beta$, on the ordinates the value of the so-called quantum efficiency of MP dissociation is plotted

$$
\varphi=\beta \frac{D}{\bar{n} \hbar \Omega}
$$

Physically it reflects more correctly the process of the dissociation of a highly excited molecule and is suited for comparison of theory with experiment. The point is that the dissociation yield per pulse is affected by two factors: the fraction of molecules $q$ falling within region II and the dissociation probability of a highly excited molecule depending on the absorbed energy $\bar{n}_{q}=\bar{n} / q$ (see Section 4.2.3). The value of $\varphi$ is apparently independent of $q$ since both a $q$ and $\bar{n}$ are proportional to the fraction of molecules $q$ falling within the vibrational quasicontinuum.

Since the absorption cross-section in the vibrational quasicontinuum, according to (4.13), depends on the IR radiation frequency, it follows from the approximated expression (4.3) that the energy fluence providing the excitation of molecules in region II to the limit of region III is frequencydependent, too. Therefore, to be precise, the "threshold" energy fluence providing a significant dissociation yield should also depend on the $\Omega$ frequency. The conclusion of Ref. 247 on the same dissociation threshold of $\mathrm{SF}_{6}$ for the bands $v_{3}$ and $\nu_{2}+v_{6}$ has been specified in Ref. 246 showing that in the case of the band $\nu_{2}+v_{6}$ the threshold is about twice as high than in the case of the band $v_{3}$. Moreover, even within one absorption band the change of the absorption cross-section in region II causes the threshold to change for different laser frequencies. ${ }^{451,452}$

The dependence of the MP dissociation yield $\beta$ on the MP absorption efficiency of IR radiation can be clearly seen in similar spectral dependences of dissociation yield and absorbed energy. Figure 4.11 shows the dependence of the dissociation yield of ${ }^{32} \mathrm{SF}_{6}$ on the pulse laser frequency $\Omega$ as it is excited within the $\nu_{3}$ band. ${ }^{245}$ The maximum of this dependence, as well as that of its corresponding dependence of absorbed energy (see Figure $4.4 a)$ is shifted to the long-wavelength spectral region. Even though the spectra of MP dissociation $\beta(\Omega)$ and MP absorption $\bar{n}(\Omega)$ are much wider than the IR absorption linear spectrum, in many cases the width of $\beta(\Omega)$ is considerably smaller than the isotope shift of absorption bands of different isotopic molecules. A typical example of such a situation is presented in Figure 4.11 for two isotopes $\left({ }^{32} \mathrm{~S}\right.$ and $\left.{ }^{34} \mathrm{~S}\right)$ of the $\mathrm{SF}_{6}$ molecule. ${ }^{245}$ From 


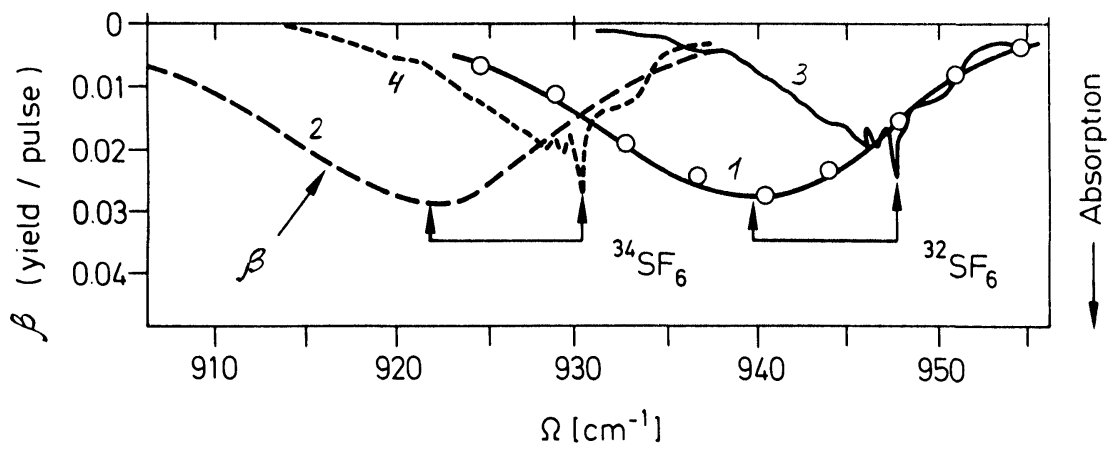

FIGURE 4.11 Dependence of the MP dissociation yield of $\mathrm{SF}_{6}$ on the IR radiation pulse frequency explaining the effect of isotopic selectivity of MP dissociation: 1) experimental dependence $\beta(\Omega)$ in arbitrary units for the ${ }^{32} \mathrm{SF}_{6}$ molecule, 2 ) assumed dependence $\beta(\Omega)$ for ${ }^{34} \mathrm{SF}_{6} ; 3$ ) and 4) spectra of linear absorption for ${ }^{32} \mathrm{SF}_{6}$ and ${ }^{34} \mathrm{SF}_{6}$ respectively (from Ref. 245 ).

the dependences presented it may be seen that as $\mathrm{SF}_{6}$ containing a mixture of such isotopic molecules as ${ }^{32} \mathrm{SF}_{6}$ and ${ }^{34} \mathrm{SF}_{6}$ is irradiated, there is no dissociation selectivity at the radiation frequency $\Omega_{0}$ at which the relative dissociation yields of both isotopic molecules are equal. As the frequency is shifted to the long-wavelength spectral range about $\Omega_{0}$, preferential dissociation of ${ }^{34} \mathrm{SF}_{6}$ must take place, and as it is shifted to the shortwavelength region, preferential dissociation for ${ }^{32} \mathrm{SF}_{6}$ molecules occurs. The $\beta(\Omega)$ dependences play a key role in choosing a radiation frequency as the effect of IR MP dissociation is applied as a method of laser isotope separation.

\subsubsection{Overexcitation of polyatomic molecules in continuum}

Under the action of a powerful IR pulse the nonequilibrium distribution of molecules over vibrational levels sets in (see Section 4.3.2). The distribution tail reaches the limit of a real continuum of states which corresponds to molecular dissociation. If the vibrational energy of a molecule exceeds the dissociation energy $D$ or in other words, the energy of breaking of the weakest bond $d_{0}$, the molecule is able to dissociate into fragments. The dissociation rate must evidently depend on the molecular state. For example, if the vibrational excitation energy is localized at the bond $d_{0}$, the dissociation must occur very quickly, during about one period of vibration. But if a considerable portion of energy is in other vibrational degrees of freedom, dissociation calls for fluctuation as a result of which 
the energy would be accumulated at the bond $d_{0}$. It takes a longer time than one period of vibration to realize this critical fluctuation. This is described by the expression (2.85) for monomolecular dissociation rate given in Section 2.4 of the previous paper. ${ }^{202}$

The simple formulas in Section 2.4 of Ref. 202 make it possible to estimate how strong the molecule can be excited over the dissociation limit due to its interaction with a laser IR pulse under typical experimental conditions when the pulse duration $\tau_{p} \simeq 100 \mathrm{~ns}$. If the energy fluence of a laser pulse exceeds the threshold value $\Phi$ (II) not very strongly, the number of absorbed IR photons is equal to the ratio $D / \hbar \Omega$. In this case the rate of passing of each successive transition connected with the absorption of one IR photon may be estimated as $k_{0} \simeq(D / \hbar \Omega) \tau_{p}^{-1}$. Since the typical values of $D / \hbar \Omega$ range from 20 to $50, k_{0} \simeq(2-5) 10^{8} \mathrm{~s}^{-1}$. Due to a rapid increase of the dissociation rate $k\left(E_{\mathrm{vib}}\right)$ the characteristic values of the $E_{\text {vib }}$ energy to which a molecule can be excited by an IR pulse can be estimated from the relation $k_{0} \simeq k\left(E_{\mathrm{vib}}\right)$ that corresponds approximately to $f\left(E_{\mathrm{vib}}\right) \simeq 10^{-4}$. Figure 2.14 in Ref. 202 shows the dependence $f\left(E_{\mathrm{vib}}\right)$ for different number $s$ of the vibrational degrees of freedom. It may be seen that for five-atom molecules the values $f\left(E_{\mathrm{vib}}\right) \simeq 10^{-4}$ can be obtained even with $\left(E_{\text {vib }}-D\right) \leqslant 0.10$, for six-atom molecules-with $\left(E_{\text {vib }}-D\right)$ $\leqslant 0.20$ and for seven-atom molecules-with $\left(E_{\text {vib }}-D\right) \leqslant 0.30$. Thus, for molecules with the number of atoms $N \leqslant 7$ the excitation over the dissociation limit under the action of a pulse with $\tau_{p} \simeq 10^{-7} \mathrm{~s}$ can actually be equal to $(0.1-0.3) D$. For more complex molecules and/or shorter pulses the value of overexcitation may even exceed the dissociation energy $D$. This is an important feature of IR photodissociation under transient pulsed conditions.

According to the statistical theory of dissociation, the dissociation products observed generally correspond to breakings of the weakest bond in a molecule. Direct diagnostics of the radicals resulting from MP dissociation of molecules became possible with the introduction of the molecular beam. ${ }^{218,253,254}$ This was used in Ref. 218 , for example, to prove that in the case of $\mathrm{SF}_{6}$ first an atom of $\mathrm{F}$ breaks away and then the dissociation of the $\mathrm{SF}_{5}$ radical gives rise to the $\mathrm{SF}_{4}$ radical. The alternative dissociation mechanism $\mathrm{SF}_{6} \rightarrow \mathrm{SF}_{4}+\mathrm{F}_{2}$ was rejected since there were no $\mathrm{F}_{2}$ molecules detected. Measuring the function of the kinetic energy distribution of the resulting radicals $g\left(E_{\mathrm{vib}}\right)$ enabled the authors of Ref. 218 to obtain information on the vibrational energy of the resulting radicals and hence on the degree of excitation of dissociating $\mathrm{SF}_{6}$ molecules over the dissociation 
limit. For example, with the energy fluence of a laser pulse $\Phi=4-10 \mathrm{~J} /$ $\mathrm{cm}^{2}$ the excess of $E_{\text {vib }}$ over $D$ comes to 6 to 10 photons of $\mathrm{CO}_{2}$ laser. This agrees well with the conclusions of statistical theory.

As discussed in Section 2.4 of Ref. 202, two different types of molecular dissociation are possible with their potential curves shown in Figure 4.12. In the first case the dissociation is not associated with rearrangement of chemical bonds, i.e., does not demand that the energy barrier should be overcome. This usually occurs when one atom breaks away, for example, an atom of $\mathrm{F}$ from $\mathrm{SF}_{6}$ molecule or an atom of $\mathrm{I}$ from the $\mathrm{CF}_{3} \mathrm{I}$ molecule. In this case the dissociation products have excess vibrational energy $\left(E_{\mathrm{vib}}\right.$ $-D$ ). In the other case, on the contrary, the chemical bonds get rearranged which requires that the energy barrier $E_{\mathrm{ac}}$ should be overcome. In this case a molecule must absorb an additional number of IR photons to overcome the energy barrier. The excess vibrational energy $\left(E_{\mathrm{ac}}-D\right)$ is transferred to the dissociation products. Such a case is characteristic of the breaking from a polyatomic molecule, for example a diatomic molecule, the atoms of which are not directly bound in the original molecule. A typical example for this case is the $\mathrm{CF}_{2} \mathrm{Cl}_{2}$ molecule that can dissociate not only through the channel $\mathrm{CF}_{2} \mathrm{Cl}_{2} \rightarrow \mathrm{CF}_{2} \mathrm{Cl}+\mathrm{Cl}$ (Figure 4.12a) but also $\mathrm{CF}_{2} \mathrm{Cl}_{2} \rightarrow \mathrm{CF}_{2}$ $+\mathrm{Cl}_{2}$ (Figure $4.12 \mathrm{~b}$ ). The dissociation probability of this molecule through the first channel is about one order of magnitude higher than through the
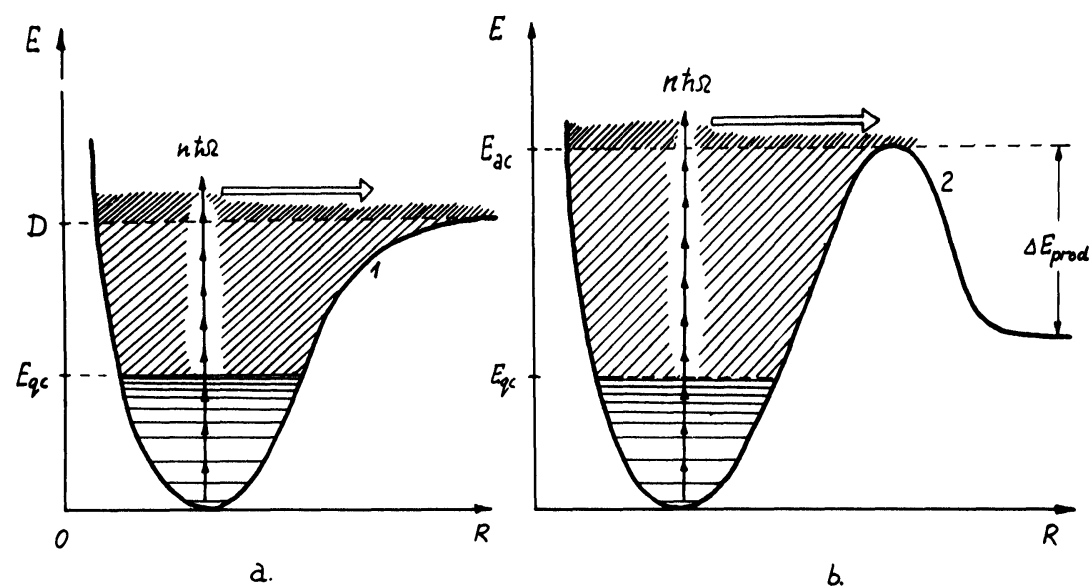

FIGURE 4.12 Schemes of potential curves leading to dissociation of a polyatomic molecule without a potential barrier (a) and in the presence of a potential barrier with its energy $E_{\text {ac }}$ and with transfer of excess energy $\Delta E_{\text {prod }}$ to the dissociation products (b). 
second one. ${ }^{255,256}$ This effect is caused by the presence of a potential barrier for the second reaction. This was proved by the measurement of the vibrational energy distribution for the $\mathrm{CF}_{2}$ radical in Ref. 257. It has been found that the vibrational temperature of this radical remains constant $\left(T_{v}\right.$ $=1050 \mathrm{~K}$ ) although the radical yield changes by an order of magnitude as $\Phi$ varies. This points to the fact that molecular dissociation takes place near the top of the barrier the height of which is $6900 \mathrm{~cm}^{-1}$.

Finally, we should emphasize an important peculiarity of MP excitation of polyatomic molecules with a large number of atoms, that is, their significant excitation over the dissociation limit $D$. In experiments this could be clearly observed ${ }^{258}$ in measuring the number of IR photons absorbed by one $\left(\mathrm{CF}_{3}\right)_{3} \mathrm{CI}$ molecule. The number of absorbed IR photons observed for this molecule varies between 35 and 40 which is about twice as much as the dissociation energy of the weakest bond C-I. At the same time, similar measurements for such a simpler molecule as $\mathrm{CF}_{3} \mathrm{I}$ result in $\bar{n} \simeq 20-22$, i.e., the number of IR photons absorbed in the real vibrational continuum $\left(E_{\mathrm{vib}}-D\right) / \hbar \Omega \leqslant 2 .{ }^{225}$

This particular feature of polyatomic molecules is essential during MP excitation by very short pulses when very high vibrational excitation of molecules over the dissociation limit can occur. In this case the degree of overexcitation $\left(E_{\text {vib }}-D\right)$ may be sufficient to overcome the next dissociation limits of a polyatomic molecule, and then the dissociation of the molecules through many channels becomes possible.

\subsection{MP excitation of the electronic states of polyatomic molecules by intense IR radiation}

Vibrationally highly excited molecules in the ground state can pass to excited electronic states. This can be due to nonadiabatic interaction of two electron terms (see Section 2.7 in the foregoing article ${ }^{202}$ ). The diagram of electron terms with possible $V \rightarrow E$ transition is illustrated in Figure 2.19. ${ }^{202}$ Some fraction of molecules can perform such transitions according to the difference in densities of vibrational states of molecules with their vibrational energy $E_{\text {vib }}$ in the ground electronic state $\rho_{0}\left(E_{\text {vib }}\right)$ and molecules with their vibrational energy $\left(E_{\mathrm{vib}}-E_{1}\right)$ in the excited electronic state $\rho_{1}\left(E_{\text {vib }}-E_{1}\right)$, where $E_{1}$ is the minimum energy limit of excited electron term. Between the molecules in two electronic states equilibrium sets in from direct $E \rightarrow V$ and reverse $V \rightarrow E$ transitions in accordance with the lifetimes of molecules with their vibrational energy $E_{\text {vib }}$ in the ground 
electronic state $\tau_{0}\left(0, E_{\mathrm{vib}}\right)$ and molecules with their vibrational energy $\left(E_{\mathrm{vib}}\right.$ - $\left.E_{1}\right)$ and electronic energy $E_{1}$ in the excited electronic state $\tau_{1}\left(E_{1}, E_{\text {vib }}\right.$ $\left.-E_{1}\right)$ :

$$
\frac{\rho_{0}\left(E_{\mathrm{vib}}\right)}{\tau_{0}\left(0, E_{\mathrm{vib}}\right)}=\frac{\rho_{1}\left(E_{\mathrm{vib}}-E_{1}\right)}{\tau_{1}\left(E_{1}, E_{\mathrm{vib}}-E_{1}\right)}
$$

If the excited electronic state is not metastable, usually $\tau_{1}\left(E_{1}, E_{\mathrm{vib}}-E_{1}\right)$ $\ll \tau_{0}\left(0, E_{\text {vib }}\right)$. Therefore, even with the vibrational level densities differing greatly $\left[\rho_{0}\left(E_{\mathrm{vib}}\right) \gg \rho_{1}\left(E_{\mathrm{vib}}-E_{1}\right)\right]$ a considerable fraction of vibrationally excited molecules can pass to an excited electronic state. In experiments this can be clearly seen as visible luminescence of molecular gas and appears even in the absence of dissociation. In essence, this process of conversion of vibrational energy to electronic energy ( $V \rightarrow E$ conversion) is typical of polyatomic molecules. Transitions with the electronic states of a molecule excited by IR radiation are often called "reverse nonradiative transitions". Such a process has been observed and studied for a number of polyatomic molecules, for example, $\mathrm{OsO}_{4}{ }^{259,260}$

In the excited electronic state a polyatomic molecule can perform induced upward transitions under the action of the same IR pulse. This is quite possible provided that the vibrational band of IR absorption remains in the excited electronic state. As a result, the molecule can be excited above the dissociation limit $D_{1}$ of the excited electronic term. In this case the dissociation of the molecule will give a molecular fragment in the excited electronic state (Figure 4.13a). Thus, the luminescence of molecular gas under powerful pulsed IR irradiation has, in principle, two origins: 1) luminescence of the original molecule in the excited electronic state; 2) luminescence of the dissociation products of the molecule through their excited electronic states. These processes can exist and compete even in one molecule, as has been observed, for example, in the cases of $\mathrm{OsO}_{4}{ }^{259}$ and $\mathrm{CrO}_{2} \mathrm{Cl}_{2}{ }^{260}$ molecules.

As noted in the foregoing Section 4.4, a polyatomic molecule with a large number of atoms may undergo strong excitation over the dissociation limit. In this case mixing of the vibrational states of a molecule in the real continuum for the ground electron term with the excited electron term may take place. Specifically, even the $V \rightarrow E$ transition of a highly excited molecule to the repulsive electron term of the molecule, that leads to an almost instantaneous dissociation of the molecule, is possible (Figure 4.13b). Due to a very short lifetime of the molecule $\tau_{1}$ at the repulsive electronic 


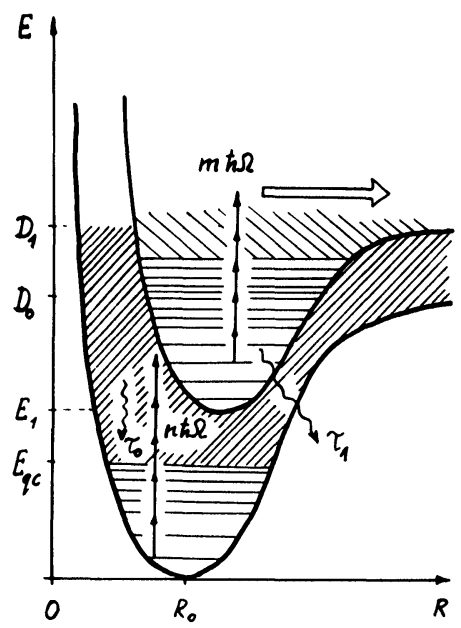

a.
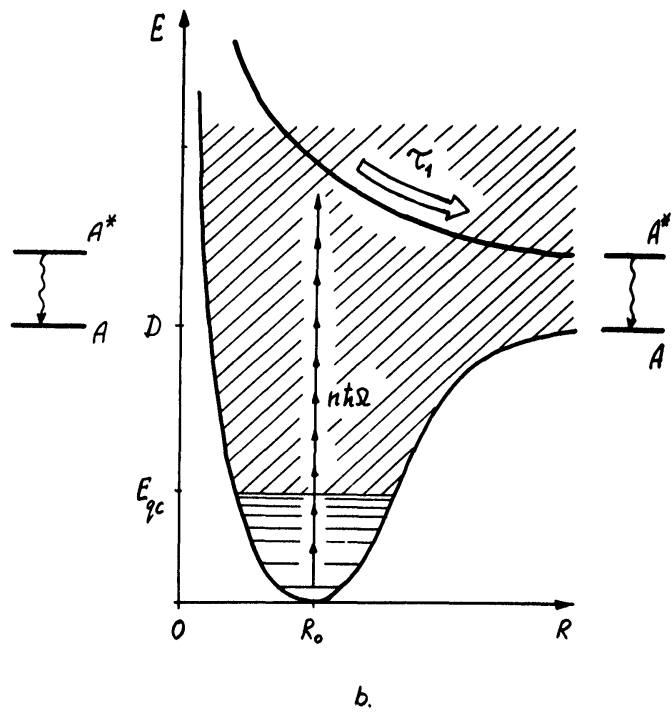

FIGURE 4.13 Different schemes of excitation of electronic states at MP vibrational excitation of a polyatomic molecule. The minimum energy of excited electronic state may lie below (a) and above (b) the dissociation limit.

term the process $E \rightarrow V$ almost does not come about, and, in fact, one can observe the electronic predissociation of the molecule due to the mixing of the nonparticipating electronic terms. Such a process was experimentally observed in Ref. 261 for the $\left(\mathrm{CF}_{3}\right)_{3} \mathrm{CI}$ molecule when electron-excited atoms were formed due to MP excitation of the original molecule.

\section{References}

174. R. Loudon. The Quantum Theory of Light. Clarendon Press, Oxford, 1973.

175. V. N. Bagratashvili, V. S. Letokhov, A. A. Makarov, E. A. Ryabov. Laser Chemistry (in press).

176. R. L. Shoemaker. In: Laser and Coherence Spectroscopy, ed. by J. I. Steinfeld, Plenum Press, New York-London, 1978, p. 197.

177. V. S. Letokhov, V. P. Chebotayev. Nonlinear Laser Spectroscopy, ed. by Springer Series in Optical Sciences, Vol. 4, Springer-Verlag, Berlin-New York, 1977.

178. P. G. Kryukov, V. S. Letokhov. Usp. Fiz. Nauk (Russian), 99, 169 (1969) [Sov. Phys. Uspekhi, 12, 641 (1970)]. 
179. I. A. Poluéktov, Yu. M. Popov, V. A. Roŭtberg. Usp. Fiz. Nauk (Russian), 114, 97 (1974) [Sov. Phys. Uspekhi, 17, 673 (1975)].

180. L. Allen, J. H. Eberly. Optical Resonance and Two-Level Atoms. John Wiley \& Sons, New York-London-Sydney-Toronto, 1975.

181. F. A. Novak, J. M. Friedman, R. M. Hochstrasser. In: Laser and Coherence Spectroscopy, ed. by J. I. Steinfeld, Plenum Press, New York-London, 1978, p. 451.

182. Ya. B. Zel'dovich. Usp. Fiz. Nauk (Russian), 110, 139 (1973) [Sov. Phys. Uspekhi, 16, $427(1973)]$.

183. R. P. Feynman, F. L. Vernon, R. W. Hellwarth. J. Appl. Phys., 28, 49 (1957).

184. V. S. Popov, A. M. Perelomov. Zh. Eksp. Teor. Fiz. (Russian), 56, 1375 (1969); 57, 1684 (1969) [Sov. Phys. JETP, 29, 738 (1969); 30, 910 (1969)].

185. C. D. Cantrell, V. S. Letokhov, A. A. Makarov. In: Coherent Nonlinear Optics, ed. by M. S. Feld and V. S. Letokhov, Topics in Current Physics, Vol. 21, SpringerVerlag, Berlin-Heidelberg-New York, 1980, p. 165.

186. P. F. Liao, T. E. Bjorkholm. Phys. Rev. Lett., 34, 1 (1975).

187. M. V. Kuz'min. Kvantovaya Elektronika (Russian), 5, 759 (1978) [Sov. J. Quant. Electr., 8, 438 (1978)].

188. L. D. Landau, E. M. Lifshitz. Mekhanika, Nauka, Moscow, 1965.

189. V. I. Gorchakov, V. N. Sazonov. Zh. Eksp. Teor. Fiz. (Russian), 70, 467 (1976) [Sov. Phys. JETP, 43, 241 (1976)].

190. M. V. Fedorov. Zh. Eksp. Teor. Fiz. (Russian), 73, 134 (1977) [Sov. Phys. JETP, 46, 69 (1977)].

191. F. Bloch. Phys. Rev., 70, 460 (1946).

192. V. S. Letokhov, A. A. Makarov. Zh. Eksp. Teor. Fiz. (Russian), 63, 2064 (1972) [Sov. Phys. JETP, 36, 1091 (1973)].

193. V. S. Letokhov, A. A. Makarov, E. A. Ryabov. Dokl. Akad. Nauk SSSR (Russian), 212, 75 (1973) [Sov. Phys. Dokl., 18, 603, (1974)].

194. A. A. Makarov, V. T. Platonenko, V. V. Tyakht. Zh. Eksp. Teor. Fiz. (Russian), 75, 2075 (1978) [Sov. Phys. JETP, 48, 1044 (1978)].

195. V. M. Akulin, A. M. Dykhne. Zh. Eksp. Teor. Fiz. (Russian), 73, 2098 (1977) [Sov. Phys. JETP, 46, 1099 (1977)].

196. M. Quack. J. Chem. Phys., 69, 1282 (1978).

197. S. Mukamel. In: Photoselective Chemistry, Part 1, ed. by J. Jortner, R. D. Levine, and S. A. Rice. Advances in Chemical Physics, Vol. XLVII, John Wiley \& Sons, New York-Chichester-Brisbane-Toronto, 1981, p. 509.

198. V. M. Akulin, A. M. Dykhne. Zh. Eksp. Teor. Fiz. (Russian), 81, 1262 (1981).

199. W. Feller. An Introduction to Probability Theory and its Applications, Vol. 1, John Wiley \& Sons, Inc. London Chapman \& Hall, Limited, 1958.

200. H. Bateman, A. Erdélyi. Higher Transcendental Functions, Vol. 2, McGraw-Hill Book Company, New York-Toronto-London, 1953.

201. V. S. Letokhov, A. A. Makarov. Optics Commun., 17, 250 (1976).

202. V. N. Bagratashvili, V. S. Letokhov, A. A. Makarov, E. A. Ryabov. Part I of this review paper Laser Chemistry, 1, (1982).

203. V. N. Bagratashvili, I. N. Knyazev, V. S. Letokhov, V. V. Lobko. Optics Comm., 18, 525 (1976).

204. D. Proch, H. Schroder. Chem. Phys. Lett., 61, 426 (1979).

205. T. B. Simpson, N. Bloembergen. Optics Comm., 37, 256 (1981).

206. D. S. Bomse, R. L. Woodin, J. L. Beachamp. In Advances in Laser Chemistry, ed. by A. N. Zewail, Springer Series in Chemical Physics, vol. 3 (Springer-Verlag, BerlinHeidelberg-New York) p. 94. 
207. N. Bloembergen. Optics Comm., 15, 416 (1975).

208. N. Bloembergen, E. Yablonovitch. In Laser Spectroscopy III, eds. J. L. Hall and J. L. Carlsten, Springer Series in Optical Sciences, Berlin-Heidelberg-New York, SpringerVerlag, vol. 7, p. 86 (1977).

209. R. V. Ambartzumian, Yu. A. Gorokhov, V. S. Letokhov, G. N. Makarov. Zh. Eksp. Teor. Fiz. (Russian) 69, 1956 (1975) [Sov. Phys. JETP, 42, 993 (1975)].

210. R. V. Ambartzumian, I. N. Knyazev, V. V. Lobko, G. N. Makarov, A. A. Puretzky. Appl. Phys., 19, 75 (1979).

211. S. S. Alimpiev, N. V. Karlov, B. G. Sartakov, E. M. Khokhlov. Optics Comm., 26, 45 (1978).

212. R. V. Ambartzumian, G. N. Makarov, A. A. Puretzky. Pis'ma Zh. Eksp. Teor. Fiz. (Russian), 28, 696 (1978) [JETP Lett., 28, 647 (1978)].

213. D. O. Ham, M. Rothshild. Optics Lett., 1, 28 (1977).

214. P. L. Houston, J. I. Steinfeld, J. Mol. Spectr., 54, 335 (1975).

215. T. F. Deutsch. Optics Lett., 1, 25 (1977).

216. I. N. Knyazev, N. P. Kuzmina, V. S. Letokhov, V. V. Lobko, A. A. Sarkisian. Appl. Phys., 22, 429 (1980).

217. S. S. Alimpiev, N. V. Karlov, G. A. Mesiatz, S. M. Nikiforov, V. M. Orlovsky, A. M. Prokhorov, B. G. Sartakov, E. M. Khokhlov, A. L. Shtarkov. Pis'ma Zh. Eksp. Teor. Fiz. (Russian), 30, 279 (1979) [Sov. Phys. JETP Lett., 30, 246 (1979)].

218. E. R. Grant, M. J. Coggiola, Y. T. Lee, P. A. Schulz, Aa. S. Sudvo, Y. R. Shen. Chem. Phys. Lett., 52, 595 (1977).

219. E. R. Grant, P. A. Schulz, Aa. S. Sudvo, M. J. Coggiola, Y. T. Lee, Y. R. Shen. In Laser Spectroscopy III, eds. J. L. Hall and J. L. Carlsten, Springer Series in Optical Sciences, Berlin-Heidelberg-New York, Springer-Verlag, vol. 7, p. 86 (1977).

220. R. V. Ambartzumian, N. V. Chekalin, Yu. A. Gorokhov, V. S. Letokhov, G. N. Makarov, E. A. Ryabov. In Laser Spectroscopy II, eds. S. Haroche et al., Lecture Notes in Physics, Berlin-Heidelberg-New York, Springer-Verlag, vol. 43, p. 121 (1975).

221. S. S. Alimpiev, V. N. Bagratashvili, N. V. Karlov, V. S. Letokhov, V. V. Lobko, A. A. Makarov, B. G. Sartakov, E. M. Khokhlov. Pis'ma Zh. Eksp. Teor. Fiz. (Russian) 25, 282 (1977) [Sov. Phys. JETP Lett., 25, 547 (1977)].

222. R. V. Ambarztumian, G. N. Makarov, A. A. Puretzky. Pis'ma Zh. Eksp. Teor. Fiz. (Russian) 28, 246 (1978) [Sov. Phys. JETP Lett., 28, 228 (1978)].

223. V. N. Bagratashvili, V. S. Doljikov, V. S. Letokhov. Zh. Eksp. Teor. Fiz. (Russian) 76, 18 (1979) [Sov. Phys. JETP 49, 8 (1979)].

224. V. N. Bagratashvili, V. S. Doljikov, V. S. Letokhov, E. A. Ryabov. In Laser Induced Processes in Molecules, ed. by K. L. Kompa, S. D. Smith, Springer Series in Chemical Physics, vol. 6, Berlin-Heidelberg-New York, Springer-Verlag,.p. 179 (1979).

225. V. N. Bagratashvili, V. S. Doljikov, V. S. Letokhov, A. A. Makarov, E. A. Ryabov, V. V. Tyakht. Zh. Eksp. Teor. Fiz. (Russian) 77, 2238 (1979) [Sov. Phys. JETP, 50, 1075 (1979)].

226. R. V. Ambartzumian, Yu. A. Gorokhov, V. S. Letokhov, G. N. Makarov, A. A. Puretzky, N. P. Furzikov. Pis'ma Zh. Eksp. Teor. Fiz. (Russian) 23, 217 (1976) [Sov. Phys. JETP Lett., 23, 194 (1976)].

227. R. V. Ambartzumian, N. P. Furzikov, Yu. A. Gorokhov, V. S. Letokhov, G. N. Makarov, A. A. Puretzky. Optics Comm., 18, 517 (1976).

228. V. M. Akulin, S. S. Alimpiev, N. V. Karlov, A. M. Prokhorov, V. G. Sarakov, E. M. Khokhlov. Pis'ma Zh. Eksp. Teor. Fiz. (Russian) 25, 428 (1977) [Sov. Phys. JETP Lett.].

229. M. C. Gower, T. K. Gustafson. Optics Comm., 23, 69 (1977).

230. R. V. Ambartzumian, V. S. Letokhov, G. N. Makarov, A. A. Puretzky. Optics Comm., 25, 69 (1978). 
231. A. V. Nowak, J. L. Lyman. J. Quant. Spectr. Rad. Transf., 15, 1945 (1975).

232. W. Fuss. Chem. Phys., 36, 135 (1979).

233. V. N. Bagratashvili, Yu. G. Vainer, V. S. Doljikov, V. S. Letokhov, A. A. Makarov, L. P. Maliavkin, E. A. Ryabov, A. G. Sil'kis. Optics Lett., 6, 148 (1981).

234. V. T. Platonenko. Kvantovaya Elektronika (Russian) 5, 1783 (1978) [Sov. J. Quant. Electr. 8, 1010 (1978)].

235. J. C. Stephenson, D. S. King, M. F. Goodman, J. Stone. J. Chem. Phys., 70, 4496 (1979).

236. J. L. Lyman. J. Chem. Phys., 67, 1868 (1977).

237. T. F. Deutsch, S. R. J. Brueck. J. Chem. Phys., 70, 2063 (1979).

238. D. S. Frankel, Jr. J. Chem. Phys., 65, 1696 (1976).

239. H. S. Kwok, E. Yablonovitch. Phys. Rev. Lett., 41, 745 (1978).

240. V. N. Bagratashvili, Yu. G. Vainer, V. S. Doljikov, S. F. Koliakov, A. A. Makarov, L. P. Malyavkin, E. A. Ryabov, E. G. Sil'kis, V. D. Titov. Pis'ma Zh. Eksp. Teor. Fiz. (Russian) 30, 502 (1979) [Sov. Phys. JETP Lett., 30, 471 (1979)].

241. V. N. Bagratashvili, Yu. G. Vainer, V. S. Doljikov, S. F. Koliakov, V. S. Letokhov, A. A. Makarov, L. P. Malyavkin, E. A. Ryabov, E. G. Sil'kis, V. D. Titov. $Z h$. Eksp. Teor. Fiz. (Russian) 80, 1008 (1981) [Sov. Phys. JETP, 53, 512 (1981)].

242. G. M. Zaslawsky, B. V. Chirikov. Uspekhi Fiz. Nauk (Russian), 105, 3 (1971) [Sov. Phys. Uspekhi. 24, 549 (1972)].

243. N. R. Isenor, V. Merchant, R. S. Hallsworth, M. C. Richardson. Can. J. Phys. 51, 1281 (1979).

244. R. V. Ambartzumian, N. V. Chekalin, V. S. Doljikov, V. S. Letokhov, E. A. Ryabov. Chem. Phys. Lett., 25, 515 (1974).

245. R. V. Ambartzumian, Yu. A. Gorokhov, V. S. Letokhov, G. N. Makarov, A. A. Puretzky. Zh. Eksp. Teor. Fiz. (Russian) 71, 440 (1976) [Sov. Phys. JETP, 44, 231 (1976)].

246. P. Kolodner, C. Winterfeld, E. Yablonovitch. Optics Comm., 20, 119 (1977).

247. R. V. Ambartzumian, Yu. A. Gorokhov, V. S. Letokhov, G. N. Makarov, A. A. Puretzky. Pis'ma Zh. Eksp. Teor. Fiz. (Russian) 22, 374 (1975) [Sov. Phys. JETP Lett. 22, 177 (1975)].

248. V. M. Akulin, S. S. Alimpiev, N. V: Karlov, L. A. Shelepin. Zh. Eksp. Teor. Fiz. (Russian) 69, 836 (1975) [Sov. Phys. JETP, 42, 427 (1975)].

249. D. M. Larsen, N. Bloembergen. Optics Comm., 17, 254 (1976).

250. D. M. Larsen. Optics Comm., 19, 404 (1976).

251. M. C. Gower, K. W. Billman. Optics Comm., 20, 133 (1977).

252. M. Rothschild, W. S. Tsay, D. O. Ham. Optics Comm., 24, 237 (1978).

253. M. J. Coggiola, P. A. Schulz. Y. T. Lee, Y. R. Shen. Phys. Rev. Lett., 38, 17 (1977).

254. Aa. S. Sudbo, P. A. Schulz, E. R. Grant, Y. R. Shen, Y. T. Lee. J. Chem. Phys., 68, 1306 (1978).

255. G. Folcher, W. Braun. J. Photochem., 8, 341 (1978).

256. J. W. Hadgens. J. Chem. Phys., 68, 777 (1978).

257. D. S. King, J. C. Stephenson. Chem. Phys. Lett., 51, 48 (1977).

258. V. N. Bagratashvili, V. N. Burimov, M. V. Kuzmin, V. S. Letokhov, A. P. Sviridov. Laser Chemistry, 1, (1982).

259. A. A. Makarov, G. N. Makarov, A. A. Puretzky, V. V. Tyakht. Appl. Phys. 23, 391 (1980).

260. Z. Karny, A. Gupta, R. N. Zare, S. T. Lin, J. Nieman, A. M. Ronn. Chem. Phys. 37, 15 (1979).

261. V. N. Bagratashvili, V. N. Burimov, D. E. Deev, Yu. A. Kudriavtzev, M. V. Kuzmin, V. S. Letokhov, A. P. Sviridov. Pis'ma Zh. Eksp. Teor. Fiz. (Russian), 35, 155 (1982). 